\title{
Notes on the Leonard System Classification
}

\author{
Paul Terwilliger ${ }^{1}$ (D)
}

Received: 16 January 2020 / Accepted: 14 June 2021 / Published online: 9 July 2021

(C) The Author(s) 2021

\begin{abstract}
Around 2001 we classified the Leonard systems up to isomorphism. The proof was lengthy and involved considerable computation. In this paper we give a proof that is shorter and involves minimal computation. We also give a comprehensive description of the intersection numbers of a Leonard system.
\end{abstract}

Keywords Association scheme $\cdot$ Leonard pair $\cdot$ Leonard system $\cdot q$-Racah polynomial

Mathematics Subject Classification 05E30 - 15A21

\section{Introduction}

In the area of Algebraic Combinatorics there is an object called a commutative association scheme $[2,13]$. This is a combinatorial generalization of a finite group, that retains enough of the group structure so that one can still speak of the character table. During the decade 1970-1980 it was realized by E. Bannai, P. Delsarte, D. Higman and others that commutative association schemes help to unify many aspects of group theory, coding theory, and design theory. An early work in this direction was the 1973 thesis of Delsarte [5]. This thesis helped to motivate the work of Bannai [2, p. i], who taught a series of graduate courses on commutative association schemes during September 1978-December 1982 at the Ohio State University. The lecture notes from those courses, along with more recent developments, became a book coauthored with T. Ito [2]. The book had a large impact; it is currently cited 698 times according to MathSciNet.

Paul Terwilliger

terwilli@math.wisc.edu

1 Department of Mathematics, University of Wisconsin, 480 Lincoln Drive, Madison, WI 53706-1388, USA 
In the Introduction to [2], Bannai and Ito describe the goals of their book. One goal was to summarize what is known about commutative association schemes up to that time. Another goal was to focus the reader's attention on two remarkable types of schemes, said to be $P$-polynomial and $Q$-polynomial. A $P$-polynomial scheme is essentially the same thing as a distance-regular graph, and can be viewed as a finite analog of a 2-point homogeneous space [25]. Similarly, a $Q$-polynomial scheme is a finite analog of a rank 1 symmetric space [25]. By a theorem of H. C. Wang [25], a compact Riemannian manifold is 2-point homogeneous if and only if it is rank 1 symmetric. This result was extended to the noncompact case by J. Tits [24] and S. Helgason [8]. Motivated by all this, Bannai and Ito conjectured that a primitive association scheme is $P$-polynomial if and only if it is $Q$-polynomial, provided that the diameter is sufficiently large [2, p. 312]. They also proposed the classification of schemes that are both $P$-polynomial and $Q$-polynomial [2, p. xiii].

Progress on the proposed classification was made while the book was still in preparation. A $P$-polynomial scheme gets its name from the fact that there exists a sequence of orthogonal polynomials $\left\{u_{i}\right\}_{i=0}^{d}$ such that $u_{i}(A)=A_{i} / k_{i}$ for $0 \leq i \leq d$, where $d$ is the diameter of the scheme, $A_{i}$ is the $i$ th associate matrix, $A=A_{1}$, and $k_{i}$ is the $i$ th valency [2, pp. 190, 261]. Similarly, for a $Q$-polynomial scheme there exists a sequence of orthogonal polynomials $\left\{u_{i}^{*}\right\}_{i=0}^{d}$ such that $u_{i}^{*}\left(A^{*}\right)=A_{i}^{*} / k_{i}^{*}$ for $0 \leq i \leq d$, where $A_{i}^{*}$ is the $i$ th dual associate matrix, $A^{*}=A_{1}^{*}$, and $k_{i}^{*}$ is the $i$ th dual valency [2, pp. 193, 261], [15, p. 384]. For schemes that are $P$-polynomial and $Q$ polynomial, we have $u_{i}\left(\theta_{j}\right)=u_{j}^{*}\left(\theta_{i}^{*}\right)$ for $0 \leq i, j \leq d$, where $\left\{\theta_{i}\right\}_{i=0}^{d}$ (resp. $\left.\left\{\theta_{i}^{*}\right\}_{i=0}^{d}\right)$ are the eigenvalues of $A$ (resp. $A^{*}$ ) [2, p. 262]. These equations are known as Delsarte duality [12] or Askey-Wilson duality [20, p. 261]. This duality can be defined for $d=\infty$, but throughout this paper we assume that $d$ is finite.

Askey-Wilson duality comes up naturally in the area of special functions and orthogonal polynomials. In this area the classical orthogonal polynomials are often described using a partially-ordered set called the Askey-tableau. The vertices in the poset represent the various families of classical orthogonal polynomials, and the covering relation describes what happens when a limit is taken. See [11] for an early version of the tableau, and [10, pp. 183, 413] for a more recent version. One branch of the tableau, sometimes called the terminating branch, contains the polynomials that are orthogonal with respect to a measure that is nonzero at finitely many arguments. At the top of this terminating branch sit the $q$-Racah polynomials, introduced in 1979 by R. Askey and J. Wilson [1]. The rest of the terminating branch consists of the $q$-Hahn, dual $q$-Hahn, $q$-Krawtchouk, dual $q$-Krawtchouk, quantum $q$-Krawtchouk, affine $q$-Krawtchouk, Racah, Hahn, dual-Hahn, and Krawtchouk polynomials. The above-named polynomials are defined using hypergeometric series or basic hypergeometric series, and it is transparent from the definition that they satisfy Askey-Wilson duality.

Back at Ohio State, there was a graduate student attending Bannai's classes by the name of Douglas Leonard. With Askey's encouragement, Leonard showed in [12] that the $q$-Racah polynomials give the most general orthogonal polynomial system that satisfies Askey-Wilson duality, under the assumption that $d \geq 9$. In [2, Theorem 5.1] Bannai and Ito give a version of Leonard's theorem that removes the 
assumption on $d$ and explicitly describes all the limiting cases that show up. This version gives a complete classification of the orthogonal polynomial systems that satisfy Askey-Wilson duality. It shows that the orthogonal polynomial systems that satisfy Askey-Wilson duality are from the terminating branch of the Askey-tableau, except for one family with $q=-1$ now called the Bannai-Ito polynomials [2, p. 271], [21, Example 5.14]. In our view, the terminating branch of the Askeytableau should include the Bannai-Ito polynomials. Adopting this view, for the rest of this paper we include the Bannai-Ito polynomials in the terminating branch of the Askey-tableau.

The Leonard theorem [2, Theorem 5.1] is notoriously complicated; the statement alone takes 11 pages. In an effort to simplify and clarify the theorem, the present author introduced the notion of a Leonard pair [18, Definition 1.1] and Leonard system [18, Definition 1.4]. Roughly speaking, a Leonard pair consists of two diagonalizable linear transformations on a finite-dimensional vector space, each acting on the eigenspaces of the other one in an irreducible tridiagonal fashion; see Definition 2.1 below. A Leonard system is essentially a Leonard pair, together with appropriate orderings of their eigenspaces; see Definition 2.3 below. In [18, Theorem 1.9] the Leonard systems are classified up to isomorphism. This classification is related to Leonard's theorem as follows. In [18, Appendix A] and [20, Sect. 19], a bijection is given between the isomorphism classes of Leonard systems over $\mathbb{R}$, and the orthogonal polynomial systems that satisfy Askey-Wilson duality. Given the bijection, the classification [18, Theorem 1.9] becomes a 'linearalgebraic version' of Leonard's theorem. This version is conceptually simple and quite elegant in our view. In [23] we start with the Leonard pair axiom and derive, in a uniform and attractive manner, the polynomials in the terminating branch of the Askey-tableau, along with their properties such as the 3-term recurrence, difference equation, Askey-Wilson duality, and orthogonality.

We comment on how the theory of Leonard pairs and Leonard systems depends on the choice of ground field. The classification [18, Theorem 1.9] shows that the ground field does not matter in a substantial way, unless it has characteristic 2 . In this case, the theory admits an additional family of polynomials called the orphans. The orphans have diameter $d=3$ only; they are described in [21, Example 5.15] and Example 20.13 below.

The book [2] appeared in 1984 and the paper [18] appeared in 2001. It is natural to ask what happened in between. The concept of a Leonard pair over $\mathbb{R}$ appears in [14, Definitions 1.1, 1.2], where it is called a thin Leonard pair. Also appearing in [14] is the correspondence between Leonard pairs over $\mathbb{R}$ and the orthogonal polynomial systems that satisfy Askey-Wilson duality. In addition [14, Definition 3.1] describes an algebra called the Leonard algebra, now known as the AskeyWilson algebra. The paper [14] was submitted but never published. In [26] A. Zhedanov introduced the Askey-Wilson algebra. This algebra has a presentation involving two generators $K_{1}, K_{2}$ that satisfy a pair of quadratic relations. In [6], Granovskii, Lutzenko, and Zhedanov consider a finite-dimensional irreducible module for the Askey-Wilson algebra, on which each of $K_{1}, K_{2}$ are diagonalizable. Under some minor assumptions, they show that each of $K_{1}, K_{2}$ acts in an irreducible tridiagonal fashion on an eigenbasis for the other one. In hindsight, it is fair to say 
that they constructed an example of a Leonard pair, although they did not define a Leonard pair as an independent concept. The paper [15, Theorem 2.1] contains a version of the Leonard pair concept that is close to [18, Definition 1.1].

Turning to the present paper, we obtain two main results: (i) an improved proof for the classification of Leonard systems; (ii) a comprehensive description of the intersection numbers of a Leonard system.

We now describe our main results in detail. In [18, Theorem 1.9] the Leonard systems are classified up to isomorphism, and the given proof is completely correct as far as we know. However the proof is longer than necessary. In the roughly two decades since the paper was published, we have discovered some 'shortcuts' that simplify the proof and avoid certain tedious calculations. The shortcuts are summarized as follows.

- In [18, Sect. 3] we established the split canonical form for a Leonard system. In the present paper we make use of the fact that the split canonical form still exists under weaker assumptions; these are described in Proposition 7.6 below.

- The concept of a normalizing idempotent was introduced by Edward Hanson in [7, Sect. 6]. In the present paper we use this concept to simplify numerous arguments; see Sects. 6, 7, 17 below.

- In [18, Theorem 4.8] we explicitly gave the matrix entries for a certain matrix representation of the primitive idempotents for a Leonard system. The computation of these entries is tedious and takes up most of [18, Sect. 4]. In the present paper we replace all of this by a single identity (15) that is established in a few lines.

- In the present paper we use an antiautomorphism $\dagger$ to obtain the result Proposition 4.4, which is roughly summarized as follows: as we construct a Leonard system, if we construct three-fourths of it then the last fourth comes for free.

- In [18, Lemma 7.2] we used a slightly obscure method to establish the irreducibility of the underlying module for a Leonard system. In the present paper this lemma is avoided using the first bullet point above.

- We replace the slightly technical results [18, Lemmas $10.3-10.5$ ] by a more elementary result, Proposition 13.4 below.

- We replace most of [18, Sect. 11] by a single result Proposition 8.4 called the wrap-around result. The wrap-around result was discovered by $\mathrm{T}$. Ito and the present author during our effort to classify the tridiagonal pairs; it is the essential idea behind the proof of [9, Lemma 9.9].

- Using the improvements listed above, we replace the arguments in [18, Sects. 13, 14] with more efficient arguments in Sect. 17 below.

Some parts of the improved proof are unchanged from the original; we still use [18, Sect. 8, 9] and [18, Lemmas 10.2, 12.4]. These results are reproduced in the present paper in order to obtain a complete proof, all in one place. We believe that this complete proof is suitable for The Book if not this journal.

Concerning our second main result, we mentioned earlier that the Leonard systems correspond to the orthogonal polynomial sequences that satisfy Askey- 
Wilson duality. Unfortunately, it is a bit difficult to go back and forth between the two points of view, because from the polynomial perspective, the main parameters are the intersection numbers (or connection coefficients) that describe the 3-term recurrence, and from the Leonard system perspective, the main parameters are the first and second split sequence that make up part of the parameter array. There are some equations that relate the two types of parameters; see [20, Theorem 17.7] and Lemma 19.4 below. However the nonlinear nature of these equations makes them difficult to use. In order to mitigate the difficulty, we display many identities that involve the intersection numbers along with the first and second split sequence. Taken together, these identities should make it easier to work with the intersection numbers in the future. These identities can be found in Sect. 19. We also explicitly give the intersection numbers for every isomorphism class of Leonard system; these are contained in the Appendix.

The paper is organized as follows. Sects. 2, 3 contain preliminary comments and definitions. In Sect. 4 we describe the antiautomorphism $\dagger$ and use it to obtain Proposition 4.4. In Sect. 5 we describe some polynomials that will be used throughout the paper. In Sect. 6 we discuss the concept of a normalizing idempotent. In Sect. 7 we use normalizing idempotents to describe certain kinds of decompositions relevant to Leonard systems. Section 8 contains the wrap-around result. In Sect. 9 we recall the parameter array of a Leonard system. In Sect. 10 we state the Leonard system classification, which is Theorem 10.1. Sections 11, 12, 13 are about recurrent sequences. In Sect. 14 we define a polynomial in two variables that will be useful on several occasions later in the paper. Sections 15, 16 are about the tridiagonal relations. In Sect. 17 we complete the proof of Theorem 10.1. Section 18 contains two characterizations related to Leonard systems and parameter arrays. In Sect. 19 we give a comprehensive treatment of the intersection numbers of a Leonard system. These intersection numbers are listed in the Appendix.

\section{Preliminaries}

We now begin our formal argument. Shortly we will define a Leonard pair and Leonard system. Before we get into detail, we briefly review some notation and basic concepts. Let $\mathbb{F}$ denote a field. Every vector space and algebra discussed in this paper is understood to be over $\mathbb{F}$. Throughout the paper fix an integer $d \geq 0$. Let $\operatorname{Mat}_{d+1}(\mathbb{F})$ denote the algebra consisting of the $d+1$ by $d+1$ matrices that have all entries in $\mathbb{F}$. We index the rows and columns by $0,1, \ldots, d$. Throughout the paper $V$ denotes a vector space with dimension $d+1$. Let $\operatorname{End}(V)$ denote the algebra consisting of the $\mathbb{F}$-linear maps from $V$ to $V$. Next we recall how each basis $\left\{v_{i}\right\}_{i=0}^{d}$ of $V$ gives an algebra isomorphism $\operatorname{End}(V) \rightarrow \operatorname{Mat}_{d+1}(\mathbb{F})$. For $X \in \operatorname{End}(V)$ and $M \in \operatorname{Mat}_{d+1}(\mathbb{F})$, we say that $M$ represents $X$ with respect to $\left\{v_{i}\right\}_{i=0}^{d}$ whenever $X v_{j}=\sum_{i=0}^{d} M_{i j} v_{i}$ for $0 \leq j \leq d$. The isomorphism sends $X$ to the unique matrix in $\operatorname{Mat}_{d+1}(\mathbb{F})$ that represents $X$ with respect to $\left\{v_{i}\right\}_{i=0}^{d}$. A matrix $M \in \operatorname{Mat}_{d+1}(\mathbb{F})$ is called tridiagonal whenever each nonzero entry lies on either the diagonal, the subdiagonal, or the superdiagonal. Assume that $M$ is tridiagonal. Then $M$ is called 
irreducible whenever each entry on the subdiagonal is nonzero, and each entry on the superdiagonal is nonzero.

Definition 2.1 (See [18, Definition 1.1]). By a Leonard pair on $V$ we mean an ordered pair $A, A^{*}$ of elements in $\operatorname{End}(V)$ such that:

(i) there exists a basis for $V$ with respect to which the matrix representing $A$ is diagonal and the matrix representing $A^{*}$ is irreducible tridiagonal;

(ii) there exists a basis for $V$ with respect to which the matrix representing $A^{*}$ is diagonal and the matrix representing $A$ is irreducible tridiagonal.

The Leonard pair $A, A^{*}$ is said to be over $\mathbb{F}$ and have diameter $d$.

Note 2.2 According to a common notational convention, $A^{*}$ denotes the conjugatetranspose of $A$. We are not using this convention. In a Leonard pair $A, A^{*}$ the linear transformations $A$ and $A^{*}$ are arbitrary subject to (i), (ii) above.

When working with a Leonard pair, it is convenient to consider a closely related object called a Leonard system. Before defining a Leonard system, we recall a few concepts from linear algebra. An element $A \in \operatorname{End}(V)$ is said to be diagonalizable whenever $V$ is spanned by the eigenspaces of $A$. The element $A$ is called multiplicityfree whenever $A$ is diagonalizable and each eigenspace of $A$ has dimension one. Note that $A$ is multiplicity-free if and only if $A$ has $d+1$ mutually distinct eigenvalues in $\mathbb{F}$. Assume that $A$ is multiplicity-free, and let $\left\{V_{i}\right\}_{i=0}^{d}$ denote an ordering of the eigenspaces of $A$. For $0 \leq i \leq d$ let $\theta_{i}$ denote the eigenvalue of $A$ for $V_{i}$. For $0 \leq i \leq d$ define $E_{i} \in \operatorname{End}(V)$ such that $\left(E_{i}-I\right) V_{i}=0$ and $E_{i} V_{j}=0$ if $j \neq i$ $(0 \leq j \leq d)$. We call $E_{i}$ the primitive idempotent of $A$ for $V_{i}$ (or $\left.\theta_{i}\right)$. We have (i) $E_{i} E_{j}=\delta_{i, j} E_{i}(0 \leq i, j \leq d)$; (ii) $I=\sum_{i=0}^{d} E_{i}$; (iii) $A E_{i}=\theta_{i} E_{i}=E_{i} A(0 \leq i \leq d)$; (iv) $A=\sum_{i=0}^{d} \theta_{i} E_{i}$; (v) $V_{i}=E_{i} V(0 \leq i \leq d)$; (vi) $\operatorname{rank}\left(E_{i}\right)=1(0 \leq i \leq d)$, (vii) $\operatorname{tr}\left(E_{i}\right)=$ $1(0 \leq i \leq d)$, where tr means trace. Moreover

$$
E_{i}=\prod_{\substack{0 \leq j \leq d \\ j \neq i}} \frac{A-\theta_{j} I}{\theta_{i}-\theta_{j}} \quad(0 \leq i \leq d) .
$$

Let $\mathcal{D}$ denote the subalgebra of $\operatorname{End}(V)$ generated by $A$. The elements $\left\{A^{i}\right\}_{i=0}^{d}$ form a basis for $\mathcal{D}$, and $\prod_{i=0}^{d}\left(A-\theta_{i} I\right)=0$. Moreover $\left\{E_{i}\right\}_{i=0}^{d}$ form a basis for $\mathcal{D}$.

Definition 2.3 (See [18, Definition 1.4]). By a Leonard system on $V$, we mean a sequence

$$
\Phi=\left(A ;\left\{E_{i}\right\}_{i=0}^{d} ; A^{*} ;\left\{E_{i}^{*}\right\}_{i=0}^{d}\right)
$$

of elements in $\operatorname{End}(V)$ that satisfy (i) $-(\mathrm{v})$ below:

(i) each of $A, A^{*}$ is multiplicity-free;

(ii) $\left\{E_{i}\right\}_{i=0}^{d}$ is an ordering of the primitive idempotents of $A$;

(iii) $\left\{E_{i}^{*}\right\}_{i=0}^{d}$ is an ordering of the primitive idempotents of $A^{*}$; 


$$
\begin{aligned}
E_{i}^{*} A E_{j}^{*} & =\left\{\begin{array}{cc}
0, & \text { if }|i-j|>1 ; \\
\neq 0, & \text { if }|i-j|=1
\end{array}\right. \\
E_{i} A^{*} E_{j} & =\left\{\begin{array}{cc}
0, & \text { if }|i-j|>1 ; \\
\neq 0, & \text { if }|i-j|=1
\end{array} \quad(0 \leq i, j \leq d)\right.
\end{aligned}
$$

The Leonard system $\Phi$ is said to be over $\mathbb{F}$ and have diameter $d$.

Leonard pairs and Leonard systems are related as follows. Let $\left(A ;\left\{E_{i}\right\}_{i=0}^{d} ; A^{*} ;\left\{E_{i}^{*}\right\}_{i=0}^{d}\right)$ denote a Leonard system on $V$. Then $A, A^{*}$ is a Leonard pair on $V$. Conversely, let $A, A^{*}$ denote a Leonard pair on $V$. Then each of $A, A^{*}$ is multiplicity-free [18, Lemma 1.3]. Moreover there exists an ordering $\left\{E_{i}\right\}_{i=0}^{d}$ of the primitive idempotents of $A$, and there exists an ordering $\left\{E_{i}^{*}\right\}_{i=0}^{d}$ of the primitive idempotents of $A^{*}$, such that $\left(A ;\left\{E_{i}\right\}_{i=0}^{d} ; A^{*} ;\left\{E_{i}^{*}\right\}_{i=0}^{d}\right)$ is a Leonard system on $V$.

Next we recall the notion of isomorphism for Leonard pairs and Leonard systems.

Definition 2.4 Let $A, A^{*}$ denote a Leonard pair on $V$, and let $B, B^{*}$ denote a Leonard pair on a vector space $V^{\prime}$. By an isomorphism of Leonard pairs from $A, A^{*}$ to $B, B^{*}$ we mean a vector space isomorphism $\sigma: V \rightarrow V^{\prime}$ such that $B=\sigma A \sigma^{-1}$ and $B^{*}=\sigma A^{*} \sigma^{-1}$. The Leonard pairs $A, A^{*}$ and $B, B^{*}$ are isomorphic whenever there exists an isomorphism of Leonard pairs from $A, A^{*}$ to $B, B^{*}$.

Let $\sigma: V \rightarrow V^{\prime}$ denote an isomorphism of vector spaces. For $X \in \operatorname{End}(V)$ abbreviate $X^{\sigma}=\sigma X \sigma^{-1}$ and note that $X^{\sigma} \in \operatorname{End}\left(V^{\prime}\right)$. The map $\operatorname{End}(V) \rightarrow \operatorname{End}\left(V^{\prime}\right)$, $X \mapsto X^{\sigma}$ is an isomorphism of algebras. For a Leonard system $\Phi=$ $\left(A ;\left\{E_{i}\right\}_{i=0}^{d} ; A^{*} ;\left\{E_{i}^{*}\right\}_{i=0}^{d}\right)$ on $V$ the sequence

$$
\Phi^{\sigma}:=\left(A^{\sigma} ;\left\{E_{i}^{\sigma}\right\}_{i=0}^{d} ; A^{* \sigma} ;\left\{E_{i}^{* \sigma}\right\}_{i=0}^{d}\right)
$$

is a Leonard system on $V^{\prime}$.

Definition 2.5 Let $\Phi$ denote a Leonard system on $V$, and let $\Phi^{\prime}$ denote a Leonard system on a vector space $V^{\prime}$. By an isomorphism of Leonard systems from $\Phi$ to $\Phi^{\prime}$, we mean an isomorphism of vector spaces $\sigma: V \rightarrow V^{\prime}$ such that $\Phi^{\sigma}=\Phi^{\prime}$. The Leonard systems $\Phi$ and $\Phi^{\prime}$ are isomorphic whenever there exists an isomorphism of Leonard systems from $\Phi$ to $\Phi^{\prime}$.

In [18, Theorem 1.9] we classified the Leonard systems up to isomorphism. Our first main goal in the present paper is to give an improved proof of this classifiction. This goal will be accomplished in Sects. 3, 4, 5, 6, 7, 8, 9, 10, 11, 12, 13, 14, 15, 16, 17. The statement of the classification is given in Theorem 10.1. The proof of Theorem 10.1 will be completed in Sect. 17.

Recall the commutator notation $[r, s]=r s-s r$. 


\section{Pre Leonard Systems}

As we start our investigation of Leonard systems, it is helpful to consider a more general object called a pre Leonard system. This object is defined as follows.

Definition 3.1 By a pre Leonard system on $V$, we mean a sequence

$$
\Phi=\left(A ;\left\{E_{i}\right\}_{i=0}^{d} ; A^{*} ;\left\{E_{i}^{*}\right\}_{i=0}^{d}\right)
$$

of elements in $\operatorname{End}(V)$ that satisfy conditions (i-iii) in Definition 2.3.

The results in this section refer to the pre Leonard system $\Phi$ from (3).

Definition 3.2 For $0 \leq i \leq d$ let $\theta_{i}$ (resp. $\theta_{i}^{*}$ ) denote the eigenvalue of $A$ (resp. $A^{*}$ ) for $E_{i}$ (resp. $E_{i}^{*}$ ). We call $\left\{\theta_{i}\right\}_{i=0}^{d}$ (resp. $\left\{\theta_{i}^{*}\right\}_{i=0}^{d}$ ) the eigenvalue sequence (resp. dual eigenvalue sequence) of $\Phi$. Let $\mathcal{D}$ (resp. $\left.\mathcal{D}^{*}\right)$ denote the subalgebra of $\operatorname{End}(V)$ generated by $A$ (resp. $A^{*}$ ).

Definition 3.3 Define

$$
a_{i}=\operatorname{tr}\left(A E_{i}^{*}\right), \quad a_{i}^{*}=\operatorname{tr}\left(A^{*} E_{i}\right) \quad(0 \leq i \leq d) .
$$

We call $\left\{a_{i}\right\}_{i=0}^{d}$ (resp. $\left\{a_{i}^{*}\right\}_{i=0}^{d}$ ) the diagonal sequence (resp. dual diagonal sequence) of $\Phi$.

Lemma 3.4 We have

$$
\begin{aligned}
& \theta_{0}+\theta_{1}+\cdots+\theta_{d}=a_{0}+a_{1}+\cdots+a_{d}, \\
& \theta_{0}^{*}+\theta_{1}^{*}+\cdots+\theta_{d}^{*}=a_{0}^{*}+a_{1}^{*}+\cdots+a_{d}^{*} .
\end{aligned}
$$

Proof To obtain (4), observe that

$$
\sum_{i=0}^{d} \theta_{i}=\operatorname{tr}(A)=\operatorname{tr}\left(A \sum_{i=0}^{d} E_{i}^{*}\right)=\sum_{i=0}^{d} a_{i} .
$$

The proof of (5) is similar.

Lemma 3.5 For $0 \leq i \leq d$,

(i) $E_{i}^{*} A E_{i}^{*}=a_{i} E_{i}^{*}$;

(ii) $E_{i} A^{*} E_{i}=a_{i}^{*} E_{i}$.

\section{Proof}

(i) Abbreviate $\mathcal{A}=\operatorname{End}(V)$. Since $E_{i}^{*}$ has rank 1 , the vector space $E_{i}^{*} \mathcal{A} E_{i}^{*}$ is spanned by $E_{i}^{*}$. Therefore there exists $\alpha_{i} \in \mathbb{F}$ such that $E_{i}^{*} A E_{i}^{*}=\alpha_{i} E_{i}^{*}$. In this equation take the trace of each side and use Definition 3.3 along with $\operatorname{tr}(X Y)=\operatorname{tr}(Y X)$ to obtain $a_{i}=\alpha_{i}$.

(ii) Similar to the proof of (i) above. 
We have been discussing the pre Leonard system

$$
\Phi=\left(A ;\left\{E_{i}\right\}_{i=0}^{d} ; A^{*} ;\left\{E_{i}^{*}\right\}_{i=0}^{d}\right)
$$

on $V$. Each of the following is a pre Leonard system on $V$ :

$$
\begin{aligned}
& \Phi^{\Downarrow}:=\left(A ;\left\{E_{d-i}\right\}_{i=0}^{d} ; A^{*} ;\left\{E_{i}^{*}\right\}_{i=0}^{d}\right), \\
& \Phi^{\downarrow}:=\left(A ;\left\{E_{i}\right\}_{i=0}^{d} ; A^{*} ;\left\{E_{d-i}^{*}\right\}_{i=0}^{d}\right), \\
& \Phi^{*}:=\left(A^{*} ;\left\{E_{i}^{*}\right\}_{i=0}^{d} ; A ;\left\{E_{i}\right\}_{i=0}^{d}\right) .
\end{aligned}
$$

Proposition 3.6 With the above notation,

\begin{tabular}{lllll}
\hline pre LS & Eigenvalue seq. & Dual eigenvalue seq. & Diagonal seq. & Dual diagonal seq. \\
\hline$\Phi$ & $\left\{\theta_{i}\right\}_{i=0}^{d}$ & $\left\{\theta_{i}^{*}\right\}_{i=0}^{d}$ & $\left\{a_{i}\right\}_{i=0}^{d}$ & $\left\{a_{i}^{*}\right\}_{i=0}^{d}$ \\
$\Phi^{\Downarrow}$ & $\left\{\theta_{d-i}\right\}_{i=0}^{d}$ & $\left\{\theta_{i}^{*}\right\}_{i=0}^{d}$ & $\left\{a_{i}\right\}_{i=0}^{d}$ & $\left\{a_{d-i}^{*}\right\}_{i=0}^{d}$ \\
$\Phi^{\downarrow}$ & $\left\{\theta_{i}\right\}_{i=0}^{d}$ & $\left\{\theta_{d-i}^{*}\right\}_{i=0}^{d}$ & $\left\{a_{d-i}\right\}_{i=0}^{d}$ & $\left\{a_{i}^{*}\right\}_{i=0}^{d}$ \\
$\Phi^{*}$ & $\left\{\theta_{i}^{*}\right\}_{i=0}^{d}$ & $\left\{\theta_{i}\right\}_{i=0}^{d}$ & $\left\{a_{i}^{*}\right\}_{i=0}^{d}$ & $\left\{a_{i}\right\}_{i=0}^{d}$ \\
\hline
\end{tabular}

Proof Use Definitions 3.2, 3.3.

\section{The Antiautomorphism $\dagger$}

We continue to discuss the pre Leonard system $\Phi=\left(A ;\left\{E_{i}\right\}_{i=0}^{d} ; A^{*} ;\left\{E_{i}^{*}\right\}_{i=0}^{d}\right)$ from Definition 3.1.

Lemma 4.1 Assume that

$$
E_{i}^{*} A E_{j}^{*}=\left\{\begin{array}{cc}
0, & \text { if }|i-j|>1 ; \\
\neq 0, & \text { if }|i-j|=1
\end{array} \quad(0 \leq i, j \leq d) .\right.
$$

Then the elements

$$
A^{i} E_{0}^{*} A^{j} \quad 0 \leq i, j \leq d
$$

form a basis for the vector space $\operatorname{End}(V)$.

Proof For $0 \leq i \leq d$ pick $0 \neq v_{i} \in E_{i}^{*} V$. So $\left\{v_{i}\right\}_{i=0}^{d}$ is a basis for $V$. Without loss of generality, we may identify each $X \in \operatorname{End}(V)$ with the matrix in $\operatorname{Mat}_{d+1}(\mathbb{F})$ that represents $X$ with respect to $\left\{v_{i}\right\}_{i=0}^{d}$. From this point of view $A$ is irreducible 
tridiagonal and $E_{0}^{*}=\operatorname{diag}(1,0, \ldots, 0)$. Using these matrices one routinely checks that the elements (6) are linearly independent. There are $(d+1)^{2}$ elements listed in (6), and this is the dimension of $\operatorname{End}(V)$. Therefore the elements (6) form a basis for $\operatorname{End}(V)$.

Lemma 4.2 Under the assumption in Lemma 4.1, each of the following is a generating set for the algebra $\operatorname{End}(V)$ : (i) $A, E_{0}^{*}$; (ii) $A, A^{*}$.

\section{Proof}

(i) By Lemma 4.1.

(ii) By (i) above and since $E_{0}^{*}$ is a polynomial in $A^{*}$.

By an automorphism of $\operatorname{End}(V)$ we mean an algebra isomorphism $\operatorname{End}(V) \rightarrow \operatorname{End}(V)$. By an antiautomorphism of $\operatorname{End}(V)$ we mean a vector space isomorphism $\zeta: \operatorname{End}(V) \rightarrow \operatorname{End}(V)$ such that $(X Y)^{\zeta}=Y^{\zeta} X^{\zeta}$ for all $X, Y \in \operatorname{End}(V)$.

Lemma 4.3 Under the assumption in Lemma 4.1,

(i) there exists a unique antiautomorphism $\dagger$ of $\operatorname{End}(V)$ that fixes each of $A, A^{*}$;

(ii) $\dagger$ fixes everything in $\mathcal{D}$ and everything in $\mathcal{D}^{*}$;

(iii) $\dagger$ fixes each of $E_{i}, E_{i}^{*}$ for $0 \leq i \leq d$;

(iv) $\left(X^{\dagger}\right)^{\dagger}=X$ for all $X \in \operatorname{End}(V)$.

\section{Proof}

(i) First we show that $\dagger$ exists. For $0 \leq i \leq d$ pick $0 \neq v_{i} \in E_{i}^{*} V$. So $\left\{v_{i}\right\}_{i=0}^{d}$ is a basis for $V$. For $X \in \operatorname{End}(V)$ let $X^{\sharp} \in \operatorname{Mat}_{d+1}(\mathbb{F})$ represent $X$ with respect to $\left\{v_{i}\right\}_{i=0}^{d}$. The map $\sharp: \operatorname{End}(V) \rightarrow \operatorname{Mat}_{d+1}(\mathbb{F}), X \mapsto X^{\sharp}$ is an algebra isomorphism. Write $B=A^{\sharp}$ and $B^{*}=A^{* \sharp}$. The matrix $B$ is irreducible tridiagonal and $B^{*}=\operatorname{diag}\left(\theta_{0}^{*}, \theta_{1}^{*}, \ldots, \theta_{d}^{*}\right)$. Define a diagonal matrix $K \in \mathrm{Mat}_{d+1}(\mathbb{F})$ with diagonal entries

$$
K_{i i}=\frac{B_{01} B_{12} \cdots B_{i-1, i}}{B_{10} B_{21} \cdots B_{i, i-1}} \quad(0 \leq i \leq d) .
$$

The matrix $K$ is invertible and $K^{-1} B^{t} K=B$. Define a map $b: \operatorname{Mat}_{d+1}(\mathbb{F}) \rightarrow \operatorname{Mat}_{d+1}(\mathbb{F}), X \mapsto K^{-1} X^{t} K$. The map $b$ is an antiautomorphism of $\mathrm{Mat}_{d+1}(\mathbb{F})$ that fixes each of $B, B^{*}$. The composition

$$
\dagger: \operatorname{End}(V) \underset{\sharp}{\longrightarrow} \operatorname{Mat}_{d+1}(\mathbb{F}) \underset{b}{\longrightarrow} \operatorname{Mat}_{d+1}(\mathbb{F}) \underset{\sharp-1}{\longrightarrow} \operatorname{End}(V)
$$

is an antiautomorphism of $\operatorname{End}(V)$ that fixes each of $A, A^{*}$. We have shown that $\dagger$ exists. Next we show that $\dagger$ is unique. Let $\zeta$ denote an antiautomorphism of $\operatorname{End}(V)$ that fixes each of $A, A^{*}$. Then the composition $\dagger \circ \zeta^{-1}$ is an automorphism of $\operatorname{End}(V)$ that fixes each of $A, A^{*}$. Now $\dagger \circ$ 
$\zeta^{-1}=1$ in view of Lemma 4.2(ii). Therefore $\dagger=\zeta$. We have shown that $\dagger$

(ii) Since $A$ (resp. $A^{*}$ ) generates $\mathcal{D}$ (resp. $\mathcal{D}^{*}$ ).

(iii) Since $E_{i} \in \mathcal{D}$ and $E_{i}^{*} \in \mathcal{D}^{*}$ for $0 \leq i \leq d$.

(iv) The composition $\dagger \circ \dagger$ is an automorphism of $\operatorname{End}(V)$ that fixes each of $A, A^{*}$. Now $\dagger \circ \dagger=1$ in view of Lemma 4.2(ii).

Proposition 4.4 Consider the following four conditions:

(i) $\quad E_{i}^{*} A E_{j}^{*}=\left\{\begin{array}{cc}0, & \text { if } i-j>1 ; \\ \neq 0, & \text { if } i-j=1\end{array} \quad(0 \leq i, j \leq d)\right.$;

(ii) $\quad E_{i}^{*} A E_{j}^{*}=\left\{\begin{array}{cc}0, & \text { if } j-i>1 ; \\ \neq 0, & \text { if } j-i=1\end{array} \quad(0 \leq i, j \leq d)\right.$;

(iii) $\quad E_{i} A^{*} E_{j}=\left\{\begin{array}{cc}0, & \text { if } i-j>1 ; \\ \neq 0, & \text { if } i-j=1\end{array} \quad(0 \leq i, j \leq d)\right.$;

(iv) $\quad E_{i} A^{*} E_{j}=\left\{\begin{array}{cc}0, & \text { if } j-i>1 ; \\ \neq 0, & \text { if } j-i=1\end{array} \quad(0 \leq i, j \leq d)\right.$.

Assume at least three of (i)-(iv) hold. Then each of (i)-(iv) holds; in other words the pre Leonard system $\Phi$ is a Leonard system.

Proof Interchanging $A, A^{*}$ if necessary, we may assume without loss of generality that (i), (ii) hold. Now the assumption of Lemma 4.1 holds, so Lemma 4.3 applies. Consider the map $\dagger$ from Lemma 4.3. For $0 \leq i, j \leq d$ we have

$$
\left(E_{i} A^{*} E_{j}\right)^{\dagger}=E_{j} A^{*} E_{i} \text {. }
$$

Therefore $E_{i} A^{*} E_{j}=0$ if and only if $E_{j} A^{*} E_{i}=0$. Consequently (iii) holds if and only if (iv) holds. The result follows.

\section{The Polynomials $\tau_{i}, \eta_{i}, \tau_{i}^{*}, \eta_{i}^{*}$}

We continue to discuss the pre Leonard system $\Phi=\left(A ;\left\{E_{i}\right\}_{i=0}^{d} ; A^{*} ;\left\{E_{i}^{*}\right\}_{i=0}^{d}\right)$ from Definition 3.1.

Let $\lambda$ denote an indeterminate. Let $\mathbb{F}[\lambda]$ denote the algebra consisting of the polynomials in $\lambda$ that have all coefficients in $\mathbb{F}$.

Definition 5.1 For $0 \leq i \leq d$ define $\tau_{i}, \eta_{i}, \tau_{i}^{*}, \eta_{i}^{*} \in \mathbb{F}[\lambda]$ by

$$
\begin{array}{ll}
\tau_{i}=\left(\lambda-\theta_{0}\right)\left(\lambda-\theta_{1}\right) \cdots\left(\lambda-\theta_{i-1}\right), & \eta_{i}=\left(\lambda-\theta_{d}\right)\left(\lambda-\theta_{d-1}\right) \cdots\left(\lambda-\theta_{d-i+1}\right), \\
\tau_{i}^{*}=\left(\lambda-\theta_{0}^{*}\right)\left(\lambda-\theta_{1}^{*}\right) \cdots\left(\lambda-\theta_{i-1}^{*}\right), & \eta_{i}^{*}=\left(\lambda-\theta_{d}^{*}\right)\left(\lambda-\theta_{d-1}^{*}\right) \cdots\left(\lambda-\theta_{d-i+1}^{*}\right) .
\end{array}
$$

Each of $\tau_{i}, \eta_{i}, \tau_{i}^{*}, \eta_{i}^{*}$ is monic with degree $i$. 
We mention some results about $\left\{\tau_{i}\right\}_{i=0}^{d}$ and $\left\{\eta_{i}\right\}_{i=0}^{d}$; similar results apply to $\left\{\tau_{i}^{*}\right\}_{i=0}^{d}$ and $\left\{\eta_{i}^{*}\right\}_{i=0}^{d}$.

Lemma 5.2 The vectors $\left\{\tau_{i}(A)\right\}_{i=0}^{d}$ form a basis for $\mathcal{D}$. Moreover the vectors $\left\{\eta_{i}(A)\right\}_{i=0}^{d}$ form a basis for $\mathcal{D}$.

Proof Since $\left\{A^{i}\right\}_{i=0}^{d}$ is a basis for $\mathcal{D}$, and $\tau_{i}, \eta_{i}$ have degree $i$ for $0 \leq i \leq d$.

Lemma 5.3 For $0 \leq i \leq d$,

(i) $\tau_{i}(A)=\sum_{h=i}^{d} \tau_{i}\left(\theta_{h}\right) E_{h}$;

(ii) $\eta_{i}(A)=\sum_{h=0}^{d-i} \eta_{i}\left(\theta_{h}\right) E_{h}$.

Proof

(i) We have $A=\sum_{h=0}^{d} \theta_{h} E_{h}$, so $\tau_{i}(A)=\sum_{h=0}^{d} \tau_{i}\left(\theta_{h}\right) E_{h}$. However $\tau_{i}\left(\theta_{h}\right)=0$ for $0 \leq h \leq i-1$, so $\tau_{i}(A)=\sum_{h=i}^{d} \tau_{i}\left(\theta_{h}\right) E_{h}$.

(ii) Apply (i) above to $\Phi^{\Downarrow}$.

Lemma 5.4 For $0 \leq i \leq d$,

(i) the elements $\left\{\tau_{j}(A)\right\}_{j=0}^{i}$ and $\left\{A^{j}\right\}_{j=0}^{i}$ have the same span;

(ii) the elements $\left\{\tau_{j}(A)\right\}_{j=i}^{d}$ and $\left\{E_{j}\right\}_{j=i}^{d}$ have the same span.

\section{Proof}

(i) The polynomial $\tau_{j}$ has degree $j$ for $0 \leq j \leq d$.

(ii) By Lemma 5.2 it suffices to show that the span of $\left\{\tau_{j}(A)\right\}_{j=i}^{d}$ is contained in the span of $\left\{E_{j}\right\}_{j=i}^{d}$. But this follows from Lemma 5.3(i).

Lemma 5.5 For $0 \leq i \leq d$,

(i) the elements $\left\{\eta_{j}(A)\right\}_{j=0}^{i}$ and $\left\{A^{j}\right\}_{j=0}^{i}$ have the same span;

(ii) the elements $\left\{\eta_{j}(A)\right\}_{j=i}^{d}$ and $\left\{E_{j}\right\}_{j=0}^{d-i}$ have the same span.

Proof Apply Lemma 5.4 to $\Phi^{\Downarrow}$.

\section{Normalizing Idempotents}

We continue to discuss the pre Leonard system $\Phi=\left(A ;\left\{E_{i}\right\}_{i=0}^{d} ; A^{*} ;\left\{E_{i}^{*}\right\}_{i=0}^{d}\right)$ from Definition 3.1. 
Next we explain what it means for $E_{0}^{*}$ to be normalizing. This concept was introduced in [7, Sect. 6], although our point of view is different.

Definition 6.1 The primitive idempotent $E_{0}^{*}$ is called normalizing whenever

$$
E_{i} E_{0}^{*} \neq 0 \quad(0 \leq i \leq d) .
$$

In the next two lemmas we give some necessary and sufficient conditions for $E_{0}^{*}$ to be normalizing. The proofs are routine, and omitted.

Lemma 6.2 The following (i)-(iv) are equivalent:

(i) $E_{0}^{*}$ is normalizing;

(ii) $\mathcal{D} E_{0}^{*}$ has dimension $d+1$;

(iii) the elements $\left\{A^{i} E_{0}^{*}\right\}_{i=0}^{d}$ are linearly independent;

(iv) for $X \in \mathcal{D}, X E_{0}^{*}=0$ implies $X=0$.

Lemma 6.3 The following (i)-(iv) are equivalent:

(i) $E_{0}^{*}$ is normalizing;

(ii) $E_{i} V=E_{i} E_{0}^{*} V$ for $0 \leq i \leq d$;

(iii) $\mathcal{D} E_{0}^{*} V=V$;

(iv) for $0 \neq \xi \in E_{0}^{*} V$ the map $\mathcal{D} \rightarrow V, X \mapsto X \xi$ is a bijection.

Proposition 6.4 Assume that $E_{0}^{*}$ is normalizing. Then for $X \in \operatorname{End}(V)$ and $0 \leq i \leq d$ the following are equivalent:

(i) $X E_{i}=0$

(ii) $X E_{i} E_{0}^{*}=0$.

Proof Using Lemma 6.3(ii),

$$
X E_{i}=0 \quad \Leftrightarrow \quad X E_{i} V=0 \quad \Leftrightarrow \quad X E_{i} E_{0}^{*} V=0 \quad \Leftrightarrow \quad X E_{i} E_{0}^{*}=0 .
$$

\section{Normalizing Idempotents and Decompositions}

We continue to discuss the pre Leonard system $\Phi=\left(A ;\left\{E_{i}\right\}_{i=0}^{d} ; A^{*} ;\left\{E_{i}^{*}\right\}_{i=0}^{d}\right)$ from Definition 3.1.

Definition 7.1 By a decomposition of $V$ we mean a sequence $\left\{V_{i}\right\}_{i=0}^{d}$ of onedimensional subspaces of $V$ such that the sum $V=\sum_{i=0}^{d} V_{i}$ is direct.

Example 7.2 Each of the sequences 


$$
\left\{E_{i} V\right\}_{i=0}^{d}, \quad\left\{E_{i}^{*} V\right\}_{i=0}^{d}
$$

is a decomposition of $V$.

Example 7.3 Let $\left\{v_{i}\right\}_{i=0}^{d}$ denote a basis for $V$. For $0 \leq i \leq d$ let $V_{i}$ denote the span of $v_{i}$. Then $\left\{V_{i}\right\}_{i=0}^{d}$ is a decomposition of $V$, said to be induced by $\left\{v_{i}\right\}_{i=0}^{d}$.

Lemma 7.4 Assume that $E_{0}^{*}$ is normalizing, and define

$$
U_{i}=\tau_{i}(A) E_{0}^{*} V \quad(0 \leq i \leq d) .
$$

Then the following (i)-(v) hold:

(i) $\left\{U_{i}\right\}_{i=0}^{d}$ is a decomposition of $V$;

(ii) $\quad\left(A-\theta_{i} I\right) U_{i}=U_{i+1} \quad(0 \leq i \leq d-1)$;

(iii) $\quad\left(A-\theta_{d} I\right) U_{d}=0$;

(iv) $\quad U_{0}+U_{1}+\cdots+U_{i}=E_{0}^{*} V+A E_{0}^{*} V+\cdots+A^{i} E_{0}^{*} V \quad(0 \leq i \leq d)$;

(v) $\quad U_{i}+U_{i+1}+\cdots+U_{d}=E_{i} V+E_{i+1} V+\cdots+E_{d} V \quad(0 \leq i \leq d)$.

Proof

(i) By Lemma 6.3(iv) and since $\left\{\tau_{i}(A)\right\}_{i=0}^{d}$ is a basis for $\mathcal{D}$.

(ii) By Definition 5.1.

(iii) Since $0=\prod_{i=0}^{d}\left(A-\theta_{i} I\right)=\left(A-\theta_{d} I\right) \tau_{d}(A)$.

(iv) By Lemma 5.4(i) and Lemma 6.3(iv).

(iv) By Lemma 5.4(ii) and Lemma 6.3(iv).

Lemma 7.5 The following (i)-(iii) are equivalent:

(i) $\quad E_{i}^{*} A E_{j}^{*}=\left\{\begin{array}{cc}0, & \text { if } i-j>1 ; \\ \neq 0, & \text { if } i-j=1\end{array} \quad(0 \leq i, j \leq d)\right.$;

(ii) for $0 \leq i \leq d$ there exists $f_{i} \in \mathbb{F}[\lambda]$ such that $\operatorname{deg}\left(f_{i}\right)=i$ and $E_{i}^{*} V=f_{i}(A) E_{0}^{*} V$

(iii) for $0 \leq i \leq d$,

$$
E_{0}^{*} V+E_{1}^{*} V+\cdots+E_{i}^{*} V=E_{0}^{*} V+A E_{0}^{*} V+\cdots+A^{i} E_{0}^{*} V .
$$

Assume that (i)-(iii) hold. Then $E_{0}^{*}$ is normalizing.

Proof (i) $\Rightarrow\left(\right.$ ii) For $0 \leq i \leq d$ pick $0 \neq v_{i} \in E_{i}^{*} V$. So $\left\{v_{i}\right\}_{i=0}^{d}$ is a basis for $V$. Let $B \in \operatorname{Mat}_{d+1}(\mathbb{F})$ represent $A$ with respect to $\left\{v_{i}\right\}_{i=0}^{d}$. The entries of $B$ satisfy

$$
B_{i j}=\left\{\begin{array}{cc}
0, & \text { if } i-j>1 ; \\
\neq 0, & \text { if } i-j=1
\end{array} \quad(0 \leq i, j \leq d) .\right.
$$

Define polynomials $\left\{f_{i}\right\}_{i=0}^{d}$ in $\mathbb{E}[\lambda]$ by $f_{0}=1$ and 


$$
\lambda f_{j}=\sum_{i=0}^{j+1} B_{i j} f_{i} \quad(0 \leq j \leq d-1) .
$$

For $0 \leq i \leq d$ the polynomial $f_{i}$ has degree $i$. Also $v_{i}=f_{i}(A) v_{0}$, so $E_{i}^{*} V=f_{i}(A) E_{0}^{*} V$.

(ii) $\Rightarrow$ (iii) The polynomial $f_{j}$ has degree at most $i$ for $0 \leq j \leq i$, so

$$
E_{0}^{*} V+E_{1}^{*} V+\cdots+E_{i}^{*} V \subseteq E_{0}^{*} V+A E_{0}^{*} V+\cdots+A^{i} E_{0}^{*} V .
$$

In this inclusion, the left-hand side has dimension $i+1$ and the right-hand side has dimension at most $i+1$. Therefore the inclusion holds with equality.

(iii) $\Rightarrow\left(\right.$ i) For $0 \leq i \leq d$ let $V_{i}$ denote the common value in (7). Observe that

$$
E_{i}^{*} V_{j}=\left\{\begin{array}{cc}
0, & \text { if } i>j ; \\
\neq 0, & \text { if } i \leq j
\end{array} \quad(0 \leq i, j \leq d) .\right.
$$

Also observe that

$$
V_{j+1}=V_{j}+A V_{j} \quad(0 \leq j \leq d-1) .
$$

Now for $0 \leq i, j \leq d$ we check the conditions in (i). First assume that $i-j>1$. Then

$$
E_{i}^{*} A E_{j}^{*} V \subseteq E_{i}^{*} A V_{j} \subseteq E_{i}^{*} V_{j+1}=0,
$$

so $E_{i}^{*} A E_{j}^{*}=0$. Next assume that $i-j=1$. To show that $E_{i}^{*} A E_{j}^{*} \neq 0$, we suppose $E_{i}^{*} A E_{j}^{*}=0$ and get a contradiction. For $0 \leq h \leq i-1$ we have $E_{i}^{*} A E_{h}^{*}=0$, so $E_{i}^{*} A E_{h}^{*} V=0$. Therefore $E_{i}^{*} A V_{i-1}=0$. We also have $E_{i}^{*} V_{i-1}=0$, so $E_{i}^{*} V_{i}=E_{i}^{*}\left(V_{i-1}+A V_{i-1}\right)=0$, for a contradiction. Therefore $E_{i}^{*} A E_{j}^{*} \neq 0$.

Assume that (i)-(iii) hold. Setting $i=d$ in (iii) we obtain $V=\mathcal{D} E_{0}^{*} V$. Consequently $E_{0}^{*}$ is normalizing by Lemma 6.3(i),(iii).

Proposition 7.6 The following (i)-(iii) are equivalent:

(i) Both

$$
\begin{gathered}
E_{i}^{*} A E_{j}^{*}=\left\{\begin{array}{cc}
0, & \text { if } i-j>1 ; \\
\neq 0, & \text { if } i-j=1
\end{array} \quad(0 \leq i, j \leq d),\right. \\
E_{i} A^{*} E_{j}=0 \quad \text { if } j-i>1 \quad(0 \leq i, j \leq d) .
\end{gathered}
$$

(ii) There exists a decomposition $\left\{U_{i}\right\}_{i=0}^{d}$ of $V$ such that

$$
\begin{aligned}
& \left(A-\theta_{i} I\right) U_{i}=U_{i+1} \quad(0 \leq i \leq d-1), \quad\left(A-\theta_{d} I\right) U_{d}=0, \\
& \left(A^{*}-\theta_{i}^{*} I\right) U_{i} \subseteq U_{i-1} \quad(1 \leq i \leq d), \quad\left(A^{*}-\theta_{0}^{*} I\right) U_{0}=0 .
\end{aligned}
$$

(iii) There exist scalars $\left\{\varphi_{i}\right\}_{i=1}^{d}$ in $\mathbb{F}$ and a basis for $V$ with respect to which 


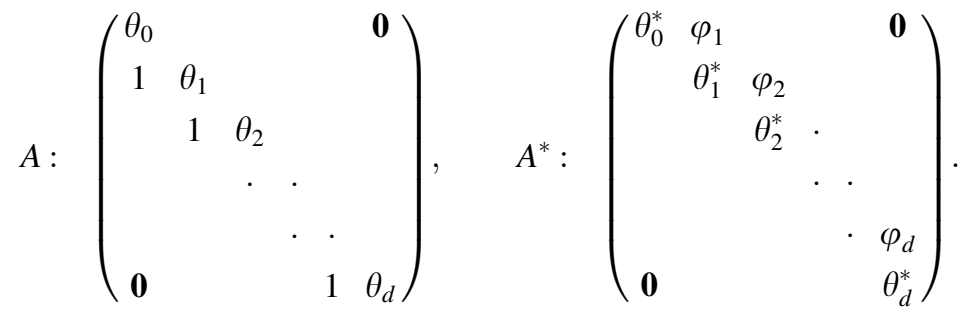

Assume that (i)-(iii) hold. Then $E_{0}^{*}$ is normalizing and $U_{i}=\tau_{i}(A) E_{0}^{*} V$ for $0 \leq i \leq d$. The basis for $V$ from (iii) is $\left\{\tau_{i}(A) \xi\right\}_{i=0}^{d}$, where $0 \neq \xi \in E_{0}^{*} V$. This basis induces the decomposition $\left\{U_{i}\right\}_{i=0}^{d}$. The sequence $\left\{\varphi_{i}\right\}_{i=1}^{d}$ is unique.

Proof (i) $\Rightarrow$ (ii) The element $E_{0}^{*}$ is normalizing by Lemma 7.5, so Lemma 7.4 applies. Consider the decomposition $\left\{U_{i}\right\}_{i=0}^{d}$ of $V$ from Lemma 7.4. This decomposition satisfies (10) by Lemma 7.4(ii),(iii). We now show (11). By Lemma 7.4(iv) and Lemma 7.5(iii),

$$
U_{0}+\cdots+U_{i}=E_{0}^{*} V+\cdots+E_{i}^{*} V \quad(0 \leq i \leq d) .
$$

For $0 \leq i \leq d$,

$$
\begin{aligned}
\left(A^{*}-\theta_{i}^{*} I\right) U_{i} & \subseteq\left(A^{*}-\theta_{i}^{*} I\right)\left(U_{0}+\cdots+U_{i}\right) \\
& =\left(A^{*}-\theta_{i}^{*} I\right)\left(E_{0}^{*} V+\cdots+E_{i}^{*} V\right) \\
& =E_{0}^{*} V+\cdots+E_{i-1}^{*} V \\
& =U_{0}+\cdots+U_{i-1} .
\end{aligned}
$$

Also, using Lemma 7.4(v) and (9),

$$
\begin{aligned}
\left(A^{*}-\theta_{i}^{*} I\right) U_{i} & \subseteq\left(A^{*}-\theta_{i}^{*} I\right)\left(U_{i}+\cdots+U_{d}\right) \\
& =\left(A^{*}-\theta_{i}^{*} I\right)\left(E_{i} V+\cdots+E_{d} V\right) \\
& \subseteq E_{i-1} V+\cdots+E_{d} V \\
& =U_{i-1}+\cdots+U_{d} .
\end{aligned}
$$

The above comments imply (11).

(ii) $\Rightarrow$ (i) From (11) we obtain

$$
U_{0}+\cdots+U_{i}=E_{0}^{*} V+\cdots+E_{i}^{*} V \quad(0 \leq i \leq d) .
$$

In particular $U_{0}=E_{0}^{*} V$. By this and (10) we obtain $U_{i}=\tau_{i}(A) E_{0}^{*} V$ for $0 \leq i \leq d$. Consequently $V=\mathcal{D} E_{0}^{*} V$, so $E_{0}^{*}$ is normalizing by Lemma 6.3(i),(iii). We show (8). Combining Lemma 7.4(iv) and (13) we obtain Lemma 7.5(iii). This gives Lemma 7.5(i), which is (8). Next we show (9). Let $i, j$ be given with $j-i>1$. Using Lemma 7.4(v) and (11), 


$$
\begin{aligned}
E_{i} A^{*} E_{j} V & \subseteq E_{i} A^{*}\left(E_{j} V+\cdots+E_{d} V\right) \\
& =E_{i} A^{*}\left(U_{j}+\cdots+U_{d}\right) \\
& \subseteq E_{i}\left(U_{j-1}+\cdots+U_{d}\right) \\
& =E_{i}\left(E_{j-1} V+\cdots+E_{d} V\right) \\
& =0 .
\end{aligned}
$$

Therefore $E_{i} A^{*} E_{j}=0$. We have shown (9).

(ii) $\Leftrightarrow$ (iii) Assertion (iii) is a reformulation of (ii) in terms of matrices.

Now assume that (i)-(iii) hold. We mentioned in the proof of (ii) $\Rightarrow$ (i) that $E_{0}^{*}$ is normalizing and $U_{i}=\tau_{i}(A) E_{0}^{*} V$ for $0 \leq i \leq d$. Let $\left\{u_{i}\right\}_{i=0}^{d}$ denote the basis for $V$ from (iii), and define $\xi=u_{0}$. From the matrix representing $A^{*}$ in (12), we see that $\xi$ is an eigenvector for $A^{*}$ with eigenvalue $\theta_{0}^{*}$. So $\xi \in E_{0}^{*} V$. From the matrix representing $A$ in (12), we obtain $\left(A-\theta_{i} I\right) u_{i}=u_{i+1}$ for $0 \leq i \leq d-1$. Consequently $u_{i}=\tau_{i}(A) \xi$ for $0 \leq i \leq d$. For $0 \leq i \leq d$ we have $u_{i}=\tau_{i}(A) \xi \in \tau_{i}(A) E_{0}^{*} V=U_{i}$. So the basis $\left\{u_{i}\right\}_{i=0}^{d}$ induces the decomposition $\left\{U_{i}\right\}_{i=0}^{d}$. The sequence $\left\{\varphi_{i}\right\}_{i=1}^{d}$ is unique since the vector $\xi$ is unique up to multiplication by a nonzero scalar.

Lemma 7.7 Assume that the equivalent conditions (i)-(iii) hold in Proposition 7.6. Then for $1 \leq i \leq d$ the following (i)-(iii) are equivalent:

(i) $E_{i-1} A^{*} E_{i} \neq 0$;

(ii) $\quad\left(A^{*}-\theta_{i}^{*} I\right) U_{i}=U_{i-1}$;

(iii) $\varphi_{i} \neq 0$.

Proof (i) $\Rightarrow$ (ii) We assume that $\left(A^{*}-\theta_{i}^{*} I\right) U_{i} \neq U_{i-1}$ and get a contradiction. We have $\left(A^{*}-\theta_{i}^{*} I\right) U_{i}=0$ since $\left(A^{*}-\theta_{i}^{*} I\right) U_{i} \subseteq U_{i-1}$ and $U_{i-1}$ has dimension one. Using Lemma 7.4(v),

$$
\begin{aligned}
E_{i-1} A^{*} E_{i} V & \subseteq E_{i-1} A^{*}\left(E_{i} V+\cdots+E_{d} V\right) \\
& =E_{i-1} A^{*}\left(U_{i}+\cdots+U_{d}\right) \\
& \subseteq E_{i-1}\left(U_{i}+\cdots+U_{d}\right) \\
& =E_{i-1}\left(E_{i} V+\cdots+E_{d} V\right) \\
& =0
\end{aligned}
$$

Therefore $E_{i-1} A^{*} E_{i}=0$ for a contradiction. We have shown $\left(A^{*}-\theta_{i}^{*} I\right) U_{i}=U_{i-1}$.

(ii) $\Rightarrow$ (i) We assume that $E_{i-1} A^{*} E_{i}=0$ and get a contradiction. Using Lemma 7.4(v), 


$$
\begin{aligned}
U_{i-1} & =\left(A^{*}-\theta_{i}^{*} I\right) U_{i} \\
& \subseteq\left(A^{*}-\theta_{i}^{*} I\right)\left(U_{i}+\cdots+U_{d}\right) \\
& =\left(A^{*}-\theta_{i}^{*} I\right)\left(E_{i} V+\cdots+E_{d} V\right) \\
& \subseteq E_{i} V+\cdots+E_{d} V \\
& =U_{i}+\cdots+U_{d} .
\end{aligned}
$$

This contradicts the fact that $\left\{U_{i}\right\}_{i=0}^{d}$ is a decomposition. We have shown $E_{i-1} A^{*} E_{i} \neq 0$.

(ii) $\Leftrightarrow$ (iii) Use the matrix representation of $A^{*}$ from (12).

\section{A Result About Wrap-Around}

We continue to discuss the pre Leonard system $\Phi=\left(A ;\left\{E_{i}\right\}_{i=0}^{d} ; A^{*} ;\left\{E_{i}^{*}\right\}_{i=0}^{d}\right)$ from Definition 3.1.

Throughout this section we assume that $\Phi$ satisfies the equivalent conditions (iiii) in Proposition 7.6. We will obtain a useful result involving the scalars $\left\{\varphi_{i}\right\}_{i=1}^{d}$ from Proposition 7.6(iii); this result is sometimes called the wrap-around result.

Recall the parameters $\left\{a_{i}\right\}_{i=0}^{d},\left\{a_{i}^{*}\right\}_{i=0}^{d}$ from Definition 3.3. We next compute these parameters in terms of $\left\{\theta_{i}\right\}_{i=0}^{d},\left\{\theta_{i}^{*}\right\}_{i=0}^{d},\left\{\varphi_{i}\right\}_{i=1}^{d}$. First assume that $d=0$. Then $A=\theta_{0} I$ and $A^{*}=\theta_{0}^{*} I$, so $a_{0}=\theta_{0}$ and $a_{0}^{*}=\theta_{0}^{*}$.

Lemma 8.1 (See [18, Lemma 5.1]). For $d \geq 1$ we have

$$
\begin{aligned}
a_{0} & =\theta_{0}+\frac{\varphi_{1}}{\theta_{0}^{*}-\theta_{1}^{*}}, \\
a_{i} & =\theta_{i}+\frac{\varphi_{i}}{\theta_{i}^{*}-\theta_{i-1}^{*}}+\frac{\varphi_{i+1}}{\theta_{i}^{*}-\theta_{i+1}^{*}} \quad(1 \leq i \leq d-1), \\
a_{d} & =\theta_{d}+\frac{\varphi_{d}}{\theta_{d}^{*}-\theta_{d-1}^{*}}
\end{aligned}
$$

and

$$
\begin{aligned}
& a_{0}^{*}=\theta_{0}^{*}+\frac{\varphi_{1}}{\theta_{0}-\theta_{1}}, \\
& a_{i}^{*}=\theta_{i}^{*}+\frac{\varphi_{i}}{\theta_{i}-\theta_{i-1}}+\frac{\varphi_{i+1}}{\theta_{i}-\theta_{i+1}} \quad(1 \leq i \leq d-1), \\
& a_{d}^{*}=\theta_{d}^{*}+\frac{\varphi_{d}}{\theta_{d}-\theta_{d-1}} .
\end{aligned}
$$

Proof Concerning $\left\{a_{i}\right\}_{i=0}^{d}$, define 


$$
\begin{aligned}
& x_{0}=\theta_{0}+\frac{\varphi_{1}}{\theta_{0}^{*}-\theta_{1}^{*}}, \\
& x_{i}=\theta_{i}+\frac{\varphi_{i}}{\theta_{i}^{*}-\theta_{i-1}^{*}}+\frac{\varphi_{i+1}}{\theta_{i}^{*}-\theta_{i+1}^{*}} \quad(1 \leq i \leq d-1), \\
& x_{d}=\theta_{d}+\frac{\varphi_{d}}{\theta_{d}^{*}-\theta_{d-1}^{*}} .
\end{aligned}
$$

We show that $a_{i}=x_{i}$ for $0 \leq i \leq d$. Since $\left\{\theta_{i}^{*}\right\}_{i=0}^{d}$ are mutually distinct, it suffices to show that $0=\sum_{i=0}^{d}\left(x_{i}-a_{i}\right) \theta_{i}^{* r}$ for $0 \leq r \leq d$. Let $r$ be given. We compute the trace of $A A^{* r}$ in two ways. On one hand, $A^{*}=\sum_{i=0}^{d} \theta_{i}^{*} E_{i}^{*}$ so $A^{* r}=\sum_{i=0}^{d} \theta_{i}^{* r} E_{i}^{*}$. By this and Definition 3.3,

$$
\operatorname{tr}\left(A A^{* r}\right)=\sum_{i=0}^{d} a_{i} \theta_{i}^{* r} .
$$

On the other hand, consider the matrix representations of $A$ and $A^{*}$ from (12). Using these matrices we compute the trace of $A A^{* r}$ as the sum of the diagonal entries. A brief calculation yields

$$
\operatorname{tr}\left(A A^{* r}\right)=\sum_{i=0}^{d} \theta_{i} \theta_{i}^{* r}+\sum_{i=1}^{d} \varphi_{i} \frac{\theta_{i-1}^{* r}-\theta_{i}^{* r}}{\theta_{i-1}^{*}-\theta_{i}^{*}} .
$$

Comparing (14), (15) we get an equation that becomes $0=\sum_{i=0}^{d}\left(x_{i}-a_{i}\right) \theta_{i}^{* r}$ after rearranging the terms. We have shown that $a_{i}=x_{i}$ for $0 \leq i \leq d$. Our assertions concerning $\left\{a_{i}^{*}\right\}_{i=0}^{d}$ are similarly obtained, by computing in two ways the trace of $A^{*} A^{r}$ for $0 \leq r \leq d$.

Lemma 8.2 (See [18, Lemma 5.2]). For $1 \leq i \leq d$ the scalar $\varphi_{i}$ is equal to each of the following four expressions:

$$
\begin{array}{ll}
\left(\theta_{i}^{*}-\theta_{i-1}^{*}\right) \sum_{h=0}^{i-1}\left(\theta_{h}-a_{h}\right), & \left(\theta_{i-1}^{*}-\theta_{i}^{*}\right) \sum_{h=i}^{d}\left(\theta_{h}-a_{h}\right), \\
\left(\theta_{i}-\theta_{i-1}\right) \sum_{h=0}^{i-1}\left(\theta_{h}^{*}-a_{h}^{*}\right), & \left(\theta_{i-1}-\theta_{i}\right) \sum_{h=i}^{d}\left(\theta_{h}^{*}-a_{h}^{*}\right) .
\end{array}
$$

Proof Use Lemmas 3.4, 8.1.

Definition 8.3 Define

$$
\vartheta_{i}=\varphi_{i}-\left(\theta_{i}^{*}-\theta_{0}^{*}\right)\left(\theta_{i-1}-\theta_{d}\right) \quad(1 \leq i \leq d)
$$

and $\vartheta_{0}=0, \vartheta_{d+1}=0$.

Proposition 8.4 (wrap-around) Assume $d \geq 2$. Then 


$$
\sum_{i=0}^{d-2} E_{d} A^{*} E_{i} E_{0}^{*}\left(\theta_{i}-\theta_{d-1}\right)=E_{d} E_{0}^{*}\left(\vartheta_{1}-\vartheta_{d}\right)
$$

Proof In the equation $I=\sum_{i=0}^{d} E_{i}^{*}$, multiply each term on the right by $A E_{0}^{*}$. Simplify the result using $E_{0}^{*} A E_{0}^{*}=a_{0} E_{0}^{*}$ and $E_{i}^{*} A E_{0}^{*}=0(2 \leq i \leq d)$ to obtain

$$
A E_{0}^{*}=a_{0} E_{0}^{*}+E_{1}^{*} A E_{0}^{*} .
$$

In (16), multiply each term on the left by $A^{*}$ and simplify to get

$$
A^{*} A E_{0}^{*}=a_{0} \theta_{0}^{*} E_{0}^{*}+\theta_{1}^{*} E_{1}^{*} A E_{0}^{*} .
$$

In the equation $I=\sum_{i=0}^{d} E_{i}$, multiply each term on the left by $E_{d} A^{*}$. Simplify the result using $E_{d} A^{*} E_{d}=a_{d}^{*} E_{d}$ to obtain

$$
E_{d} A^{*}=a_{d}^{*} E_{d}+\sum_{i=0}^{d-1} E_{d} A^{*} E_{i}
$$

In (18), multiply each term on the right by $A$ and simplify to obtain

$$
E_{d} A^{*} A=a_{d}^{*} \theta_{d} E_{d}+\sum_{i=0}^{d-1} \theta_{i} E_{d} A^{*} E_{i} .
$$

We now compute $\theta_{1}^{*} E_{d}$ times (16) minus $E_{d}$ times (17) minus (18) times $\theta_{d-1} E_{0}^{*}$ plus (19) times $E_{0}^{*}$. The result is

$$
E_{d} E_{0}^{*}\left(\left(\theta_{0}^{*}-\theta_{1}^{*}\right) a_{0}+\left(\theta_{d-1}-\theta_{d}\right) a_{d}^{*}+\theta_{d} \theta_{1}^{*}-\theta_{d-1} \theta_{0}^{*}\right)=\sum_{i=0}^{d-2} E_{d} A^{*} E_{i} E_{0}^{*}\left(\theta_{i}-\theta_{d-1}\right) .
$$

In the above equation, consider the coefficient of $E_{d} E_{0}^{*}$. Evaluate this coefficient using

$$
\begin{array}{lrl}
a_{0}=\theta_{0}+\frac{\varphi_{1}}{\theta_{0}^{*}-\theta_{1}^{*}}, & \varphi_{1}=\vartheta_{1}+\left(\theta_{1}^{*}-\theta_{0}^{*}\right)\left(\theta_{0}-\theta_{d}\right), \\
a_{d}^{*}=\theta_{d}^{*}+\frac{\varphi_{d}}{\theta_{d}-\theta_{d-1}}, & \varphi_{d}=\vartheta_{d}+\left(\theta_{d}^{*}-\theta_{0}^{*}\right)\left(\theta_{d-1}-\theta_{d}\right)
\end{array}
$$

to find that this coefficient is $\vartheta_{1}-\vartheta_{d}$.

For the sake of completeness, we mention a second version of Proposition 8.4. We do not use this second version, so we will not dwell on the proof.

Lemma 8.5 Assume $d \geq 2$. Then 


$$
\sum_{i=2}^{d} E_{0}^{*} A E_{i}^{*} E_{d}\left(\theta_{1}^{*}-\theta_{i}^{*}\right)=E_{0}^{*} E_{d}\left(\vartheta_{1}-\vartheta_{d}\right) .
$$

Proof Similar to the proof of Proposition 8.4.

\section{The Parameter Array of a Leonard System}

In this section we consider a Leonard system $\Phi=\left(A ;\left\{E_{i}\right\}_{i=0}^{d} ; A^{*} ;\left\{E_{i}^{*}\right\}_{i=0}^{d}\right)$ on $V$. Note that $\Phi$ satisfies the equivalent conditions (i)-(iii) in Proposition 7.6.

Definition 9.1 By the first split sequence for $\Phi$ we mean the sequence $\left\{\varphi_{i}\right\}_{i=1}^{d}$ from Proposition 7.6(iii). Let $\left\{\phi_{i}\right\}_{i=1}^{d}$ denote the first split sequence for $\Phi^{\Downarrow}$. We call $\left\{\phi_{i}\right\}_{i=1}^{d}$ the second split sequence for $\Phi$.

By Lemma 7.7 and the construction, $\varphi_{i}$ and $\phi_{i}$ are nonzero for $1 \leq i \leq d$.

Lemma 9.2 There exists a basis for $V$ with respect to which

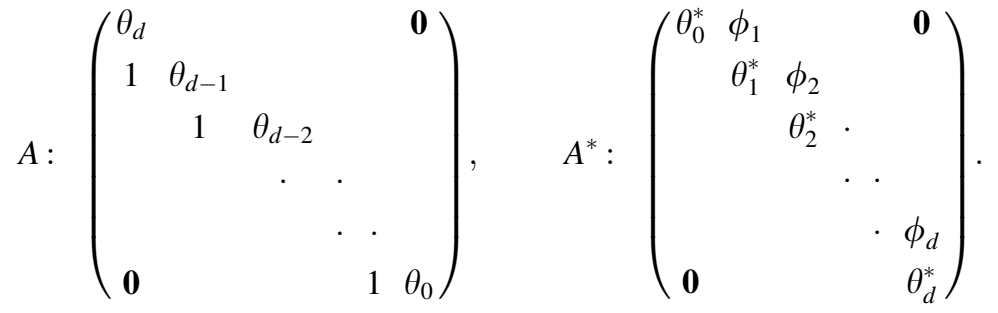

Proof Apply Proposition 7.6(iii) to $\Phi^{\Downarrow}$.

Lemma 9.3 For a Leonard system $\Phi^{\prime}$ over $\mathbb{F}$, the following are equivalent:

(i) $\Phi, \Phi^{\prime}$ are isomorphic;

(ii) $\Phi, \Phi^{\prime}$ have the same eigenvalue sequence, dual eigenvalue sequence, and first split sequence;

(iii) $\Phi, \Phi^{\prime}$ have the same eigenvalue sequence, dual eigenvalue sequence, and second split sequence.

Proof (i) $\Leftrightarrow$ (ii) By Proposition 7.6(iii).

(i) $\Leftrightarrow$ (iii) By Lemma 9.2.

In Lemma 8.1 we gave some formulas for $\left\{a_{i}\right\}_{i=0}^{d},\left\{a_{i}^{*}\right\}_{i=0}^{d}$ that involved $\left\{\varphi_{i}\right\}_{i=1}^{d}$. Next we give some similar formulas that involve $\left\{\phi_{i}\right\}_{i=1}^{d}$.

Lemma 9.4 For $d \geq 1$ we have 


$$
\begin{aligned}
& a_{0}=\theta_{d}+\frac{\phi_{1}}{\theta_{0}^{*}-\theta_{1}^{*}} \\
& a_{i}=\theta_{d-i}+\frac{\phi_{i}}{\theta_{i}^{*}-\theta_{i-1}^{*}}+\frac{\phi_{i+1}}{\theta_{i}^{*}-\theta_{i+1}^{*}} \quad(1 \leq i \leq d-1), \\
& a_{d}=\theta_{0}+\frac{\phi_{d}}{\theta_{d}^{*}-\theta_{d-1}^{*}}
\end{aligned}
$$

and

$$
\begin{aligned}
& a_{0}^{*}=\theta_{d}^{*}+\frac{\phi_{d}}{\theta_{0}-\theta_{1}}, \\
& a_{i}^{*}=\theta_{d-i}^{*}+\frac{\phi_{d-i+1}}{\theta_{i}-\theta_{i-1}}+\frac{\phi_{d-i}}{\theta_{i}-\theta_{i+1}} \quad(1 \leq i \leq d-1), \\
& a_{d}^{*}=\theta_{0}^{*}+\frac{\phi_{1}}{\theta_{d}-\theta_{d-1}},
\end{aligned}
$$

Proof Recall that $\left\{\phi_{i}\right\}_{i=1}^{d}$ is the first split sequence for $\Phi^{\Downarrow}$. Apply Lemma 8.1 to $\Phi^{\Downarrow}$ and use the data for $\Phi^{\Downarrow}$ in Proposition 3.6.

Lemma 9.5 (See $\left[18\right.$, Lemma 6.4]). For $1 \leq i \leq d$ the scalar $\phi_{i}$ is equal to each of the following four expressions:

$$
\begin{array}{ll}
\left(\theta_{i}^{*}-\theta_{i-1}^{*}\right) \sum_{h=0}^{i-1}\left(\theta_{d-h}-a_{h}\right), & \left(\theta_{i-1}^{*}-\theta_{i}^{*}\right) \sum_{h=i}^{d}\left(\theta_{d-h}-a_{h}\right), \\
\left(\theta_{d-i}-\theta_{d-i+1}\right) \sum_{h=0}^{i-1}\left(\theta_{h}^{*}-a_{d-h}^{*}\right), & \left(\theta_{d-i+1}-\theta_{d-i}\right) \sum_{h=i}^{d}\left(\theta_{h}^{*}-a_{d-h}^{*}\right) .
\end{array}
$$

Proof Apply Lemma 8.2 to $\Phi^{\Downarrow}$ and use the data for $\Phi^{\Downarrow}$ in Proposition 3.6.

Definition 9.6 (See [19, Definition 10.1]). By the parameter array of $\Phi$ we mean the sequence

$$
\left(\left\{\theta_{i}\right\}_{i=0}^{d} ;\left\{\theta_{i}^{*}\right\}_{i=0}^{d} ;\left\{\varphi_{i}\right\}_{i=1}^{d} ;\left\{\phi_{i}\right\}_{i=1}^{d}\right)
$$

where we recall that $\left\{\theta_{i}\right\}_{i=0}^{d}$ is the eigenvalue sequence of $\Phi,\left\{\theta_{i}^{*}\right\}_{i=0}^{d}$ is the dual eigenvalue sequence of $\Phi,\left\{\varphi_{i}\right\}_{i=1}^{d}$ is the first split sequence of $\Phi$, and $\left\{\phi_{i}\right\}_{i=1}^{d}$ is the second split sequence of $\Phi$.

Lemma 9.7 (See [18, Theorem 1.11]). The parameter arrays of

$$
\Phi, \quad \Phi^{\Downarrow}, \quad \Phi^{\downarrow}, \quad \Phi^{*}
$$

are related as follows. 


\begin{tabular}{ll}
\hline LS & Parameter array \\
\hline$\Phi$ & $\left(\left\{\theta_{i}\right\}_{i=0}^{d} ;\left\{\theta_{i}^{*}\right\}_{i=0}^{d} ;\left\{\varphi_{i}\right\}_{i=1}^{d} ;\left\{\phi_{i}\right\}_{i=1}^{d}\right)$ \\
$\Phi^{\Downarrow}$ & $\left(\left\{\theta_{d-i}\right\}_{i=0}^{d} ;\left\{\theta_{i}^{*}\right\}_{i=0}^{d} ;\left\{\phi_{i}\right\}_{i=1}^{d} ;\left\{\varphi_{i}\right\}_{i=1}^{d}\right)$ \\
$\Phi^{\downarrow}$ & $\left(\left\{\theta_{i}\right\}_{i=0}^{d} ;\left\{\theta_{d-i}^{*}\right\}_{i=0}^{d} ;\left\{\phi_{d-i+1}\right\}_{i=1}^{d} ;\left\{\varphi_{d-i+1}\right\}_{i=1}^{d}\right)$ \\
$\Phi^{*}$ & $\left(\left\{\theta_{i}^{*}\right\}_{i=0}^{d} ;\left\{\theta_{i}\right\}_{i=0}^{d} ;\left\{\varphi_{i}\right\}_{i=1}^{d} ;\left\{\phi_{d-i+1}\right\}_{i=1}^{d}\right)$ \\
\hline
\end{tabular}

Proof Use Proposition 3.6 and Lemmas 8.2, 9.5.

We mention a variation on Lemma 9.3.

Proposition 9.8 Two Leonard systems over $\mathbb{E}$ are isomorphic if and only if they have the same parameter array.

Proof By Lemma 9.3 and Definition 9.6.

\section{Statement of the Leonard System Classification}

In the following theorem we classify up to isomorphism the Leonard systems over $\mathbb{F}$.

Theorem 10.1 (See [18, Theorem 1.9]). Consider a sequence

$$
\left(\left\{\theta_{i}\right\}_{i=0}^{d} ;\left\{\theta_{i}^{*}\right\}_{i=0}^{d} ;\left\{\varphi_{i}\right\}_{i=1}^{d} ;\left\{\phi_{i}\right\}_{i=1}^{d}\right)
$$

of scalars in $\mathbb{F}$. Then there exists a Leonard system $\Phi$ over $\mathbb{F}$ with parameter array (21) if and only if the following conditions (PA1)-(PA5) hold:

(PA1) $\theta_{i} \neq \theta_{j}, \quad \theta_{i}^{*} \neq \theta_{j}^{*} \quad$ if $i \neq j, \quad(0 \leq i, j \leq d)$;

(PA2) $\varphi_{i} \neq 0, \quad \phi_{i} \neq 0 \quad(1 \leq i \leq d)$;

(PA3)

$$
\varphi_{i}=\phi_{1} \sum_{h=0}^{i-1} \frac{\theta_{h}-\theta_{d-h}}{\theta_{0}-\theta_{d}}+\left(\theta_{i}^{*}-\theta_{0}^{*}\right)\left(\theta_{i-1}-\theta_{d}\right) \quad(1 \leq i \leq d) ;
$$

(PA4)

$$
\phi_{i}=\varphi_{1} \sum_{h=0}^{i-1} \frac{\theta_{h}-\theta_{d-h}}{\theta_{0}-\theta_{d}}+\left(\theta_{i}^{*}-\theta_{0}^{*}\right)\left(\theta_{d-i+1}-\theta_{0}\right) \quad(1 \leq i \leq d)
$$

(PA5) the scalars

$$
\frac{\theta_{i-2}-\theta_{i+1}}{\theta_{i-1}-\theta_{i}}, \quad \frac{\theta_{i-2}^{*}-\theta_{i+1}^{*}}{\theta_{i-1}^{*}-\theta_{i}^{*}}
$$

are equal and independent of $i$ for $2 \leq i \leq d-1$.

Moreover, if $\Phi$ exists then $\Phi$ is unique up to isomorphism of Leonard systems.

The proof of Theorem 10.1 will be completed in Sect. 17. 
Definition 10.2 By a parameter array of diameter $d$ over $\mathbb{F}$, we mean a sequence (21) of scalars in $\mathbb{F}$ that satisfy (PA1)-(PA5).

Theorem 10.1 gives a bijection between the following two sets:

(i) the parameter arrays over $\mathbb{F}$ that have diameter $d$;

(ii) the isomorphism classes of Leonard systems over $\mathbb{F}$ that have diameter $d$.

We have a comment.

Lemma 10.3 For $d \geq 1$, a parameter array $\left(\left\{\theta_{i}\right\}_{i=0}^{d} ;\left\{\theta_{i}^{*}\right\}_{i=0}^{d} ;\left\{\varphi_{i}\right\}_{i=1}^{d} ;\left\{\phi_{i}\right\}_{i=1}^{d}\right)$ is uniquely determined by $\varphi_{1},\left\{\theta_{i}\right\}_{i=0}^{d},\left\{\theta_{i}^{*}\right\}_{i=0}^{d}$.

Proof By the nature of the equations (PA3), (PA4).

\section{Recurrent Sequences}

Throughout this section let $\left\{\theta_{i}\right\}_{i=0}^{d}$ denote scalars in $\mathbb{F}$.

Definition 11.1 (See [18, Definition 8.2]). Let $\beta, \gamma, \varrho$ denote scalars in $\mathbb{F}$.

(i) The sequence $\left\{\theta_{i}\right\}_{i=0}^{d}$ is said to be recurrent whenever $\theta_{i-1} \neq \theta_{i}$ for $2 \leq i \leq d-1$, and

$$
\frac{\theta_{i-2}-\theta_{i+1}}{\theta_{i-1}-\theta_{i}}
$$

is independent of $i$ for $2 \leq i \leq d-1$.

(ii) The sequence $\left\{\theta_{i}\right\}_{i=0}^{d}$ is said to be $\beta$-recurrent whenever

$$
\theta_{i-2}-(\beta+1) \theta_{i-1}+(\beta+1) \theta_{i}-\theta_{i+1}
$$

is zero for $2 \leq i \leq d-1$.

(iii) The sequence $\left\{\theta_{i}\right\}_{i=0}^{d}$ is said to be $(\beta, \gamma)$-recurrent whenever

$$
\theta_{i-1}-\beta \theta_{i}+\theta_{i+1}=\gamma
$$

for $1 \leq i \leq d-1$.

(iv) The sequence $\left\{\theta_{i}\right\}_{i=0}^{d}$ is said to be $(\beta, \gamma, \varrho)$-recurrent whenever

$$
\theta_{i-1}^{2}-\beta \theta_{i-1} \theta_{i}+\theta_{i}^{2}-\gamma\left(\theta_{i-1}+\theta_{i}\right)=\varrho
$$

for $1 \leq i \leq d$.

Lemma 11.2 The following are equivalent:

(i) the sequence $\left\{\theta_{i}\right\}_{i=0}^{d}$ is recurrent;

(ii) the scalars $\theta_{i-1} \neq \theta_{i}$ for $2 \leq i \leq d-1$, and there exists $\beta \in \mathbb{F}$ such that $\left\{\theta_{i}\right\}_{i=0}^{d}$ is $\beta$-recurrent. 
Suppose (i), (ii) hold, and that $d \geq 3$. Then the common value of (23) is equal to $\beta+1$.

Proof Routine.

Lemma 11.3 For $\beta \in \mathbb{F}$ the following are equivalent:

(i) the sequence $\left\{\theta_{i}\right\}_{i=0}^{d}$ is $\beta$-recurrent;

(ii) there exists $\gamma \in \mathbb{F}$ such that $\left\{\theta_{i}\right\}_{i=0}^{d}$ is $(\beta, \gamma)$-recurrent.

Proof (i) $\Rightarrow$ (ii) For $2 \leq i \leq d-1$, the expression (24) is zero by assumption, so

$$
\theta_{i-2}-\beta \theta_{i-1}+\theta_{i}=\theta_{i-1}-\beta \theta_{i}+\theta_{i+1} .
$$

The left-hand side of (25) is independent of $i$, and the result follows.

(ii) $\Rightarrow$ (i) For $2 \leq i \leq d-1$, subtract the equation (25) at $i$ from the corresponding equation obtained by replacing $i$ by $i-1$, to find (24) is zero.

Lemma 11.4 The following (i), (ii) hold for all $\beta, \gamma \in \mathbb{F}$.

(i) Suppose $\left\{\theta_{i}\right\}_{i=0}^{d}$ is $(\beta, \gamma)$-recurrent. Then there exists $\varrho \in \mathbb{F}$ such that $\left\{\theta_{i}\right\}_{i=0}^{d}$ is $(\beta, \gamma, \varrho)$-recurrent.

(ii) Suppose $\left\{\theta_{i}\right\}_{i=0}^{d}$ is $(\beta, \gamma, \varrho)$-recurrent, and that $\theta_{i-1} \neq \theta_{i+1}$ for $1 \leq i \leq d-1$. Then $\left\{\theta_{i}\right\}_{i=0}^{d}$ is $(\beta, \gamma)$-recurrent.

Proof Let $p_{i}$ denote the expression on the left in (26), and observe

$$
p_{i}-p_{i+1}=\left(\theta_{i-1}-\theta_{i+1}\right)\left(\theta_{i-1}-\beta \theta_{i}+\theta_{i+1}-\gamma\right)
$$

for $1 \leq i \leq d-1$. Assertions (i), (ii) are both routine consequences of this.

\section{Recurrent Sequences in Closed Form}

In this section, we obtain some formula involving recurrent sequences. Let $\overline{\mathbb{F}}$ denote the algebraic closure of $\mathbb{F}$. For $q \in \overline{\mathbb{F}}$ let $\mathbb{F}[q]$ denote the field extension of $\mathbb{F}$ generated by $q$.

Throughout this section let $\beta$ and $\left\{\theta_{i}\right\}_{i=0}^{d}$ denote scalars in $\mathbb{F}$.

Lemma 12.1 Assume that $\left\{\theta_{i}\right\}_{i=0}^{d}$ is $\beta$-recurrent. Then the following (i)-(iii) hold.

(i) Suppose $\beta \neq 2, \beta \neq-2$, and pick $0 \neq q \in \overline{\mathbb{F}}$ such that $q+q^{-1}=\beta$. Then there exist scalars $\alpha_{1}, \alpha_{2}, \alpha_{3}$ in $\mathbb{E}[q]$ such that

$$
\theta_{i}=\alpha_{1}+\alpha_{2} q^{i}+\alpha_{3} q^{-i} \quad(0 \leq i \leq d) .
$$

(ii) Suppose $\beta=2$. Then there exist $\alpha_{1}, \alpha_{2}, \alpha_{3}$ in $\mathbb{F}$ such that 


$$
\theta_{i}=\alpha_{1}+\alpha_{2} i+\alpha_{3} i(i-1) / 2 \quad(0 \leq i \leq d) .
$$

(iii) Suppose $\beta=-2$ and $\operatorname{char}(\mathbb{F}) \neq 2$. Then there exist $\alpha_{1}, \alpha_{2}, \alpha_{3}$ in $\mathbb{F}$ such that

$$
\theta_{i}=\alpha_{1}+\alpha_{2}(-1)^{i}+\alpha_{3} i(-1)^{i} \quad(0 \leq i \leq d) .
$$

Referring to case (ii) above, if $\operatorname{char}(\mathbb{F})=2$ then we interpret the expression $i(i-$ $1) / 2$ as 0 if $i=0$ or $i=1(\bmod 4)$, and as 1 if $i=2$ or $i=3(\bmod 4)$.

\section{Proof}

(i) We assume $d \geq 2$; otherwise the result is trivial. Let $q$ be given, and consider the equations (27) for $i=0,1,2$. These equations are linear in $\alpha_{1}, \alpha_{2}, \alpha_{3}$. We routinely find the coefficient matrix is nonsingular, so there exist $\alpha_{1}, \alpha_{2}, \alpha_{3}$ in $\mathbb{F}[q]$ such that (27) holds for $i=0,1,2$. Using these scalars, let $\varepsilon_{i}$ denote the left-hand side of (27) minus the righthand side of (27), for $0 \leq i \leq d$. On one hand $\varepsilon_{0}, \varepsilon_{1}, \varepsilon_{2}$ are zero from the construction. On the other hand, one readily checks

$$
\varepsilon_{i-2}-(\beta+1) \varepsilon_{i-1}+(\beta+1) \varepsilon_{i}-\varepsilon_{i+1}=0
$$

for $2 \leq i \leq d-1$. By these comments $\varepsilon_{i}=0$ for $0 \leq i \leq d$, and the result follows.

(ii), (iii) Similar to the proof of (i) above.

Lemma 12.2 Assume that $\left\{\theta_{i}\right\}_{i=0}^{d}$ are mutually distinct and $\beta$-recurrent. Then $(i)-$ (iv) hold below.

(i) Suppose $\beta \neq 2, \beta \neq-2$, and pick $0 \neq q \in \overline{\mathbb{F}}$ such that $q+q^{-1}=\beta$. Then $q^{i} \neq 1$ for $1 \leq i \leq d$.

(ii) Suppose $\beta=2$ and $\operatorname{char}(\mathbb{F})=p, p \geq 3$. Then $d<p$.

(iii) Suppose $\beta=-2$ and $\operatorname{char}(\mathbb{F})=p, p \geq 3$. Then $d<2 p$.

(iv) Suppose $\beta=0$ and $\operatorname{char}(\mathbb{F})=2$. Then $d \leq 3$.

\section{Proof}

(i) Using (27), we find $q^{i}=1$ implies $\theta_{i}=\theta_{0}$ for $1 \leq i \leq d$.

(ii) Suppose $d \geq p$. Setting $i=p$ in (28) and recalling that $p$ is congruent to 0 modulo $p$, we obtain $\theta_{p}=\theta_{0}$, a contradiction. Hence $d<p$.

(iii) Suppose $d \geq 2 p$. Setting $i=2 p$ in (29) and recalling that $p$ is congruent to 0 modulo $p$, we obtain $\theta_{2 p}=\theta_{0}$, a contradiction. Hence $d<2 p$.

(iv) Suppose $d \geq 4$. Setting $i=4$ in (28), we find $\theta_{4}=\theta_{0}$ in view of the comment at the end of Lemma 12.1. This is a contradiction, so $d \leq 3$. 
Lemma 12.3 (See [18, Lemma 9.4]). Assume that $\left\{\theta_{i}\right\}_{i=0}^{d}$ are mutually distinct and $\beta$-recurrent. Pick any integers $i, j, r, s(0 \leq i, j, r, s \leq d)$ such that $i+j=r+s$, $r \neq s$. Then (i)-(iv) hold below.

(i) Suppose $\beta \neq 2, \beta \neq-2$. Then

$$
\frac{\theta_{i}-\theta_{j}}{\theta_{r}-\theta_{s}}=\frac{q^{i}-q^{j}}{q^{r}-q^{s}},
$$

where $q+q^{-1}=\beta$.

(ii) Suppose $\beta=2$ and $\operatorname{char}(\mathbb{F}) \neq 2$. Then

$$
\frac{\theta_{i}-\theta_{j}}{\theta_{r}-\theta_{s}}=\frac{i-j}{r-s}
$$

(iii) Suppose $\beta=-2$ and $\operatorname{char}(\mathbb{F}) \neq 2$. Then

$$
\frac{\theta_{i}-\theta_{j}}{\theta_{r}-\theta_{s}}=\left\{\begin{array}{cc}
(-1)^{i+r} \frac{i-j}{r-s}, & \text { if } i+j \text { is even } \\
(-1)^{i+r}, & \text { if } i+j \text { is odd }
\end{array}\right.
$$

(iv) Suppose $\beta=0$ and $\operatorname{char}(\mathbb{F})=2$. Then

$$
\frac{\theta_{i}-\theta_{j}}{\theta_{r}-\theta_{s}}= \begin{cases}0, & \text { if } i=j \\ 1, & \text { if } i \neq j\end{cases}
$$

Proof To get (i), evaluate the left-hand side of (30) using (27), and simplify the result. The cases (ii)-(iv) are similar.

\section{A Sum}

Throughout this section assume $d \geq 1$. Let $\beta$ and $\left\{\theta_{i}\right\}_{i=0}^{d}$ denote scalars in $\mathbb{F}$ with $\left\{\theta_{i}\right\}_{i=0}^{d}$ mutually distinct.

We consider the sums

$$
\sum_{h=0}^{i-1} \frac{\theta_{h}-\theta_{d-h}}{\theta_{0}-\theta_{d}}
$$

where $0 \leq i \leq d+1$. Denoting the sum in (34) by $\vartheta_{i}$, we have

$$
\vartheta_{0}=0, \quad \vartheta_{1}=1, \quad \vartheta_{d}=1, \quad \vartheta_{d+1}=0 .
$$

Moreover

$$
\vartheta_{i}=\vartheta_{d-i+1} \quad(0 \leq i \leq d+1)
$$

The sums (34) play an important role a bit later, so we will examine them carefully. 
We begin by giving explicit formulas for the sums (34) under the assumption that $\left\{\theta_{i}\right\}_{i=0}^{d}$ is $\beta$-recurrent. To avoid trivialities we assume that $d \geq 3$.

Lemma 13.1 (See [18, Lemma 10.2]). Assume that $\left\{\theta_{i}\right\}_{i=0}^{d}$ are mutually distinct and $\beta$-recurrent. Further assume that $d \geq 3$. Then for $0 \leq i \leq d+1$ we have the following.

(i) Suppose $\beta \neq 2, \beta \neq-2$. Then

$$
\sum_{h=0}^{i-1} \frac{\theta_{h}-\theta_{d-h}}{\theta_{0}-\theta_{d}}=\frac{q^{i}-1}{q-1} \frac{q^{d-i+1}-1}{q^{d}-1}
$$

where $q+q^{-1}=\beta$.

(ii) Suppose $\beta=2$ and $\operatorname{char}(\mathbb{F}) \neq 2$. Then

$$
\sum_{h=0}^{i-1} \frac{\theta_{h}-\theta_{d-h}}{\theta_{0}-\theta_{d}}=\frac{i(d-i+1)}{d}
$$

(iii) Suppose $\beta=-2, \operatorname{char}(\mathbb{F}) \neq 2$, and $d$ odd. Then

$$
\sum_{h=0}^{i-1} \frac{\theta_{h}-\theta_{d-h}}{\theta_{0}-\theta_{d}}= \begin{cases}0, & \text { if } i \text { is even } \\ 1, & \text { if } i \text { is odd }\end{cases}
$$

(iv) Suppose $\beta=-2$, $\operatorname{char}(\mathbb{F}) \neq 2$, and $d$ even. Then

$$
\sum_{h=0}^{i-1} \frac{\theta_{h}-\theta_{d-h}}{\theta_{0}-\theta_{d}}=\left\{\begin{array}{cc}
i / d, & \text { if } i \text { is even } \\
(d-i+1) / d, & \text { if } i \text { is odd }
\end{array}\right.
$$

(v) Suppose $\beta=0$, $\operatorname{char}(\mathbb{F})=2$, and $d=3$. Then

$$
\sum_{h=0}^{i-1} \frac{\theta_{h}-\theta_{d-h}}{\theta_{0}-\theta_{d}}=\left\{\begin{array}{cc}
0, & \text { if } i \text { is even } \\
1, & \text { if } i \text { is odd }
\end{array}\right.
$$

Proof The above sums can be computed directly from Lemma 12.3.

Note 13.2 Referring to Lemma 13.1, the cases (iii), (iv) can be handled in the following uniform way. Suppose $\beta=-2$ and $\operatorname{char}(\mathbb{F}) \neq 2$. Then for $0 \leq i \leq d+1$,

$$
\sum_{h=0}^{i-1} \frac{\theta_{h}-\theta_{d-h}}{\theta_{0}-\theta_{d}}=\frac{2 d+1+(2 i-2 d-1)(-1)^{i}+(-1)^{d}+(2 i-1)(-1)^{i+d}}{4 d} .
$$

We make an observation.

Lemma 13.3 Assume that $\left\{\theta_{i}\right\}_{i=0}^{d}$ are mutually distinct and $\beta$-recurrent. Define 


$$
\vartheta_{i}=\sum_{h=0}^{i-1} \frac{\theta_{h}-\theta_{d-h}}{\theta_{0}-\theta_{d}} \quad(0 \leq i \leq d+1) .
$$

Then the sequence $\left\{\vartheta_{i}\right\}_{i=0}^{d+1}$ is $\beta$-recurrent.

Proof For $d=1$ there is nothing to prove. For $d=2$ we have

$$
\vartheta_{0}-(\beta+1) \vartheta_{1}+(\beta+1) \vartheta_{2}-\vartheta_{3}=0
$$

since

$$
\vartheta_{0}=0, \quad \vartheta_{1}=1, \quad \vartheta_{2}=1, \quad \vartheta_{3}=0 .
$$

For $d \geq 3$ the result is obtained by examining the cases in Lemma 13.1.

Proposition 13.4 Assume that $\left\{\theta_{i}\right\}_{i=0}^{d}$ are mutually distinct and $\beta$-recurrent. Then for scalars $\left\{\vartheta_{i}\right\}_{i=0}^{d+1}$ in $\mathbb{F}$ the following are equivalent:

(i) $\vartheta_{i}=\vartheta_{1} \sum_{h=0}^{i-1} \frac{\theta_{h}-\theta_{d-h}}{\theta_{0}-\theta_{d}} \quad(0 \leq i \leq d+1)$;

(ii) the sequence $\left\{\vartheta_{i}\right\}_{i=0}^{d+1}$ is $\beta$-recurrent and

$$
\vartheta_{0}=0, \quad \vartheta_{1}=\vartheta_{d}, \quad \vartheta_{d+1}=0 .
$$

Proof (i) $\Rightarrow\left(\right.$ ii) The sequence $\left\{\vartheta_{i}\right\}_{i=0}^{d+1}$ is $\beta$-recurrent by Lemma 13.3. Condition (43) follows from (35).

(ii) $\Rightarrow$ (i) Define

$$
\Delta_{i}=\vartheta_{i}-\vartheta_{1} \sum_{h=0}^{i-1} \frac{\theta_{h}-\theta_{d-h}}{\theta_{0}-\theta_{d}} \quad(0 \leq i \leq d+1) .
$$

We show that $\Delta_{i}=0$ for $0 \leq i \leq d+1$. By construction

$$
\Delta_{0}=0, \quad \Delta_{1}=0, \quad \Delta_{d}=0, \quad \Delta_{d+1}=0 .
$$

For the rest of the proof we assume that $d \geq 3$; otherwise we are done. By construction and Lemma 13.3, the sequence $\left\{\Delta_{i}\right\}_{i=0}^{d+1}$ is $\beta$-recurrent. We break the argument into cases.

Case $\beta \neq 2, \beta \neq-2$. Pick $0 \neq q \in \overline{\mathbb{F}}$ such that $q+q^{-1}=\beta$. There exist $\alpha_{1}, \alpha_{2}$, $\alpha_{3}$ in $\mathbb{F}[q]$ such that

$$
\Delta_{i}=\alpha_{1}+\alpha_{2} q^{i}+\alpha_{3} q^{-i} \quad(0 \leq i \leq d+1) .
$$

Since $\left\{\theta_{i}\right\}_{i=0}^{d}$ are mutually distinct and $\beta$-recurrent, we have $q^{i} \neq 1$ for $1 \leq i \leq d$. The first three equations in (44) give 


$$
\left(\begin{array}{l}
0 \\
0 \\
0
\end{array}\right)=\left(\begin{array}{ccc}
1 & 1 & 1 \\
1 & q & q^{-1} \\
1 & q^{d} & q^{-d}
\end{array}\right)\left(\begin{array}{l}
\alpha_{1} \\
\alpha_{2} \\
\alpha_{3}
\end{array}\right) .
$$

For the above equation the coefficient matrix has determinant

$$
(q-1)\left(q^{d}-1\right)\left(q^{d-1}-1\right) q^{-d},
$$

which is nonzero. Therefore the coefficient matrix is invertible, so each of $\alpha_{1}, \alpha_{2}, \alpha_{3}$ is zero. Consequently $\Delta_{i}=0$ for $0 \leq i \leq d+1$.

Case $\beta=2$ and $\operatorname{char}(\mathbb{F}) \neq 2$. There exist $\alpha_{1}, \alpha_{2}, \alpha_{3}$ in $\mathbb{F}$ such that

$$
\Delta_{i}=\alpha_{1}+\alpha_{2} i+\alpha_{3} i(i-1) / 2 \quad(0 \leq i \leq d+1) .
$$

Since $\left\{\theta_{i}\right\}_{i=0}^{d}$ are mutully distinct and $\beta$-recurrent, we have $\operatorname{char}(\mathbb{F})=0$ or $\operatorname{char}(\mathbb{F})=p$ with $d<p$. The first three equations in (44) give

$$
\left(\begin{array}{l}
0 \\
0 \\
0
\end{array}\right)=\left(\begin{array}{ccc}
1 & 0 & 0 \\
1 & 1 & 0 \\
1 & d & d(d-1) / 2
\end{array}\right)\left(\begin{array}{l}
\alpha_{1} \\
\alpha_{2} \\
\alpha_{3}
\end{array}\right) .
$$

For the above equation the coefficient matrix has determinant $d(d-1) / 2$, which is nonzero. Therefore the coefficient matrix is invertible, so each of $\alpha_{1}, \alpha_{2}, \alpha_{3}$ is zero. Consequently $\Delta_{i}=0$ for $0 \leq i \leq d+1$.

Case $\beta=-2$ and $\operatorname{char}(\mathbb{F}) \neq 2$. There exist $\alpha_{1}, \alpha_{2}, \alpha_{3}$ in $\mathbb{F}$ such that

$$
\Delta_{i}=\alpha_{1}+\alpha_{2}(-1)^{i}+\alpha_{3} i(-1)^{i} \quad(0 \leq i \leq d+1) .
$$

Since $\left\{\theta_{i}\right\}_{i=0}^{d}$ are mutully distinct and $\beta$-recurrent, we have

$$
\operatorname{char}(\mathbb{F})=0 \quad \text { or } \quad \operatorname{char}(\mathbb{F})=p, \quad d<2 p .
$$

The first three equations in (44) give

$$
\left(\begin{array}{l}
0 \\
0 \\
0
\end{array}\right)=\left(\begin{array}{ccc}
1 & 1 & 0 \\
1 & -1 & -1 \\
1 & (-1)^{d} & d(-1)^{d}
\end{array}\right)\left(\begin{array}{l}
\alpha_{1} \\
\alpha_{2} \\
\alpha_{3}
\end{array}\right) .
$$

For the above equation, consider the determinant of the coefficient matrix. For even $d=2 n$ this determinant is $-2^{2} n$, and for odd $d=2 n+1$ this determinant is $2^{2} n$. Note that $2 \neq 0$ in $\mathbb{F}$ since $\operatorname{char}(\mathbb{F}) \neq 2$. For either parity of $d$ we have $n \neq 0$ in $\mathbb{F}$ by (45). So for either parity of $d$ the determinant is nonzero. Therefore the coefficient matrix is invertible, so each of $\alpha_{1}, \alpha_{2}, \alpha_{3}$ is zero. Consequently $\Delta_{i}=0$ for $0 \leq i \leq d+1$.

Case $\beta=0$ and $\operatorname{char}(\mathbb{F})=2$. We have $d=3$ by Lemma 12.2(iv). There exist $\alpha_{1}$, $\alpha_{2}, \alpha_{3}$ in $\mathbb{F}$ such that 


$$
\Delta_{i}=\alpha_{1}+\alpha_{2} i+\alpha_{3} i(i-1) / 2 \quad(0 \leq i \leq 4),
$$

where $i(i-1) / 2$ is interpreted at the end of Lemma 12.1. The first three equations in (44) give

$$
\left(\begin{array}{l}
0 \\
0 \\
0
\end{array}\right)=\left(\begin{array}{lll}
1 & 0 & 0 \\
1 & 1 & 0 \\
1 & 1 & 1
\end{array}\right)\left(\begin{array}{l}
\alpha_{1} \\
\alpha_{2} \\
\alpha_{3}
\end{array}\right) .
$$

In the above equation the coefficient matrix is invertible, so each of $\alpha_{1}, \alpha_{2}, \alpha_{3}$ is zero. Consequently $\Delta_{i}=0$ for $0 \leq i \leq d+1$.

\section{The Polynomial $P(x, y)$}

Let $\beta, \gamma, \varrho$ denote scalars in $\mathbb{F}$, and consider a polynomial in two variables

$$
P(x, y)=x^{2}-\beta x y+y^{2}-\gamma(x+y)-\varrho .
$$

Note that $P(x, y)=P(y, x)$. Let $\left\{\theta_{i}\right\}_{i=0}^{d}$ denote scalars in $\mathbb{F}$.

Lemma 14.1 The following are equivalent:

(i) $P\left(\theta_{i-1}, \theta_{i}\right)=0$ for $1 \leq i \leq d$;

(ii) the sequence $\left\{\theta_{i}\right\}_{i=0}^{d}$ is $(\beta, \gamma, \varrho)$-recurrent.

Proof By Definition 11.1(iv).

Proposition 14.2 Assume that $\left\{\theta_{i}\right\}_{i=0}^{d}$ are mutually distinct and $(\beta, \gamma, \varrho)$-recurrent. Then the following hold:

(i) $P\left(x, \theta_{j}\right)=\left(x-\theta_{j-1}\right)\left(x-\theta_{j+1}\right) \quad(1 \leq j \leq d-1)$;

(ii) for $0 \leq i, j \leq d, P\left(\theta_{i}, \theta_{j}\right)=0$ implies $|i-j|=1$ or $i, j \in\{0, d\}$.

\section{Proof}

(i) The polynomial $P\left(x, \theta_{j}\right)$ is monic in $x$, and has roots $\theta_{j-1}, \theta_{j+1}$ by Lemma 14.1.

(ii) Assume that $P\left(\theta_{i}, \theta_{j}\right)=0$. Also assume that $1 \leq i \leq d-1$ or $1 \leq j \leq d-1$; otherwise $i, j \in\{0, d\}$ and we are done. Interchanging $i, j$ if necessary, we may assume that $1 \leq j \leq d-1$. Using (i) we have $0=P\left(\theta_{i}, \theta_{j}\right)=$ $\left(\theta_{i}-\theta_{j-1}\right)\left(\theta_{i}-\theta_{j+1}\right)$. Therefore $i=j-1$ or $i=j+1$, so $|i-j|=1$.

\section{The Tridiagonal Relations}

In this section, we consider a Leonard system 


$$
\Phi=\left(A ;\left\{E_{i}\right\}_{i=0}^{d} ; A^{*} ;\left\{E_{i}^{*}\right\}_{i=0}^{d}\right)
$$

on $V$, with eigenvalue sequence $\left\{\theta_{i}\right\}_{i=0}^{d}$ and dual eigenvalue sequence $\left\{\theta_{i}^{*}\right\}_{i=0}^{d}$. Our goal is to prove the following result.

Theorem 15.1 (See [18, Theorem 1.12]). There exists a sequence of scalars $\beta, \gamma, \gamma^{*}, \varrho, \varrho^{*}$ taken from $\mathbb{F}$ such that both

$$
\begin{aligned}
& 0=\left[A, A^{2} A^{*}-\beta A A^{*} A+A^{*} A^{2}-\gamma\left(A A^{*}+A^{*} A\right)-\varrho A^{*}\right], \\
& 0=\left[A^{*}, A^{* 2} A-\beta A^{*} A A^{*}+A A^{* 2}-\gamma^{*}\left(A^{*} A+A A^{*}\right)-\varrho^{*} A\right] .
\end{aligned}
$$

The sequence is unique if $d \geq 3$.

The relations (TD1), (TD2) are called the tridiagonal relations. They are displayed in [16, Lemma 5.4] and examined carefully in [17].

Lemma 15.2 For $\beta, \gamma, \varrho \in \mathbb{F}$ the following are equivalent:

(i) the scalars $\beta, \gamma, \varrho$ satisfy (TD1);

(ii) the sequence $\left\{\theta_{i}\right\}_{i=0}^{d}$ is $(\beta, \gamma, \varrho)$-recurrent.

Proof Let $C$ denote the expression on the right in (TD1). We have

$$
C=\sum_{i=0}^{d} \sum_{j=0}^{d} E_{i} C E_{j} .
$$

For $0 \leq i, j \leq d$,

$$
E_{i} C E_{j}=\left(\theta_{i}-\theta_{j}\right) P\left(\theta_{i}, \theta_{j}\right) E_{i} A^{*} E_{j}
$$

where $P$ is from (46).

(i) $\Rightarrow$ (ii) We have $C=0$. So for $1 \leq j \leq d$,

$$
0=E_{j-1} C E_{j}=\left(\theta_{j-1}-\theta_{j}\right) P\left(\theta_{j-1}, \theta_{j}\right) E_{j-1} A^{*} E_{j} .
$$

By construction $\theta_{j-1} \neq \theta_{j}$ and $E_{j-1} A^{*} E_{j} \neq 0$. Therefore $P\left(\theta_{j-1}, \theta_{j}\right)=0$. Consequently the sequence $\left\{\theta_{i}\right\}_{i=0}^{d}$ is $(\beta, \gamma, \varrho)$-recurrent.

(ii) $\Rightarrow$ (i) For $0 \leq i, j \leq d$ the right-hand side of (47) has at least one zero factor, so $E_{i} C E_{j}=0$. Consequently $C=0$.

Lemma 15.3 The following (i)-(iii) hold for $0 \leq i, j \leq d$ :

(i) $E_{i}^{*} A^{r} E_{j}^{*}=0$ for $0 \leq r<|i-j|$;

(ii) $E_{i}^{*} A^{r} E_{j}^{*} \neq 0$ for $r=|i-j|$;

(iii) for $0 \leq r, s \leq d$, 


$$
E_{i}^{*} A^{r} A^{*} A^{s} E_{j}^{*}=\left\{\begin{array}{cl}
\theta_{j+s}^{*} E_{i}^{*} A^{r+s} E_{j}^{*}, & \text { if } i-j=r+s \\
\theta_{j-s}^{*} E_{i}^{*} A^{r+s} E_{j}^{*}, & \text { if } j-i=r+s \\
0, & \text { if }|i-j|>r+s .
\end{array}\right.
$$

Proof For $0 \leq i \leq d$ pick $0 \neq v_{i} \in E_{i}^{*} V$. So $\left\{v_{i}\right\}_{i=0}^{d}$ is a basis for $V$. Without loss of generality, we may identify each $X \in \operatorname{End}(V)$ with the matrix in $\operatorname{Mat}_{d+1}(\mathbb{F})$ that represents $X$ with respect to $\left\{v_{i}\right\}_{i=0}^{d}$. From this point of view, $A$ is irreducible tridiagonal and $A^{*}=\operatorname{diag}\left(\theta_{0}^{*}, \theta_{1}^{*}, \ldots, \theta_{d}^{*}\right)$. Moreover for $0 \leq i \leq d$, the matrix $E_{i}^{*}$ is diagonal with $(i, i)$-entry 1 and all other entries 0 . Using these matrix representations, one routinely verifies the assertions (i)-(iii) in the lemma statement.

Recall that $\mathcal{D}$ is the subalgebra of $\operatorname{End}(V)$ generated by $A$.

\section{Lemma 15.4 Define}

$$
L_{i}=E_{0}+E_{1}+\cdots+E_{i} \quad(0 \leq i \leq d) .
$$

Then

(i) $\quad\left\{L_{i}\right\}_{i=0}^{d}$ is a basis for the vector space $\mathcal{D}$;

(ii) $L_{d}=I$;

(iii) for $0 \leq i \leq d-1$,

$$
L_{i} A^{*}-A^{*} L_{i}=E_{i} A^{*} E_{i+1}-E_{i+1} A^{*} E_{i} .
$$

\section{Proof}

(i) Since $\left\{E_{i}\right\}_{i=0}^{d}$ is a basis for $\mathcal{D}$.

(ii) Since $I=\sum_{i=0}^{d} E_{i}$.

(iii) For $0 \leq j \leq d-1$ we have

$$
\begin{aligned}
E_{j} A^{*} & =E_{j} A^{*}\left(E_{0}+\cdots+E_{d}\right) \\
& =E_{j} A^{*} E_{j-1}+E_{j} A^{*} E_{j}+E_{j} A^{*} E_{j+1},
\end{aligned}
$$

where $E_{-1}=0$. Similarly for $0 \leq j \leq d-1$,

$$
A^{*} E_{j}=E_{j-1} A^{*} E_{j}+E_{j} A^{*} E_{j}+E_{j+1} A^{*} E_{j} .
$$

Sum both (48) and (49) over $j=0,1, \ldots, i$ and take the difference between these two sums.

Lemma 15.5 We have

$$
\operatorname{Span}\left\{X A^{*} Y-Y A^{*} X \mid X, Y \in \mathcal{D}\right\}=\left\{Z A^{*}-A^{*} Z \mid Z \in \mathcal{D}\right\} .
$$


Proof Using Lemma 15.4 we obtain

$$
\begin{aligned}
\operatorname{Span}\left\{X A^{*} Y\right. & \left.-Y A^{*} X \mid X, Y \in \mathcal{D}\right\} \\
& =\operatorname{Span}\left\{E_{i} A^{*} E_{j}-E_{j} A^{*} E_{i} \mid 0 \leq i, j \leq d\right\} \\
& =\operatorname{Span}\left\{E_{i} A^{*} E_{i+1}-E_{i+1} A^{*} E_{i} \mid 0 \leq i \leq d-1\right\} \\
& =\operatorname{Span}\left\{L_{i} A^{*}-A^{*} L_{i} \mid 0 \leq i \leq d-1\right\} \\
& =\left\{Z A^{*}-A^{*} Z \mid Z \in \mathcal{D}\right\}
\end{aligned}
$$

Proof of Theorem 15.1 First assume that $d \geq 3$. By Lemma 15.5 (with $X=A^{2}$ and $Y=A$ ) there exists $Z \in \mathcal{D}$ such that

$$
A^{2} A^{*} A-A A^{*} A^{2}=Z A^{*}-A^{*} Z \text {. }
$$

Since $\left\{A^{i}\right\}_{i=0}^{d}$ is a basis for $\mathcal{D}$, there exists a polynomial $f \in \mathbb{F}[\lambda]$ such that $\operatorname{deg}(f) \leq d$ and $Z=f(A)$. Let $k$ denote the degree of $f$.

We show that $k=3$. We first assume that $k<3$ and get a contradiction. We multiply each term in (50) on the left by $E_{3}^{*}$ and on the right by $E_{0}^{*}$. We evaluate the result using Lemma 15.3 to find $\left(\theta_{1}^{*}-\theta_{2}^{*}\right) E_{3}^{*} A^{3} E_{0}^{*}=0$. The scalar $\theta_{1}^{*}-\theta_{2}^{*}$ is nonzero, and $E_{3}^{*} A^{3} E_{0}^{*} \neq 0$ by Lemma 15 .3(ii). Therefore $\left(\theta_{1}^{*}-\theta_{2}^{*}\right) E_{3}^{*} A^{3} E_{0}^{*} \neq 0$ for a contradiction. We have shown $k \geq 3$. Let $c$ denote the coefficient of $\lambda^{k}$ in $f$. By construction $c \neq 0$.

Next we assume that $k>3$ and get a contradiction. We multiply each term in (50) on the left by $E_{k}^{*}$ and on the right by $E_{0}^{*}$. We evaluate the result using Lemma 15.3 to find $0=c\left(\theta_{0}^{*}-\theta_{k}^{*}\right) E_{k}^{*} A^{k} E_{0}^{*}$. The scalars $c$ and $\theta_{0}^{*}-\theta_{k}^{*}$ are nonzero, and $E_{k}^{*} A^{k} E_{0}^{*} \neq 0$ by Lemma $15.3(\mathrm{ii})$. Therefore $0 \neq c\left(\theta_{0}^{*}-\theta_{k}^{*}\right) E_{k}^{*} A^{k} E_{0}^{*}$ for a contradiction. We have shown $k=3$.

Define $\beta=c^{-1}-1$, so $\beta+1=c^{-1}$. Multiply each term in (50) by $c^{-1}$. The result is

$$
(\beta+1)\left(A^{2} A^{*} A-A A^{*} A^{2}\right)=A^{3} A^{*}-A^{*} A^{3}-\gamma\left(A^{2} A^{*}-A^{*} A^{2}\right)-\varrho\left(A A^{*}-A^{*} A\right),
$$

where $\gamma, \varrho \in \mathbb{F}$. The equation (51) is (TD1) in disguise; it is (TD1) with the commutator expanded. Therefore $\beta, \gamma, \varrho$ satisfy (TD1). Concerning (TD2), pick any integer $i(2 \leq i \leq d-1)$. We multiply each term in (51) on the left by $E_{i-2}^{*}$ and on the right by $E_{i+1}^{*}$. We evaluate the result using Lemma 15.3 to find that $E_{i-2}^{*} A^{3} E_{i+1}^{*}$ times

$$
\theta_{i-2}^{*}-(\beta+1) \theta_{i-1}^{*}+(\beta+1) \theta_{i}^{*}-\theta_{i+1}^{*}
$$

is zero. We have $E_{i-2}^{*} A^{3} E_{i+1}^{*} \neq 0$ by Lemma $15.3(\mathrm{ii})$, so (52) is zero. Thus the sequence $\left\{\theta_{i}^{*}\right\}_{i=0}^{d}$ is $\beta$-recurrent. By Lemma 11.3 there exists $\gamma^{*} \in \mathbb{F}$ such that $\left\{\theta_{i}^{*}\right\}_{i=0}^{d}$ is $\left(\beta, \gamma^{*}\right)$-recurrent. By Lemma 11.4(i) there exists $\varrho^{*} \in \mathbb{F}$ such that $\left\{\theta_{i}^{*}\right\}_{i=0}^{d}$ is $\left(\beta, \gamma^{*}, \varrho^{*}\right)$-recurrent. By this and Lemma 15.2 (applied to $\left.\Phi^{*}\right)$ we see that $\beta, \gamma^{*}, \varrho^{*}$ 
satisfy (TD2).

We have obtained scalars $\beta, \gamma, \gamma^{*}, \varrho, \varrho^{*}$ in $\mathbb{F}$ that satisfy (TD1), (TD2). Next we show that these scalars are unique. Let $\beta, \gamma, \gamma^{*}, \varrho, \varrho^{*}$ denote any scalars in $\mathbb{F}$ that satisfy (TD1), (TD2). By Lemma 15.2 the sequence $\left\{\theta_{i}\right\}_{i=0}^{d}$ is $(\beta, \gamma, \varrho)$-recurrent. By Lemma 11.4(ii) the sequence $\left\{\theta_{i}\right\}_{i=0}^{d}$ is $(\beta, \gamma)$-recurrent. By Lemma 11.3 the sequence $\left\{\theta_{i}\right\}_{i=0}^{d}$ is $\beta$-recurrent. Also by Lemma 15.2 the sequence $\left\{\theta_{i}^{*}\right\}_{i=0}^{d}$ is $\left(\beta, \gamma^{*}, \varrho^{*}\right)$-recurrent. By Lemma 11.4 (ii) the sequence $\left\{\theta_{i}^{*}\right\}_{i=0}^{d}$ is $\left(\beta, \gamma^{*}\right)$-recurrent. By Lemma 11.3 the sequence $\left\{\theta_{i}^{*}\right\}_{i=0}^{d}$ is $\beta$-recurrent. By these comments and Definition 11.1,

- the scalars

$$
\frac{\theta_{i-2}-\theta_{i+1}}{\theta_{i-1}-\theta_{i}}, \quad \frac{\theta_{i-2}^{*}-\theta_{i+1}^{*}}{\theta_{i-1}^{*}-\theta_{i}^{*}}
$$

are both equal to $\beta+1$ for $2 \leq i \leq d-1$;

- $\gamma=\theta_{i-1}-\beta \theta_{i}+\theta_{i+1} \quad(1 \leq i \leq d-1)$,

- $\gamma^{*}=\theta_{i-1}^{*}-\beta \theta_{i}^{*}+\theta_{i+1}^{*} \quad(1 \leq i \leq d-1)$,

- $\varrho=\theta_{i-1}^{2}-\beta \theta_{i-1} \theta_{i}+\theta_{i}^{2}-\gamma\left(\theta_{i-1}+\theta_{i}\right) \quad(1 \leq i \leq d)$,

- $\varrho^{*}=\theta_{i-1}^{* 2}-\beta \theta_{i-1}^{*} \theta_{i}^{*}+\theta_{i}^{* 2}-\gamma^{*}\left(\theta_{i-1}^{*}+\theta_{i}^{*}\right) \quad(1 \leq i \leq d)$.

The above equations show that $\beta, \gamma, \gamma^{*}, \varrho, \varrho^{*}$ are unique. We have proved the theorem under the assumption $d \geq 3$.

Next assume that $d \leq 2$. Pick any $\beta \in \mathbb{F}$. For $d=2$ define $\gamma=\theta_{0}-\beta \theta_{1}+\theta_{2}$ and for $d \leq 1$ pick any $\gamma \in \mathbb{F}$. For $d \geq 1$ define

$$
\varrho=\theta_{0}^{2}-\beta \theta_{0} \theta_{1}+\theta_{1}^{2}-\gamma\left(\theta_{0}+\theta_{1}\right)
$$

and for $d=0$ pick any $\varrho \in \mathbb{F}$. One checks that $\left\{\theta_{i}\right\}_{i=0}^{d}$ is $(\beta, \gamma, \varrho)$-recurrent. By Lemma 15.2 the scalars $\beta, \gamma, \varrho$ satisfy (TD1). Replacing $\Phi$ by $\Phi^{*}$ in the above argument, we obtain $\gamma^{*}, \varrho^{*} \in \mathbb{F}$ such that $\beta, \gamma^{*}, \varrho^{*}$ satisfy (TD2).

We emphasize one aspect of the above proof.

Corollary 15.6 (See [18, Lemma 12.7]). For the Leonard system $\Phi$ the scalars

$$
\frac{\theta_{i-2}-\theta_{i+1}}{\theta_{i-1}-\theta_{i}}, \quad \frac{\theta_{i-2}^{*}-\theta_{i+1}^{*}}{\theta_{i-1}^{*}-\theta_{i}^{*}}
$$

are equal and independent of $i$ for $2 \leq i \leq d-1$.

\section{The Tridiagonal Relations, Cont.}

Throughout this section let $\left\{\theta_{i}\right\}_{i=0}^{d},\left\{\theta_{i}^{*}\right\}_{i=0}^{d},\left\{\varphi_{i}\right\}_{i=1}^{d}$ denote scalars in $\mathbb{F}$. Define matrices $A, A^{*} \in \operatorname{Mat}_{d+1}(\mathbb{F})$ by 


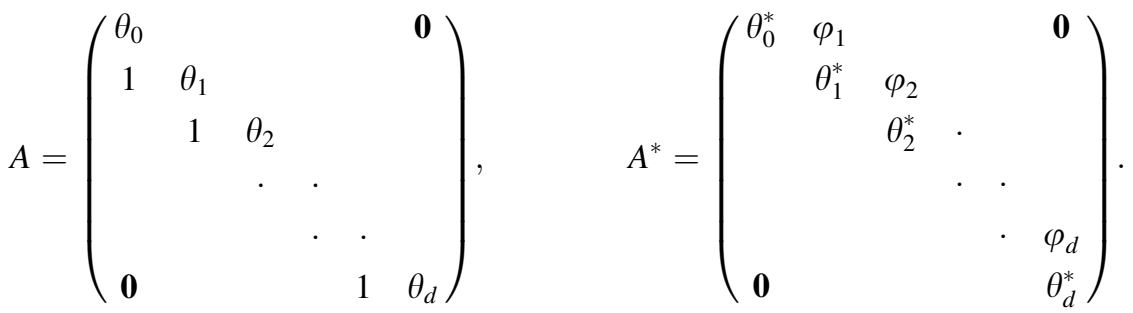

Definition 16.1 Define the scalars

$$
\vartheta_{i}=\varphi_{i}-\left(\theta_{i}^{*}-\theta_{0}^{*}\right)\left(\theta_{i-1}-\theta_{d}\right) \quad(1 \leq i \leq d)
$$

and $\vartheta_{0}=0, \vartheta_{d+1}=0$.

Lemma 16.2 (See [18, Lemma 12.4]). Let $\beta, \gamma, \varrho$ denote scalars in $\mathbb{F}$, and consider the commutator

$$
\left[A, A^{2} A^{*}-\beta A A^{*} A+A^{*} A^{2}-\gamma\left(A A^{*}+A^{*} A\right)-\varrho A^{*}\right] .
$$

Then the entries of (54) are as follows.

(i) The $(i+1, i-2)$-entry is

$$
\theta_{i-2}^{*}-(\beta+1) \theta_{i-1}^{*}+(\beta+1) \theta_{i}^{*}-\theta_{i+1}^{*}
$$

for $2 \leq i \leq d-1$.

(ii) The $(i, i-2)$-entry is

$$
\begin{aligned}
& \vartheta_{i-2}-(\beta+1) \vartheta_{i-1}+(\beta+1) \vartheta_{i}-\vartheta_{i+1} \\
& \quad+\left(\theta_{i-2}^{*}-\theta_{0}^{*}\right)\left(\theta_{i-3}-(\beta+1) \theta_{i-2}+(\beta+1) \theta_{i-1}-\theta_{i}\right) \\
& \quad+\left(\theta_{i}-\theta_{d}\right)\left(\theta_{i-2}^{*}-(\beta+1) \theta_{i-1}^{*}+(\beta+1) \theta_{i}^{*}-\theta_{i+1}^{*}\right) \\
& \quad+\left(\theta_{i-2}^{*}-\theta_{i}^{*}\right)\left(\theta_{i-2}-\beta \theta_{i-1}+\theta_{i}-\gamma\right)
\end{aligned}
$$

for $2 \leq i \leq d$, where $\left\{\vartheta_{i}\right\}_{i=0}^{d+1}$ are from Definition 16.1.

(iii) The $(i, i-1)$-entry is

$$
\begin{gathered}
\varphi_{i-1}\left(\theta_{i-2}-\beta \theta_{i-1}+\theta_{i}-\gamma\right)-\varphi_{i+1}\left(\theta_{i-1}-\beta \theta_{i}+\theta_{i+1}-\gamma\right) \\
+\left(\theta_{i-1}^{*}-\theta_{i}^{*}\right)\left(\theta_{i-1}^{2}-\beta \theta_{i-1} \theta_{i}+\theta_{i}^{2}-\gamma\left(\theta_{i-1}+\theta_{i}\right)-\varrho\right)
\end{gathered}
$$

for $1 \leq i \leq d$.

(iv) The $(i, i)$-entry is

$$
\begin{aligned}
& \varphi_{i}\left(\theta_{i-1}^{2}-\beta \theta_{i-1} \theta_{i}+\theta_{i}^{2}-\gamma\left(\theta_{i-1}+\theta_{i}\right)-\varrho\right) \\
& \quad-\varphi_{i+1}\left(\theta_{i}^{2}-\beta \theta_{i} \theta_{i+1}+\theta_{i+1}^{2}-\gamma\left(\theta_{i}+\theta_{i+1}\right)-\varrho\right)
\end{aligned}
$$

for $0 \leq i \leq d$.

(v) The $(i-1, i)$-entry is 


$$
\varphi_{i}\left(\theta_{i-1}-\theta_{i}\right)\left(\theta_{i-1}^{2}-\beta \theta_{i-1} \theta_{i}+\theta_{i}^{2}-\gamma\left(\theta_{i-1}+\theta_{i}\right)-\varrho\right)
$$

for $1 \leq i \leq d$.

All other entries in (54) are zero. In the above formulas, we assume $\varphi_{0}=0$, $\varphi_{d+1}=0$, and that $\theta_{-1}, \theta_{d+1}, \theta_{d+1}^{*}$ are indeterminates.

Proof Routine matrix multiplication.

Lemma 16.3 Let $\beta, \gamma, \varrho$ denote scalars in $\mathbb{F}$. Assume that $\left\{\theta_{i}\right\}_{i=0}^{d}$ are mutually distinct and $(\beta, \gamma, \varrho)$-recurrent. Assume that $\left\{\theta_{i}^{*}\right\}_{i=0}^{d}$ is $\beta$-recurrent. Then for the commutator (54) the $(i, i-2)$-entry is

$$
\vartheta_{i-2}-(\beta+1) \vartheta_{i-1}+(\beta+1) \vartheta_{i}-\vartheta_{i+1}
$$

for $2 \leq i \leq d$, where $\left\{\vartheta_{i}\right\}_{i=0}^{d+1}$ are from Definition16.1. All other entries in (54) are zero.

Proof Examine the entries given in Lemma 16.2.

Proposition 16.4 With the notation and assumptions of Lemma 16.3,

$$
0=\left[A, A^{2} A^{*}-\beta A A^{*} A+A^{*} A^{2}-\gamma\left(A A^{*}+A^{*} A\right)-\varrho A^{*}\right]
$$

if and only if $\left\{\vartheta_{i}\right\}_{i=0}^{d+1}$ is $\beta$-recurrent.

Proof By Lemma 16.3.

\section{The Proof of Theorem 10.1}

In this section we prove Theorem 10.1.

Proof of Theorem 10.1 We may assume that $d \geq 1$; otherwise the result is vacuous. Assume that there exists a Leonard system $\Phi=\left(A ;\left\{E_{i}\right\}_{i=0}^{d} ; A^{*} ;\left\{E_{i}^{*}\right\}_{i=0}^{d}\right)$ over $\mathbb{F}$ with parameter array (21). We show that this parameter array satisfies (PA1)-(PA5). Condition (PA1) holds since $A$ and $A^{*}$ are multiplicity-free. Condition (PA2) holds by the comment below Definition 9.1. Condition (PA5) holds by Corollary 15.6. By (PA5) and Lemma 11.2, there exists $\beta \in \mathbb{F}$ such that $\left\{\theta_{i}\right\}_{i=0}^{d}$ is $\beta$-recurrent and $\left\{\theta_{i}^{*}\right\}_{i=0}^{d}$ is $\beta$-recurrent. Concerning (PA3), Define

$$
\vartheta_{i}=\varphi_{i}-\left(\theta_{i}^{*}-\theta_{0}^{*}\right)\left(\theta_{i-1}-\theta_{d}\right) \quad(1 \leq i \leq d) .
$$

We show that

$$
\vartheta_{i}=\phi_{1} \sum_{h=0}^{i-1} \frac{\theta_{h}-\theta_{d-h}}{\theta_{0}-\theta_{d}} \quad(1 \leq i \leq d) .
$$

To this end we invoke Proposition 13.4. We will show (i) $\vartheta_{1}=\phi_{1}$; (ii) $\vartheta_{d}=\phi_{1}$; 
(iii) $\left\{\vartheta_{i}\right\}_{i=0}^{d+1}$ is $\beta$-recurrent, where $\vartheta_{0}=0$ and $\vartheta_{d+1}=0$. To show (i), we compare the formulas for $a_{0}$ in Lemmas 8.1, 9.4 to obtain $\varphi_{1}-\phi_{1}=\left(\theta_{1}^{*}-\theta_{0}^{*}\right)\left(\theta_{0}-\theta_{d}\right)$. We have

$$
\vartheta_{1}=\varphi_{1}-\left(\theta_{1}^{*}-\theta_{0}^{*}\right)\left(\theta_{0}-\theta_{d}\right)=\phi_{1} .
$$

To show (ii), we compare the formulas for $a_{d}^{*}$ in Lemmas 8.1, 9.4 to obtain $\varphi_{d}-\phi_{1}=\left(\theta_{d}^{*}-\theta_{0}^{*}\right)\left(\theta_{d-1}-\theta_{d}\right)$. We have

$$
\vartheta_{d}=\varphi_{d}-\left(\theta_{d}^{*}-\theta_{0}^{*}\right)\left(\theta_{d-1}-\theta_{d}\right)=\phi_{1} .
$$

To show (iii) we apply Proposition 16.4 to the matrix representations of $A, A^{*}$ from Proposition 7.6(iii). Recall that $\left\{\theta_{i}\right\}_{i=0}^{d}$ is $\beta$-recurrent. By Lemma 11.3 there exists $\gamma \in \mathbb{F}$ such that $\left\{\theta_{i}\right\}_{i=0}^{d}$ is $(\beta, \gamma)$-recurrent. By Lemma 11.4(i) there exists $\varrho \in \mathbb{F}$ such that $\left\{\theta_{i}\right\}_{i=0}^{d}$ is $(\beta, \gamma, \varrho)$-recurrent. The scalars $\beta, \gamma, \varrho$ satisfy (TD1) by Lemma 15.2. The assumptions of Lemma 16.3 are satisfied, so by Proposition 16.4 the sequence $\left\{\vartheta_{i}\right\}_{i=0}^{d+1}$ is $\beta$-recurrent. We have shown (i)-(iii). Now (55) holds by Proposition 13.4, so (PA3) holds. To obtain (PA4), apply (PA3) to the Leonard system $\Phi^{\Downarrow}$, and use Lemma 9.7. We have obtained (PA1)-(PA5), and we are done in one direction.

We now reverse the direction. Assume that the scalars (21) satisfy (PA1)-(PA5). We display a Leonard system $\Phi$ over $\mathbb{F}$ that has parameter array (21). Recall the vector space $V$ with dimension $d+1$. Pick a basis $\left\{u_{i}\right\}_{i=0}^{d}$ for $V$. Define $A, A^{*} \in$ $\operatorname{End}(V)$ with the following matrix representations with respect to $\left\{u_{i}\right\}_{i=0}^{d}$ :

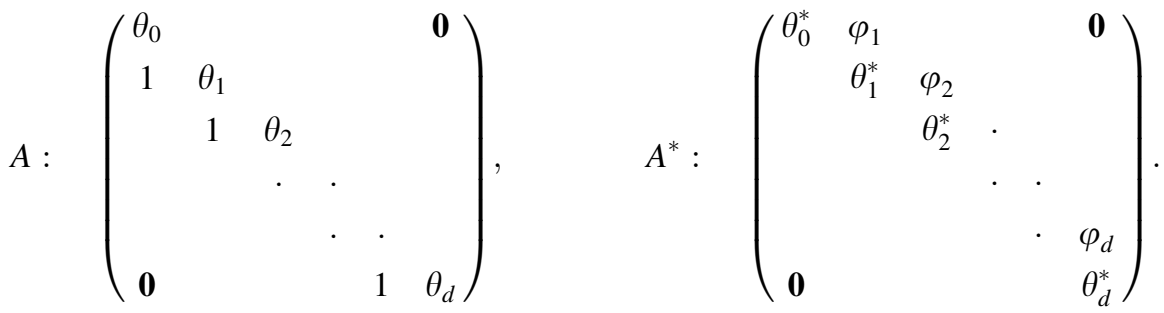

Observe that $A$ (resp. $A^{*}$ ) is multiplicity-free with eigenvalues $\left\{\theta_{i}\right\}_{i=0}^{d}$ (resp. $\left\{\theta_{i}^{*}\right\}_{i=0}^{d}$ ); for $0 \leq i \leq d$ let $E_{i}$ (resp. $E_{i}^{*}$ ) denote the primitive idempotent of $A$ (resp. $\left.A^{*}\right)$ for $\theta_{i}\left(\right.$ resp. $\left.\theta_{i}^{*}\right)$. The sequence $\Phi:=\left(A ;\left\{E_{i}\right\}_{i=0}^{d} ; A^{*} ;\left\{E_{i}^{*}\right\}_{i=0}^{d}\right)$ is a pre Leonard system on $V$. We show that $\Phi$ is a Leonard system on $V$. To this end we show the following (56)-(64) for $0 \leq i, j \leq d$ :

$$
\begin{array}{ll}
E_{i}^{*} A E_{j}^{*}=0 \quad \text { if } & i-j>1 ; \\
E_{i}^{*} A E_{j}^{*}=0 \quad \text { if } & j-i>1 ; \\
E_{i}^{*} A E_{j}^{*} \neq 0 \quad \text { if } \quad i-j=1 ;
\end{array}
$$




$$
E_{i}^{*} A E_{j}^{*} \neq 0 \quad \text { if } \quad j-i=1
$$

and

$$
\begin{gathered}
E_{i} A^{*} E_{j}=0 \quad \text { if } \quad d>i-j>1 \\
E_{i} A^{*} E_{j}=0 \quad \text { if } \quad d=i-j>1 \\
E_{i} A^{*} E_{j}=0 \quad \text { if } \quad j-i>1 \\
E_{i} A^{*} E_{j} \neq 0 \quad \text { if } \quad i-j=1 \\
E_{i} A^{*} E_{j} \neq 0 \quad \text { if } \quad j-i=1 .
\end{gathered}
$$

Proposition 7.6 implies (56), (58), (62). Lemma 7.7 implies (64). Before proceeding we make some comments. The element $E_{0}^{*}$ is normalizing by Proposition 7.6. By (PA5) and Lemma 11.2, there exists $\beta \in \mathbb{F}$ such that $\left\{\theta_{i}\right\}_{i=0}^{d}$ is $\beta$-recurrent and $\left\{\theta_{i}^{*}\right\}_{i=0}^{d}$ is $\beta$-recurrent. By Lemma 11.3 there exists $\gamma \in \mathbb{F}$ such that $\left\{\theta_{i}\right\}_{i=0}^{d}$ is $(\beta, \gamma)$ recurrent. By Lemma 11.4(i) there exists $\varrho \in \mathbb{F}$ such that $\left\{\theta_{i}\right\}_{i=0}^{d}$ is $(\beta, \gamma, \varrho)$-recurrent. By (PA3), for $1 \leq i \leq d$ we have

$$
\varphi_{i}-\left(\theta_{i}^{*}-\theta_{0}^{*}\right)\left(\theta_{i-1}-\theta_{d}\right)=\phi_{1} \sum_{h=0}^{i-1} \frac{\theta_{h}-\theta_{d-h}}{\theta_{0}-\theta_{d}}
$$

let $\vartheta_{i}$ denote this common value. Note that $\vartheta_{1}=\phi_{1}=\vartheta_{d}$. For notational convenience define $\vartheta_{0}=0$ and $\vartheta_{d+1}=0$.

We show (60). The sequence $\left\{\vartheta_{i}\right\}_{i=0}^{d+1}$ satisfies Proposition 13.4(i). By Proposition 13.4 the sequence $\left\{\vartheta_{i}\right\}_{i=0}^{d+1}$ is $\beta$-recurrent. Next we apply Proposition 16.4. We mentioned earlier that $\left\{\theta_{i}\right\}_{i=0}^{d}$ is $(\beta, \gamma, \varrho)$-recurrent and $\left\{\theta_{i}^{*}\right\}_{i=0}^{d}$ is $\beta$-recurrent. By these comments and Proposition 16.4, the scalars $\beta, \gamma, \varrho$ satisfy (TD1). For $0 \leq i, j \leq d$ we multiply each term in (TD1) on the left by $E_{i}$ and on the right by $E_{j}$. This yields

$$
0=E_{i} A^{*} E_{j}\left(\theta_{i}-\theta_{j}\right) P\left(\theta_{i}, \theta_{j}\right),
$$

where $P$ is from (46). By Proposition 14.2(ii) we obtain $P\left(\theta_{i}, \theta_{j}\right) \neq 0$ if $d>i-j>1$. By this and (65) we have $E_{i} A^{*} E_{j}=0$ if $d>i-j>1$. We have shown (60).

We show (61). We may assume $d \geq 2$; otherwise there is nothing to prove. We show $E_{d} A^{*} E_{0}=0$. Since $E_{0}^{*}$ is normalizing, it suffices to show that $E_{d} A^{*} E_{0} E_{0}^{*}=0$ in view of Proposition 6.4. To show that $E_{d} A^{*} E_{0} E_{0}^{*}=0$, we invoke Proposition 8.4. By (60) we have $E_{d} A^{*} E_{i}=0$ for $1 \leq i \leq d-2$. We mentioned earlier that $\vartheta_{1}=\vartheta_{d}$. These comments and Proposition 8.4 imply $E_{d} A^{*} E_{0} E_{0}^{*}\left(\theta_{0}-\theta_{d-1}\right)=0$. The scalar $\theta_{0}-\theta_{d-1}$ is nonzero since $d \geq 2$, so $E_{d} A^{*} E_{0} E_{0}^{*}=0$. We have shown (61).

We show (63). We must show that $E_{i} A^{*} E_{i-1} \neq 0$ for $1 \leq i \leq d$. To this end we first apply Proposition 7.6 to $\Phi^{\Downarrow}$. Proposition 7.6(i) holds for $\Phi^{\Downarrow}$ by (56), (58), (60), 
(61). So Proposition 7.6(iii) holds for $\Phi^{\Downarrow}$. Consequently there exist scalars $\left\{\varphi_{i}^{\Downarrow}\right\}_{i=1}^{d}$ in $\mathbb{F}$ and a basis for $V$ with respect to which

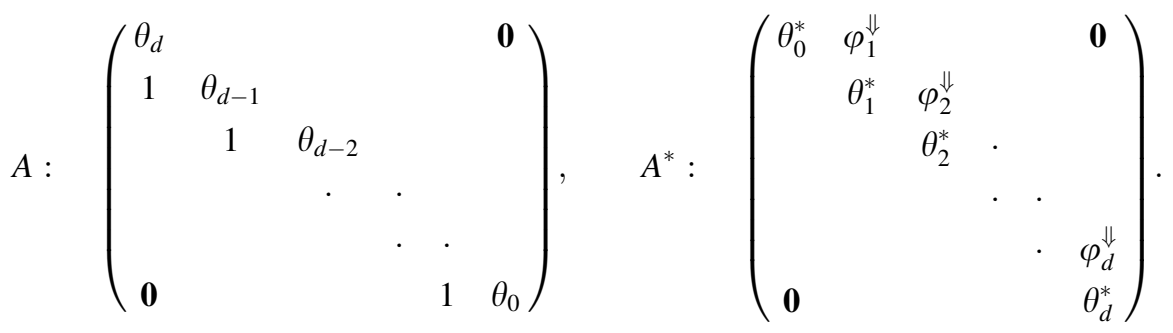

We are trying to show that $E_{i} A^{*} E_{i-1} \neq 0$ for $1 \leq i \leq d$. By Lemma 7.7 (applied to $\left.\Phi^{\Downarrow}\right)$, it suffices to show that $\varphi_{i}^{\Downarrow} \neq 0$ for $1 \leq i \leq d$. To this end we show that $\varphi_{i}^{\Downarrow}=\phi_{i}$ for $1 \leq i \leq d$. By (PA4),

$$
\phi_{i}-\left(\theta_{i}^{*}-\theta_{0}^{*}\right)\left(\theta_{d-i+1}-\theta_{0}\right)=\varphi_{1} \sum_{h=0}^{i-1} \frac{\theta_{h}-\theta_{d-h}}{\theta_{0}-\theta_{d}} \quad(1 \leq i \leq d) .
$$

Define

$$
\vartheta_{i}^{\Downarrow}=\varphi_{i}^{\Downarrow}-\left(\theta_{i}^{*}-\theta_{0}^{*}\right)\left(\theta_{d-i+1}-\theta_{0}\right) \quad(1 \leq i \leq d) .
$$

We show

$$
\vartheta_{i}^{\Downarrow}=\varphi_{1} \sum_{h=0}^{i-1} \frac{\theta_{h}-\theta_{d-h}}{\theta_{0}-\theta_{d}} \quad(1 \leq i \leq d) .
$$

To show (67), by Proposition 13.4 it suffices to show (i) $\vartheta_{1}^{\Downarrow}=\varphi_{1}$; (ii) $\vartheta_{1}^{\Downarrow}=\vartheta_{d}^{\Downarrow}$; (iii) $\left\{\vartheta_{i}^{\Downarrow}\right\}_{i=0}^{d+1}$ is $\beta$-recurrent, where $\vartheta_{0}^{\Downarrow}=0$ and $\vartheta_{d+1}^{\Downarrow}=0$. To show (i), for $\Phi$ and $\Phi^{\Downarrow}$ we compute $a_{0}$ using Lemma 8.1; this yields $a_{0}=\theta_{0}+\varphi_{1}\left(\theta_{0}^{*}-\theta_{1}^{*}\right)^{-1}$ and $a_{0}=\theta_{d}+\varphi_{1}^{\Downarrow}\left(\theta_{0}^{*}-\theta_{1}^{*}\right)^{-1}$. Combining these equations we obtain $\varphi_{1}-\varphi_{1}^{\Downarrow}=\left(\theta_{1}^{*}-\theta_{0}^{*}\right)\left(\theta_{0}-\theta_{d}\right)$, so

$$
\vartheta_{1}^{\Downarrow}=\varphi_{1}^{\Downarrow}-\left(\theta_{1}^{*}-\theta_{0}^{*}\right)\left(\theta_{d}-\theta_{0}\right)=\varphi_{1} .
$$

To show (ii), we apply Proposition 8.4 to $\Phi^{\Downarrow}$. This gives

$$
\sum_{i=0}^{d-2} E_{0} A^{*} E_{d-i} E_{0}^{*}\left(\theta_{d-i}-\theta_{1}\right)=E_{0} E_{0}^{*}\left(\vartheta_{1}^{\Downarrow}-\vartheta_{d}^{\Downarrow}\right) .
$$

For this equation the left-hand side is zero, since each summand is zero by (62). We mentioned earlier that $E_{0}^{*}$ is normalizing, so $E_{0} E_{0}^{*} \neq 0$ by Definition 6.1. By these comments $\vartheta_{1}^{\Downarrow}=\vartheta_{d}^{\Downarrow}$. To show (iii), we apply Proposition 16.4 to the matrices (66). Recall that $\left\{\theta_{i}\right\}_{i=0}^{d}$ is $(\beta, \gamma, \varrho)$-recurrent and $\left\{\theta_{i}^{*}\right\}_{i=0}^{d}$ is $\beta$-recurrent. We mentioned 
earlier that $\beta, \gamma, \varrho$ satisfy (TD1). Applying Proposition 16.4 to the matrices (66), we see that $\left\{\vartheta_{i}^{\Downarrow}\right\}_{i=0}^{d+1}$ is $\beta$-recurrent. We have shown (i)-(iii), so (67) holds by Proposition 13.4. Now by the construction $\varphi_{i}^{\Downarrow}=\phi_{i}$ for $1 \leq i \leq d$. Consequently $\varphi_{i}^{\Downarrow} \neq 0$ for $1 \leq i \leq d$, so $E_{i} A^{*} E_{i-1} \neq 0$ for $1 \leq i \leq d$. We have shown (63).

We show (57) and (59) by invoking Proposition 4.4. Consider the conditions (i)(iv) in that proposition. Condition (i) holds by (56), (58). Condition (iii) holds by (60), (61), (63). Condition (iv) holds by (62), (64). Condition (ii) holds by Proposition 4.4, and this implies (57) and (59).

We have shown (56)-(64), so $\Phi$ is a Leonard system on $V$. By construction $\Phi$ has eigenvalue sequence $\left\{\theta_{i}\right\}_{i=0}^{d}$, dual eigenvalue sequence $\left\{\theta_{i}^{*}\right\}_{i=0}^{d}$, and first split sequence $\left\{\varphi_{i}\right\}_{i=1}^{d}$. By construction $\left\{\varphi_{i}^{\Downarrow}\right\}_{i=1}^{d}$ is the first split sequence of $\Phi^{\Downarrow}$ and hence the second split sequence of $\Phi$. We showed $\varphi_{i}^{\Downarrow}=\phi_{i}$ for $1 \leq i \leq d$, so $\left\{\phi_{i}\right\}_{i=1}^{d}$ is the second split sequence for $\Phi$. By these comments and Definition 9.6, the sequence (21) is the parameter array of $\Phi$. By Proposition 9.8 the Leonard system $\Phi$ is unique up to isomorphism.

\section{Characterizations of Leonard Systems and Parameter Arrays}

We are done discussing Theorem 10.1. In this section we discuss some related results concerning Leonard systems and parameter arrays.

We comment on notation. Let $\left\{u_{i}\right\}_{i=0}^{d}$ and $\left\{v_{i}\right\}_{i=0}^{d}$ denote bases for $V$. By the transition matrix from $\left\{u_{i}\right\}_{i=0}^{d}$ to $\left\{v_{i}\right\}_{i=0}^{d}$ we mean the matrix $M \in \operatorname{Mat}_{d+1}(\mathbb{F})$ such that $v_{j}=\sum_{i=0}^{d} M_{i j} u_{i}$ for $0 \leq j \leq d$.

The following result is a variation on [22, Theorem 5.1].

Theorem 18.1 Let $\Phi=\left(A ;\left\{E_{i}\right\}_{i=0}^{d} ; A^{*} ;\left\{E_{i}^{*}\right\}_{i=0}^{d}\right)$ denote a pre Leonard system on $V$, with eigenvalue sequence $\left\{\theta_{i}\right\}_{i=0}^{d}$ and dual eigenvalue sequence $\left\{\theta_{i}^{*}\right\}_{i=0}^{d}$. Then $\Phi$ is a Leonard system on $V$ if and only if the following (i), (ii) hold:

(i) there exist nonzero scalars $\left\{\varphi_{i}\right\}_{i=1}^{d}$ in $\mathbb{F}$ and a basis for $V$ with respect to which

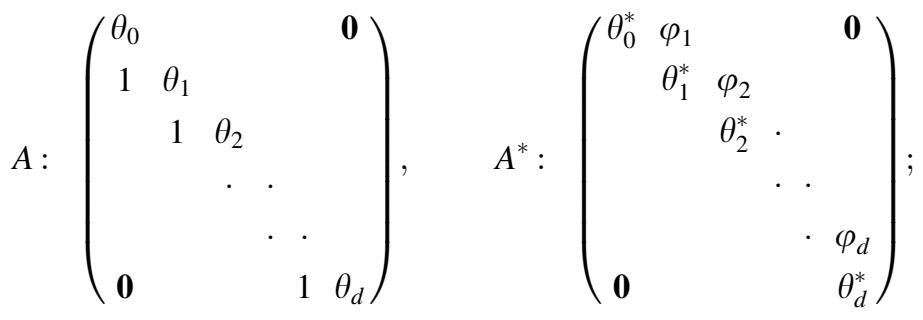

(ii) there exist nonzero scalars $\left\{\phi_{i}\right\}_{i=1}^{d}$ in $\mathbb{F}$ and a basis for $V$ with respect to which 


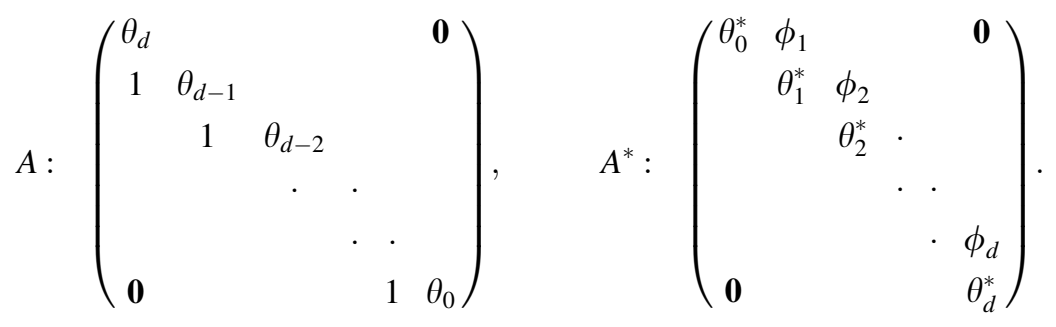

In this case $\left(\left\{\theta_{i}\right\}_{i=0}^{d} ;\left\{\theta_{i}^{*}\right\}_{i=0}^{d} ;\left\{\varphi_{i}\right\}_{i=1}^{d} ;\left\{\phi_{i}\right\}_{i=1}^{d}\right)$ is the parameter array of $\Phi$.

Proof Apply Proposition 7.6 and Lemma 7.7 to both $\Phi$ and $\Phi^{\Downarrow}$.

Using Theorem 18.1 we can easily recover the following result.

Theorem 18.2 (See [21, Theorem 3.2]). Consider a sequence

$$
\left(\left\{\theta_{i}\right\}_{i=0}^{d} ;\left\{\theta_{i}^{*}\right\}_{i=0}^{d} ;\left\{\varphi_{i}\right\}_{i=1}^{d} ;\left\{\phi_{i}\right\}_{i=1}^{d}\right)
$$

of scalars in $\mathbb{E}$ that satisfies (PA1), (PA2). Then the following are equivalent:

(i) the sequence (68) satisfies (PA3)-(PA5);

(ii) there exists an invertible $G \in \mathrm{Mat}_{d+1}(\mathbb{F})$ such that both

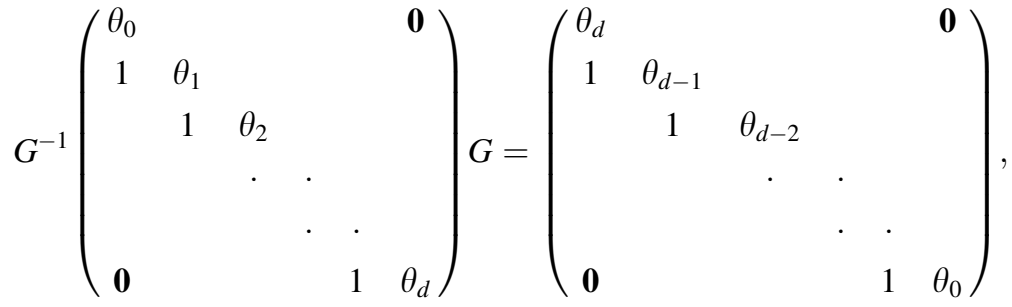

$$
\begin{aligned}
& G^{-1}\left(\begin{array}{cccccc}
\theta_{0}^{*} & \varphi_{1} & & & & \mathbf{0} \\
& \theta_{1}^{*} & \varphi_{2} & & & \\
& & \theta_{2}^{*} & . & & \\
& & & \cdot & \cdot & \\
& & & & \cdot & \varphi_{d} \\
& & & & & \theta_{d}^{*}
\end{array}\right) G=\left(\begin{array}{cccccc}
\theta_{0}^{*} & \phi_{1} & & & & \mathbf{0} \\
& \theta_{1}^{*} & \phi_{2} & & & \\
& & \theta_{2}^{*} & \cdot & & \\
& & & \cdot & \cdot & \\
& & & & \cdot & \phi_{d} \\
& & & & & \theta_{d}^{*}
\end{array}\right) .
\end{aligned}
$$

Proof (i) $\Rightarrow$ (ii) By Theorem 10.1 there exists a Leonard system on $V$ with parameter array (68). The matrix $G$ is the transition matrix from a basis for $V$ that satisfies Theorem 18.1(i), to a basis for $V$ that satisfies Theorem 18.1(ii).

(ii) $\Rightarrow$ (i) Pick a basis $\left\{u_{i}\right\}_{i=0}^{d}$ for $V$. Define $A, A^{*} \in \operatorname{End}(V)$ whose matrix representations with respect to $\left\{u_{i}\right\}_{i=0}^{d}$ are from Theorem 18.1(i). Observe that $A$ (resp. $\left.A^{*}\right)$ is multiplicity-free with eigenvalues $\left\{\theta_{i}\right\}_{i=0}^{d}$ (resp. $\left\{\theta_{i}^{*}\right\}_{i=0}^{d}$ ); for $0 \leq i \leq d$ let $E_{i}$ (resp. $E_{i}^{*}$ ) denote the primitive idempotent of $A$ (resp. $\left.A^{*}\right)$ for $\theta_{i}\left(\right.$ resp. $\theta_{i}^{*}$ ). The 
sequence $\Phi:=\left(A ;\left\{E_{i}\right\}_{i=0}^{d} ; A^{*} ;\left\{E_{i}^{*}\right\}_{i=0}^{d}\right)$ is a pre Leonard system on $V$. We show that $\Phi$ is a Leonard system on $V$. By linear algebra there exists a basis $\left\{v_{i}\right\}_{i=0}^{d}$ of $V$ such that $G$ is the transition matrix from $\left\{u_{i}\right\}_{i=0}^{d}$ to $\left\{v_{i}\right\}_{i=0}^{d}$. The matrix representations of $A$ and $A^{*}$ with respect to $\left\{v_{i}\right\}_{i=0}^{d}$ are shown in Theorem 18.1(ii). The pre Leonard system $\Phi$ satisfies Theorem 18.1(i) and Theorem 18.1(ii), so by that theorem $\Phi$ is a Leonard system on $V$. Also by that theorem (68) is the parameter array of $\Phi$, so (68) satisfies (PA3)-(PA5).

\section{The Intersection Numbers}

We now bring in the intersection numbers. Throughout this section $\Phi=$ $\left(A ;\left\{E_{i}\right\}_{i=0}^{d} ; A^{*} ;\left\{E_{i}^{*}\right\}_{i=0}^{d}\right)$ denotes a Leonard system on $V$, with parameter array $\left(\left\{\theta_{i}\right\}_{i=0}^{d} ;\left\{\theta_{i}^{*}\right\}_{i=0}^{d} ;\left\{\varphi_{i}\right\}_{i=1}^{d} ;\left\{\phi_{i}\right\}_{i=1}^{d}\right)$. Applying Lemma 7.5 to $\Phi^{*}$ we see that $E_{0}$ is normalizing. For $0 \neq \xi \in E_{0} V$ the vectors $\left\{E_{i}^{*} \xi\right\}_{i=0}^{d}$ form a basis for $V$, said to be $\Phi$ standard. With respect to this basis

$$
A: \quad\left(\begin{array}{cccccc}
a_{0} & b_{0} & & & & \text { 0 } \\
c_{1} & a_{1} & b_{1} & & & \\
& c_{2} & \cdot & . & & \\
& & \cdot & \cdot & \cdot & \\
& & & \cdot & \cdot & b_{d-1} \\
\mathbf{0} & & & & c_{d} & a_{d}
\end{array}\right), \quad A^{*}: \operatorname{diag}\left(\theta_{0}^{*}, \theta_{1}^{*}, \ldots, \theta_{d}^{*}\right),
$$

where $\left\{a_{i}\right\}_{i=0}^{d}$ are from Definition 3.3 and $\left\{b_{i}\right\}_{i=0}^{d-1},\left\{c_{i}\right\}_{i=1}^{d}$ are nonzero scalars in $\mathbb{F}$. The vector $\xi$ is an eigenvector for $A$ with eigenvalue $\theta_{0}$. Moreover $\xi=\sum_{i=0}^{d} E_{i}^{*} \xi$. Consequently

$$
c_{i}+a_{i}+b_{i}=\theta_{0} \quad(0 \leq i \leq d),
$$

where $c_{0}=0$ and $b_{d}=0$. We call $\left\{b_{i}\right\}_{i=0}^{d-1},\left\{c_{i}\right\}_{i=1}^{d}$ the intersection numbers of $\Phi$. They are discussed in [20, Sect. 11]. The intersection numbers $\left\{b_{i}^{*}\right\}_{i=0}^{d-1},\left\{c_{i}^{*}\right\}_{i=1}^{d}$ of $\Phi^{*}$ are called the dual intersection numbers of $\Phi$. By construction

$$
c_{i}^{*}+a_{i}^{*}+b_{i}^{*}=\theta_{0}^{*} \quad(0 \leq i \leq d),
$$

where $c_{0}^{*}=0$ and $b_{d}^{*}=0$. With respect to a $\Phi^{*}$-standard basis for $V$, 


$$
A^{*}:\left(\begin{array}{cccccc}
a_{0}^{*} & b_{0}^{*} & & & & \mathbf{0} \\
c_{1}^{*} & a_{1}^{*} & b_{1}^{*} & & & \\
& c_{2}^{*} & \cdot & \cdot & & \\
& & \cdot & \cdot & \cdot & \\
& & & \cdot & \cdot & b_{d-1}^{*} \\
\mathbf{0} & & & & c_{d}^{*} & a_{d}^{*}
\end{array}\right) .
$$

We mention a handy recurrence.

Lemma 19.1 Assume $d \geq 1$. Then for $0 \leq i \leq d$ we have

$$
\begin{aligned}
& c_{i} \theta_{i-1}^{*}+a_{i} \theta_{i}^{*}+b_{i} \theta_{i+1}^{*}=\theta_{1} \theta_{i}^{*}+a_{0}^{*}\left(\theta_{0}-\theta_{1}\right), \\
& c_{i}^{*} \theta_{i-1}+a_{i}^{*} \theta_{i}+b_{i}^{*} \theta_{i+1}=\theta_{1}^{*} \theta_{i}+a_{0}\left(\theta_{0}^{*}-\theta_{1}^{*}\right),
\end{aligned}
$$

where $\theta_{-1}, \theta_{d+1}, \theta_{-1}^{*}, \theta_{d+1}^{*}$ denote indeterminates.

Proof The proof of (72) is similar to the proof of (69). To obtain (72), use the fact that for $0 \neq \xi \in E_{0} V$ the vector $\left(A^{*}-a_{0}^{*} I\right) \xi$ is an eigenvector for $A$ with eigenvalue $\theta_{1}$, and $\left(A^{*}-a_{0}^{*} I\right) \xi=\sum_{i=0}^{d}\left(\theta_{i}^{*}-a_{0}^{*}\right) E_{i}^{*} \xi$. To get (73), apply (72) to $\Phi^{*}$.

Lemma 19.2 For $d \geq 1$ we have

$$
\begin{array}{ll}
b_{0}=\theta_{0}-a_{0}, \\
b_{i}=\frac{\left(a_{i}-\theta_{0}\right)\left(\theta_{i}^{*}-\theta_{i-1}^{*}\right)+\left(\theta_{0}-\theta_{1}\right)\left(\theta_{i}^{*}-a_{0}^{*}\right)}{\theta_{i-1}^{*}-\theta_{i+1}^{*}} \quad(1 \leq i \leq d-1), \\
c_{i}=\frac{\left(a_{i}-\theta_{0}\right)\left(\theta_{i}^{*}-\theta_{i+1}^{*}\right)+\left(\theta_{0}-\theta_{1}\right)\left(\theta_{i}^{*}-a_{0}^{*}\right)}{\theta_{i+1}^{*}-\theta_{i-1}^{*}} \quad(1 \leq i \leq d-1), \\
c_{d}=\theta_{0}-a_{d} .
\end{array}
$$

To get $\left\{b_{i}^{*}\right\}_{i=0}^{d-1}$ and $\left\{c_{i}^{*}\right\}_{i=1}^{d}$, exchange starred and nonstarred symbols everywhere in the above equations.

Proof To obtain $b_{0}$ set $i=0$ in (69). To obtain $b_{i}, c_{i}$ for $1 \leq i \leq d-1$, solve the linear equations (69), (72). To obtain $c_{d}$ set $i=d$ in (69). To obtain $\left\{b_{i}^{*}\right\}_{i=0}^{d-1}$ and $\left\{c_{i}^{*}\right\}_{i=1}^{d}$, apply the above comments to $\Phi^{*}$.

In Lemma 19.1 we gave a recurrence. We now give a more general recurrence.

Lemma 19.3 For $0 \leq i \leq d$ and $1 \leq j \leq d$,

$$
\begin{gathered}
c_{i} \tau_{j}^{*}\left(\theta_{i-1}^{*}\right)+a_{i} \tau_{j}^{*}\left(\theta_{i}^{*}\right)+b_{i} \tau_{j}^{*}\left(\theta_{i+1}^{*}\right)=\theta_{j} \tau_{j}^{*}\left(\theta_{i}^{*}\right)+\varphi_{j} \tau_{j-1}^{*}\left(\theta_{i}^{*}\right), \\
c_{i} \eta_{j}^{*}\left(\theta_{i-1}^{*}\right)+a_{i} \eta_{j}^{*}\left(\theta_{i}^{*}\right)+b_{i} \eta_{j}^{*}\left(\theta_{i+1}^{*}\right)=\theta_{j} \eta_{j}^{*}\left(\theta_{i}^{*}\right)+\phi_{d-j+1} \eta_{j-1}^{*}\left(\theta_{i}^{*}\right),
\end{gathered}
$$




$$
\begin{aligned}
& c_{i}^{*} \tau_{j}\left(\theta_{i-1}\right)+a_{i}^{*} \tau_{j}\left(\theta_{i}\right)+b_{i}^{*} \tau_{j}\left(\theta_{i+1}\right)=\theta_{j}^{*} \tau_{j}\left(\theta_{i}\right)+\varphi_{j} \tau_{j-1}\left(\theta_{i}\right), \\
& c_{i}^{*} \eta_{j}\left(\theta_{i-1}\right)+a_{i}^{*} \eta_{j}\left(\theta_{i}\right)+b_{i}^{*} \eta_{j}\left(\theta_{i+1}\right)=\theta_{j}^{*} \eta_{j}\left(\theta_{i}\right)+\phi_{j} \eta_{j-1}\left(\theta_{i}\right),
\end{aligned}
$$

where $\theta_{-1}, \theta_{d+1}, \theta_{-1}^{*}, \theta_{d+1}^{*}$ denote indeterminates.

Proof Concerning (76), Let $T \in \operatorname{Mat}_{d+1}(\mathbb{F})$ have $(i, j)$-entry $\tau_{j}\left(\theta_{i}\right)$ for $0 \leq i, j \leq d$. For $0 \neq \xi \in E_{0}^{*} V, T$ is the transition matrix from the $\Phi^{*}$-standard basis $\left\{E_{i} \xi\right\}_{i=0}^{d}$ to the basis $\left\{\tau_{i}(A) \xi\right\}_{i=0}^{d}$. The matrix $B^{*}$ on the right in (71) represents $A^{*}$ with respect to $\left\{E_{i} \xi\right\}_{i=0}^{d}$. The matrix $D^{*}$ on the right in (12) represents $A^{*}$ with respect to $\left\{\tau_{i}(A) \xi\right\}_{i=0}^{d}$. By linear algebra $B^{*} T=T D^{*}$. The entries of this matrix give the equations (76). To obtain (77), in the above argument replace the basis $\left\{\tau_{i}(A) \xi\right\}_{i=0}^{d}$ by the basis $\left\{\eta_{i}(A) \xi\right\}_{i=0}^{d}$, and replace the matrix on the right in (12) by the matrix on the right in (20). To obtain (74) and (75), apply (76) and (77) to $\Phi^{*}$.

Lemma 19.4 (See [20, Theorem 17.7]). We have
(i) $\quad b_{i}=\varphi_{i+1} \frac{\tau_{i}^{*}\left(\theta_{i}^{*}\right)}{\tau_{i+1}^{*}\left(\theta_{i+1}^{*}\right)} \quad(0 \leq i \leq d-1)$;
(ii) $\quad c_{i}=\phi_{i} \frac{\eta_{d-i}^{*}\left(\theta_{i}^{*}\right)}{\eta_{d-i+1}^{*}\left(\theta_{i-1}^{*}\right)} \quad(1 \leq i \leq d)$;
(iii) $\quad b_{i}^{*}=\varphi_{i+1} \frac{\tau_{i}\left(\theta_{i}\right)}{\tau_{i+1}\left(\theta_{i+1}\right)} \quad(0 \leq i \leq d-1)$;
(iv) $\quad c_{i}^{*}=\phi_{d-i+1} \frac{\eta_{d-i}\left(\theta_{i}\right)}{\eta_{d-i+1}\left(\theta_{i-1}\right)} \quad(1 \leq i \leq d)$.

\section{Proof}

(i) Set $j=i+1$ in (74).

(ii) Set $j=d-i+1$ in (75).

(iii) $\operatorname{Set} j=i+1$ in (76).

(ii) Set $j=d-i+1$ in (77).

In Lemma 19.4 we see some fractions. In order to simplify these fractions, we consider the products

$$
\psi_{i}=\prod_{h=0}^{i-2} \frac{\theta_{i}-\theta_{h+1}}{\theta_{i+1}-\theta_{h}} \quad(1 \leq i \leq d-1) .
$$

Note that $\psi_{1}=1$. Using Definition 5.1 we find that for $1 \leq i \leq d-1$,

$$
\frac{\tau_{i}\left(\theta_{i}\right)}{\tau_{i+1}\left(\theta_{i+1}\right)}=\frac{\psi_{i}\left(\theta_{i}-\theta_{0}\right)}{\left(\theta_{i+1}-\theta_{i}\right)\left(\theta_{i+1}-\theta_{i-1}\right)} .
$$

We now describe the scalars $\left\{\psi_{i}\right\}_{i=1}^{d-1}$ in detail. To avoid trivialities we assume that $d \geq 3$. Let $\beta+1$ denote the common value of (22).

Lemma 19.5 For $d \geq 3$ and $1 \leq i \leq d-1$ we have the following. 
(i) Suppose $\beta \neq 2, \beta \neq-2$. Then

$$
\psi_{i}=q^{i-1} \frac{q-1}{q^{i}-1} \frac{q^{2}-1}{q^{i+1}-1},
$$

where $q+q^{-1}=\beta$.

(ii) Suppose $\beta=2$ and $\operatorname{char}(\mathbb{F}) \neq 2$. Then

$$
\psi_{i}=\frac{2}{i(i+1)} .
$$

(iii) Suppose $\beta=-2$ and $\operatorname{char}(\mathbb{F}) \neq 2$. Then

$$
\psi_{i}=\left\{\begin{array}{cc}
-2 / i, & \text { if } i \text { is even } \\
2 /(i+1), & \text { if } i \text { is odd }
\end{array}\right.
$$

(iv) Suppose $\beta=0$, $\operatorname{char}(\mathbb{F})=2$, and $d=3$. Then

$$
\psi_{i}=1
$$

Proof Evaluate the right-hand side of (78) using Lemma 12.3, and simplify the result.

Note 19.6 Under the assumptions of Lemma 19.5(iii),

$$
\psi_{i}=\frac{4(-1)^{i}}{(-1)^{i}-1-2 i} \quad(1 \leq i \leq d-1) .
$$

Corollary 19.7 For $1 \leq i \leq d-1$,

$$
\begin{aligned}
\frac{\tau_{i}^{*}\left(\theta_{i}^{*}\right)}{\tau_{i+1}^{*}\left(\theta_{i+1}^{*}\right)} & =\frac{\psi_{i}\left(\theta_{i}^{*}-\theta_{0}^{*}\right)}{\left(\theta_{i+1}^{*}-\theta_{i}^{*}\right)\left(\theta_{i+1}^{*}-\theta_{i-1}^{*}\right)}, \\
\frac{\eta_{d-i}\left(\theta_{i}\right)}{\eta_{d-i+1}\left(\theta_{i-1}\right)} & =\frac{\psi_{d-i}\left(\theta_{i}-\theta_{d}\right)}{\left(\theta_{i-1}-\theta_{i}\right)\left(\theta_{i-1}-\theta_{i+1}\right)}, \\
\frac{\eta_{d-i}^{*}\left(\theta_{i}^{*}\right)}{\eta_{d-i+1}^{*}\left(\theta_{i-1}^{*}\right)} & =\frac{\psi_{d-i}\left(\theta_{i}^{*}-\theta_{d}^{*}\right)}{\left(\theta_{i-1}^{*}-\theta_{i}^{*}\right)\left(\theta_{i-1}^{*}-\theta_{i+1}^{*}\right)} .
\end{aligned}
$$

Proof The result is vacuous for $d \leq 1$ and trivial for $d=2$. Next assume that $d \geq 3$. From the data in Lemma 19.5, we see that for $1 \leq i \leq d-1$ the scalar $\psi_{i}$ depends only on $i$ and $\beta$. Consequently $\psi_{i}$ is unchanged if we replace $\Phi$ by $\Phi^{*}$ or $\Phi^{\Downarrow}$. The result follows from these comments and (79).

Proposition 19.8 For $d \geq 1$ we have 


$$
\begin{aligned}
b_{0} & =\frac{\varphi_{1}}{\theta_{1}^{*}-\theta_{0}^{*}}, \\
b_{i} & =\frac{\varphi_{i+1} \psi_{i}\left(\theta_{i}^{*}-\theta_{0}^{*}\right)}{\left(\theta_{i+1}^{*}-\theta_{i}^{*}\right)\left(\theta_{i+1}^{*}-\theta_{i-1}^{*}\right)} \quad(1 \leq i \leq d-1), \\
c_{i} & =\frac{\phi_{i} \psi_{d-i}\left(\theta_{i}^{*}-\theta_{d}^{*}\right)}{\left(\theta_{i-1}^{*}-\theta_{i}^{*}\right)\left(\theta_{i-1}^{*}-\theta_{i+1}^{*}\right)} \quad(1 \leq i \leq d-1), \\
c_{d} & =\frac{\phi_{d}}{\theta_{d-1}^{*}-\theta_{d}^{*}}
\end{aligned}
$$

and

$$
\begin{aligned}
b_{0}^{*} & =\frac{\varphi_{1}}{\theta_{1}-\theta_{0}}, \\
b_{i}^{*} & =\frac{\varphi_{i+1} \psi_{i}\left(\theta_{i}-\theta_{0}\right)}{\left(\theta_{i+1}-\theta_{i}\right)\left(\theta_{i+1}-\theta_{i-1}\right)} \quad(1 \leq i \leq d-1), \\
c_{i}^{*} & =\frac{\phi_{d-i+1} \psi_{d-i}\left(\theta_{i}-\theta_{d}\right)}{\left(\theta_{i-1}-\theta_{i}\right)\left(\theta_{i-1}-\theta_{i+1}\right)} \quad(1 \leq i \leq d-1), \\
c_{d}^{*} & =\frac{\phi_{1}}{\theta_{d-1}-\theta_{d}} .
\end{aligned}
$$

Proof Evaluate the formulas in Lemma 19.4 using (79) and Corollary 19.7.

We mention some attractive formulas involving the intersection numbers; similar formulas apply to the dual intersection numbers.

Lemma 19.9 The following hold:

(i) for $0 \leq i \leq d-1$,

$$
\frac{\theta_{0}+\theta_{1}+\cdots+\theta_{i}-a_{0}-a_{1}-\cdots-a_{i}}{b_{i}}=\prod_{h=0}^{i-1} \frac{\theta_{i+1}^{*}-\theta_{h}^{*}}{\theta_{i}^{*}-\theta_{h}^{*}} ;
$$

(ii) for $1 \leq i \leq d$,

$$
\frac{a_{0}+a_{1}+\cdots+a_{i-1}-\theta_{d}-\theta_{d-1}-\cdots-\theta_{d-i+1}}{c_{i}}=\prod_{h=i+1}^{d} \frac{\theta_{i-1}^{*}-\theta_{h}^{*}}{\theta_{i}^{*}-\theta_{h}^{*}} .
$$

\section{Proof}

(i) To verify (80), eliminate $b_{i}$ using Lemma 19.4(i), and evaluate the result using Lemma 8.2.

(ii) To verify (81), eliminate $c_{i}$ using Lemma 19.4(ii), and evaluate the result using Lemma 9.5 . 
Corollary 19.10 For $d \geq 1$,

$$
\begin{gathered}
b_{d-1}=\left(a_{d}-\theta_{d}\right) \frac{\left(\theta_{d-1}^{*}-\theta_{0}^{*}\right)\left(\theta_{d-1}^{*}-\theta_{1}^{*}\right) \cdots\left(\theta_{d-1}^{*}-\theta_{d-2}^{*}\right)}{\left(\theta_{d}^{*}-\theta_{0}^{*}\right)\left(\theta_{d}^{*}-\theta_{1}^{*}\right) \cdots\left(\theta_{d}^{*}-\theta_{d-2}^{*}\right)}, \\
c_{1}=\left(a_{0}-\theta_{d}\right) \frac{\left(\theta_{1}^{*}-\theta_{2}^{*}\right)\left(\theta_{1}^{*}-\theta_{3}^{*}\right) \cdots\left(\theta_{1}^{*}-\theta_{d}^{*}\right)}{\left(\theta_{0}^{*}-\theta_{2}^{*}\right)\left(\theta_{0}^{*}-\theta_{3}^{*}\right) \cdots\left(\theta_{0}^{*}-\theta_{d}^{*}\right)} .
\end{gathered}
$$

Proof To get (82) set $i=d-1$ in (80), and simplify the result using (4). To get (83) set $i=1$ in (81).

Next we obtain $\left\{\theta_{i}\right\}_{i=0}^{d}$ in terms of the intersection numbers and dual eigenvalues. We give two versions, that resemble [3, Corollary 8.3.3].

Lemma 19.11 For $d \geq 1$,

$$
\begin{aligned}
\theta_{0} & =a_{0}+b_{0}, \\
\theta_{i} & =a_{i}+b_{i} \prod_{h=0}^{i-1} \frac{\theta_{i+1}^{*}-\theta_{h}^{*}}{\theta_{i}^{*}-\theta_{h}^{*}}-b_{i-1} \prod_{h=0}^{i-2} \frac{\theta_{i}^{*}-\theta_{h}^{*}}{\theta_{i-1}^{*}-\theta_{h}^{*}} \quad(1 \leq i \leq d-1), \\
\theta_{d} & =a_{d}-b_{d-1} \prod_{h=0}^{d-2} \frac{\theta_{d}^{*}-\theta_{h}^{*}}{\theta_{d-1}^{*}-\theta_{h}^{*}} .
\end{aligned}
$$

Proof To find $\theta_{i}$ for $0 \leq i \leq d-1$, solve (80) for $\theta_{i}$ and use induction on $i$. The formula for $\theta_{d}$ comes from (82).

Lemma 19.12 For $d \geq 1$,

$$
\begin{aligned}
\theta_{0} & =a_{d}+c_{d}, \\
\theta_{i} & =a_{d-i}+c_{d-i} \prod_{h=d-i+1}^{d} \frac{\theta_{d-i-1}^{*}-\theta_{h}^{*}}{\theta_{d-i}^{*}-\theta_{h}^{*}}-c_{d-i+1} \prod_{h=d-i+2}^{d} \frac{\theta_{d-i}^{*}-\theta_{h}^{*}}{\theta_{d-i+1}^{*}-\theta_{h}^{*}} \quad(1 \leq i \leq d-1), \\
\theta_{d} & =a_{0}-c_{1} \prod_{h=2}^{d} \frac{\theta_{0}^{*}-\theta_{h}^{*}}{\theta_{1}^{*}-\theta_{h}^{*}} .
\end{aligned}
$$

Proof To find $\theta_{d}, \theta_{d-1}, \ldots, \theta_{1}$ use (81). The formula for $\theta_{0}$ comes from (69) at $i=d$.

Next we obtain some results about duality.

Lemma 19.13 For $0 \leq i, j, r, s \leq d$ such that $i+j=r+s$ and $r \neq s$, 


$$
\frac{\theta_{i}-\theta_{j}}{\theta_{r}-\theta_{s}}=\frac{\theta_{i}^{*}-\theta_{j}^{*}}{\theta_{r}^{*}-\theta_{s}^{*}}
$$

Proof Use the data in Lemma 12.3.

Lemma 19.14 For $0 \leq i \leq d-1$,

$$
\begin{aligned}
b_{i} & \frac{\left(\theta_{i+1}^{*}-\theta_{0}^{*}\right)\left(\theta_{i+1}^{*}-\theta_{1}^{*}\right) \cdots\left(\theta_{i+1}^{*}-\theta_{i}^{*}\right)}{\left(\theta_{i}^{*}-\theta_{0}^{*}\right)\left(\theta_{i}^{*}-\theta_{1}^{*}\right) \cdots\left(\theta_{i}^{*}-\theta_{i-1}^{*}\right)} \\
& =b_{i}^{*} \frac{\left(\theta_{i+1}-\theta_{0}\right)\left(\theta_{i+1}-\theta_{1}\right) \cdots\left(\theta_{i+1}-\theta_{i}\right)}{\left(\theta_{i}-\theta_{0}\right)\left(\theta_{i}-\theta_{1}\right) \cdots\left(\theta_{i}-\theta_{i-1}\right)} .
\end{aligned}
$$

Proof Each side is equal to $\varphi_{i+1}$ by Lemma 19.4(i),(iii).

Lemma 19.15 For $d \geq 1$,

$$
\begin{aligned}
b_{0}\left(\theta_{1}^{*}-\theta_{0}^{*}\right) & =b_{0}^{*}\left(\theta_{1}-\theta_{0}\right), \\
\frac{b_{i}\left(\theta_{i+1}^{*}-\theta_{i}^{*}\right)\left(\theta_{i+1}^{*}-\theta_{i-1}^{*}\right)}{\theta_{i}^{*}-\theta_{0}^{*}} & =\frac{b_{i}^{*}\left(\theta_{i+1}-\theta_{i}\right)\left(\theta_{i+1}-\theta_{i-1}\right)}{\theta_{i}-\theta_{0}} \quad(1 \leq i \leq d-1) .
\end{aligned}
$$

Proof Compare the formulas for $b_{i}, b_{i}^{*}$ in Proposition 19.8.

Lemma 19.16 For $0 \leq i \leq d-1$,

$$
\begin{aligned}
c_{i+1} & \frac{\left(\theta_{i}^{*}-\theta_{d}^{*}\right)\left(\theta_{i}^{*}-\theta_{d-1}^{*}\right) \cdots\left(\theta_{i}^{*}-\theta_{i+1}^{*}\right)}{\left(\theta_{i+1}^{*}-\theta_{d}^{*}\right)\left(\theta_{i+1}^{*}-\theta_{d-1}^{*}\right) \cdots\left(\theta_{i+1}^{*}-\theta_{i+2}^{*}\right)} \\
= & c_{d-i}^{*} \frac{\left(\theta_{d-i-1}-\theta_{d}\right)\left(\theta_{d-i-1}-\theta_{d-1}\right) \cdots\left(\theta_{d-i-1}-\theta_{d-i}\right)}{\left(\theta_{d-i}-\theta_{d}\right)\left(\theta_{d-i}-\theta_{d-1}\right) \cdots\left(\theta_{d-i}-\theta_{d-i+1}\right)} .
\end{aligned}
$$

Proof Each side is equal to $\phi_{i+1}$ by Lemma 19.4(ii),(iv).

Lemma 19.17 For $0 \leq i \leq d-1$, 


$$
\begin{gathered}
\sum_{h=0}^{i} \frac{\theta_{h}-a_{h}}{\theta_{i}-\theta_{i+1}}=\sum_{h=0}^{i} \frac{\theta_{h}^{*}-a_{h}^{*}}{\theta_{i}^{*}-\theta_{i+1}^{*}}, \\
\sum_{h=0}^{i} \frac{\theta_{d-h}-a_{h}}{\theta_{d-i}-\theta_{d-i-1}}=\sum_{h=0}^{i} \frac{\theta_{h}^{*}-a_{d-h}^{*}}{\theta_{i}^{*}-\theta_{i+1}^{*}}, \\
\sum_{h=0}^{i} \frac{\theta_{h}-a_{d-h}}{\theta_{i}-\theta_{i+1}}=\sum_{h=0}^{i} \frac{\theta_{d-h}^{*}-a_{h}^{*}}{\theta_{d-i}^{*}-\theta_{d-i-1}^{*}}, \\
\sum_{h=0}^{i} \frac{\theta_{d-h}-a_{d-h}}{\theta_{d-i}-\theta_{d-i-1}}=\sum_{h=0}^{i} \frac{\theta_{d-h}^{*}-a_{d-h}^{*}}{\theta_{d-i}^{*}-\theta_{d-i-1}^{*}} .
\end{gathered}
$$

Proof The first equation holds, because each side is equal to $-\varphi_{i+1}\left(\theta_{i+1}-\right.$ $\left.\theta_{i}\right)^{-1}\left(\theta_{i+1}^{*}-\theta_{i}^{*}\right)^{-1}$ by Lemma 8.2. The remaining equations are similarly obtained.

We emphasize a special case of Lemma 19.17.

Lemma 19.18 For $d \geq 1$,

$$
\begin{array}{lrl}
\frac{\theta_{0}-a_{0}}{\theta_{0}-\theta_{1}}=\frac{\theta_{0}^{*}-a_{0}^{*}}{\theta_{0}^{*}-\theta_{1}^{*}}, & \frac{\theta_{d}-a_{0}}{\theta_{d}-\theta_{d-1}}=\frac{\theta_{0}^{*}-a_{d}^{*}}{\theta_{0}^{*}-\theta_{1}^{*}} \\
\frac{\theta_{0}-a_{d}}{\theta_{0}-\theta_{1}}=\frac{\theta_{d}^{*}-a_{0}^{*}}{\theta_{d}^{*}-\theta_{d-1}^{*}}, & \frac{\theta_{d}-a_{d}}{\theta_{d}-\theta_{d-1}}=\frac{\theta_{d}^{*}-a_{d}^{*}}{\theta_{d}^{*}-\theta_{d-1}^{*}} .
\end{array}
$$

Proof Set $i=0$ in Lemma 19.17 .

Motivated by Lemma 10.3 , our next goal is to explicitly write the intersection numbers and dual intersection numbers in terms of $\varphi_{1}$ and $\left\{\theta_{i}\right\}_{i=0}^{d},\left\{\theta_{i}^{*}\right\}_{i=0}^{d}$. To avoid trivialities we assume that $d \geq 2$. We will use the following result, which appears in [4, Lemma 15.16] in the context of distance-regular graphs.

Lemma 19.19 For $d \geq 2, \varphi_{2}$ is equal to each of

$$
\begin{aligned}
& \varphi_{1} \frac{\theta_{0}+\theta_{1}-\theta_{d-1}-\theta_{d}}{\theta_{0}-\theta_{d}}+\left(\theta_{0}^{*}-\theta_{1}^{*}\right)\left(\theta_{0}+\theta_{1}-\theta_{d-1}-\theta_{d}\right)+\left(\theta_{2}^{*}-\theta_{0}^{*}\right)\left(\theta_{1}-\theta_{d}\right), \\
& \varphi_{1} \frac{\theta_{0}^{*}+\theta_{1}^{*}-\theta_{d-1}^{*}-\theta_{d}^{*}}{\theta_{0}^{*}-\theta_{d}^{*}}+\left(\theta_{0}-\theta_{1}\right)\left(\theta_{0}^{*}+\theta_{1}^{*}-\theta_{d-1}^{*}-\theta_{d}^{*}\right)+\left(\theta_{2}-\theta_{0}\right)\left(\theta_{1}^{*}-\theta_{d}^{*}\right) .
\end{aligned}
$$


Proof To get (84), evaluate (PA3) at $i=2$ and simplify the result using (PA4) at $i=1$. To get (85) from (84), note that each of $\varphi_{1}, \varphi_{2}$ is unchanged if we replace $\Phi$ by $\Phi^{*}$.

The following result appears in [3, Theorem 8.1.1] and [4, Theorem 15.18], in the context of distance-regular graphs.

\section{Proposition 19.20 For $d \geq 2$,}

$$
\begin{aligned}
b_{0} & =\frac{\varphi_{1}}{\theta_{1}^{*}-\theta_{0}^{*},} \\
b_{i} & =\frac{\left(\theta_{0}^{*}-\theta_{i}^{*}\right)\left(\varphi_{1} f_{i}^{-}+g_{i}^{-}\right)}{\left(\theta_{i+1}^{*}-\theta_{i}^{*}\right)\left(\theta_{i+1}^{*}-\theta_{i-1}^{*}\right)} \quad(1 \leq i \leq d-1), \\
c_{i} & =\frac{\left(\theta_{0}^{*}-\theta_{i}^{*}\right)\left(\varphi_{1} f_{i}^{+}+g_{i}^{+}\right)}{\left(\theta_{i-1}^{*}-\theta_{i}^{*}\right)\left(\theta_{i-1}^{*}-\theta_{i+1}^{*}\right)} \quad(1 \leq i \leq d-1), \\
c_{d} & =\frac{\varphi_{1}+\left(\theta_{0}-\theta_{1}\right)\left(\theta_{0}^{*}-\theta_{d}^{*}\right)}{\theta_{d-1}^{*}-\theta_{d}^{*}}
\end{aligned}
$$

where

$$
\begin{aligned}
& f_{i}^{ \pm}=\frac{\theta_{1}^{*}-\theta_{i \pm 1}^{*}}{\theta_{0}^{*}-\theta_{i}^{*}}-\frac{\theta_{1}^{*}-\theta_{d-1}^{*}}{\theta_{0}^{*}-\theta_{d}^{*}}, \\
& g_{i}^{ \pm}=\left(\theta_{1}-\theta_{2}\right)\left(\theta_{i}^{*}-\theta_{d}^{*}\right)-\left(\theta_{0}-\theta_{1}\right)\left(\theta_{i \pm 1}^{*}-\theta_{d-1}^{*}\right) .
\end{aligned}
$$

To get $\left\{b_{i}^{*}\right\}_{i=0}^{d-1}$ and $\left\{c_{i}^{*}\right\}_{i=1}^{d}$, exchange the symbols $\theta, \theta^{*}$ everywhere in the above equations.

Proof To get $b_{0}$, set $i=0$ in Lemma 19.4(i). To get $c_{d}$, set $i=d$ in Lemma 19.4(ii) and eliminate $\phi_{d}$ using (PA4). We now compute $b_{i}, c_{i}$ for $1 \leq i \leq d-1$. Let $i$ be given. For the equations (74) at $j=1$ and $j=2$, eliminate $a_{i}$ using (69) to obtain a system of two linear equations in the unknowns $b_{i}, c_{i}$. Using linear algebra we routinely solve this system for $b_{i}, c_{i}$, and in the solution eliminate $\varphi_{2}$ using (85). We have obtained $\left\{b_{i}\right\}_{i=0}^{d-1}$ and $\left\{c_{i}\right\}_{i=1}^{d}$. To obtain $\left\{b_{i}^{*}\right\}_{i=0}^{d-1}$ and $\left\{c_{i}^{*}\right\}_{i=1}^{d}$, apply the above arguments to $\Phi^{*}$.

Our next goal is to give a variation on Proposition 19.20 that involves $a_{0}, a_{0}^{*}$ and $c_{1}, c_{1}^{*}$.

Lemma 19.21 For $d \geq 2, \varphi_{2}$ is equal to each of

$$
\left(c_{1}-a_{0}+\theta_{1}\right)\left(\theta_{2}^{*}-\theta_{0}^{*}\right), \quad\left(c_{1}^{*}-a_{0}^{*}+\theta_{1}^{*}\right)\left(\theta_{2}-\theta_{0}\right) .
$$

Proof Set $i=1$ in (69), Lemma 19.4(i), (80). The resulting equations show that $\varphi_{2}$ is equal to the expression on the left in (86). Note that $\varphi_{2}$ is unchanged if we replace $\Phi$ by $\Phi^{*}$. Therefore $\varphi_{2}$ is equal to the expression on the right in (86).

We clarify how $c_{1}, a_{0}$ are related and how $c_{1}^{*}, a_{0}^{*}$ are related. 
Lemma 19.22 For $d \geq 2$ we have

$$
\begin{aligned}
& c_{1}=\left(\theta_{d}-a_{0}\right) \frac{\left(\theta_{0}^{*}-\theta_{1}^{*}\right)\left(\theta_{1}-\theta_{d-1}\right)-\left(\theta_{1}^{*}-\theta_{2}^{*}\right)\left(\theta_{0}-\theta_{d}\right)}{\left(\theta_{0}^{*}-\theta_{2}^{*}\right)\left(\theta_{0}-\theta_{d}\right)}, \\
& c_{1}^{*}=\left(\theta_{d}^{*}-a_{0}^{*}\right) \frac{\left(\theta_{0}-\theta_{1}\right)\left(\theta_{1}^{*}-\theta_{d-1}^{*}\right)-\left(\theta_{1}-\theta_{2}\right)\left(\theta_{0}^{*}-\theta_{d}^{*}\right)}{\left(\theta_{0}-\theta_{2}\right)\left(\theta_{0}^{*}-\theta_{d}^{*}\right)} .
\end{aligned}
$$

Proof To verify the first equation, express the left-hand side in terms of $\varphi_{2}$ using Lemma 19.21, and in the resulting equation express $a_{0}$ in terms of $\varphi_{1}$ using Lemma 8.1. Evaluate the result using (84). We have verified the first equation. To get the second equation, apply the first equation to $\Phi^{*}$.

Proposition 19.23 For $d \geq 2$,

$$
\begin{aligned}
b_{0} & =\frac{\left(\theta_{0}^{*}-a_{0}^{*}\right)\left(\theta_{1}-\theta_{0}\right)}{\theta_{1}^{*}-\theta_{0}^{*}}, \\
b_{i} & =\frac{c_{1}^{*}\left(\theta_{0}^{*}-\theta_{i}^{*}\right)\left(\theta_{0}-\theta_{2}\right)+\left(\theta_{i}^{*}-a_{0}^{*}\right)\left(\left(\theta_{1}-\theta_{2}\right)\left(\theta_{0}^{*}-\theta_{i}^{*}\right)-\left(\theta_{0}-\theta_{1}\right)\left(\theta_{1}^{*}-\theta_{i-1}^{*}\right)\right)}{\left(\theta_{i+1}^{*}-\theta_{i}^{*}\right)\left(\theta_{i+1}^{*}-\theta_{i-1}^{*}\right)}, \\
c_{i} & =\frac{c_{1}^{*}\left(\theta_{0}^{*}-\theta_{i}^{*}\right)\left(\theta_{0}-\theta_{2}\right)+\left(\theta_{i}^{*}-a_{0}^{*}\right)\left(\left(\theta_{1}-\theta_{2}\right)\left(\theta_{0}^{*}-\theta_{i}^{*}\right)-\left(\theta_{0}-\theta_{1}\right)\left(\theta_{1}^{*}-\theta_{i+1}^{*}\right)\right)}{\left(\theta_{i-1}^{*}-\theta_{i}^{*}\right)\left(\theta_{i-1}^{*}-\theta_{i+1}^{*}\right)}, \\
c_{d} & =\frac{\left(\theta_{d}^{*}-a_{0}^{*}\right)\left(\theta_{1}-\theta_{0}\right)}{\theta_{d-1}^{*}-\theta_{d}^{*}} .
\end{aligned}
$$

To get $\left\{b_{i}^{*}\right\}_{i=0}^{d-1}$ and $\left\{c_{i}^{*}\right\}_{i=1}^{d}$, exchange starred and nonstarred symbols everywhere in the above equations.

Proof To get $b_{0}$, set $i=0$ in Lemma 19.4(i) and evaluate the result using $\varphi_{1}=\left(\theta_{0}^{*}-a_{0}^{*}\right)\left(\theta_{1}-\theta_{0}\right)$. To get $c_{d}$, set $i=d$ in Lemma 19.4(ii) and evaluate the result using $\phi_{d}=\left(\theta_{d}^{*}-a_{0}^{*}\right)\left(\theta_{1}-\theta_{0}\right)$. To obtain $b_{i}, c_{i}$ for $1 \leq i \leq d-1$, we proceed as in the proof of Proposition 19.20. Let $i$ be given. For the equations (74) at $j=1$ and $j=2$, eliminate $a_{i}$ using (69) to obtain a system of two linear equations in the unknowns $b_{i}, c_{i}$. Using linear algebra we routinely solve this system for $b_{i}, c_{i}$, and in the solution eliminate $\varphi_{1}, \quad \varphi_{2}$ using $\varphi_{1}=\left(\theta_{0}^{*}-a_{0}^{*}\right)\left(\theta_{1}-\theta_{0}\right) \quad$ and $\varphi_{2}=\left(c_{1}^{*}-a_{0}^{*}+\theta_{1}^{*}\right)\left(\theta_{2}-\theta_{0}\right)$. We have obtained $\left\{b_{i}\right\}_{i=0}^{d-1}$ and $\left\{c_{i}\right\}_{i=1}^{d}$. To obtain $\left\{b_{i}^{*}\right\}_{i=0}^{d-1}$ and $\left\{c_{i}^{*}\right\}_{i=1}^{d}$, apply the above arguments to $\Phi^{*}$.

Note 19.24 Lemma 19.22 and Proposition 19.23 effectively give the intersection numbers (resp. dual intersection numbers) in terms of $a_{0}^{*}$ (resp. $a_{0}$ ) and $\left\{\theta_{i}\right\}_{i=0}^{d}$, $\left\{\theta_{i}^{*}\right\}_{i=0}^{d}$. The scalars $a_{0}$ and $a_{0}^{*}$ are related by the first equation in Lemma 19.18.

Note 19.25 There is a Leonard system attached to the Bose-Mesner algebra of a $Q$ polynomial distance-regular graph; see [2, p. 260], [12]. For this Leonard system $a_{0}=0, a_{0}^{*}=0$ and $c_{1}=1, c_{1}^{*}=1$. 
Open Access This article is licensed under a Creative Commons Attribution 4.0 International License, which permits use, sharing, adaptation, distribution and reproduction in any medium or format, as long as you give appropriate credit to the original author(s) and the source, provide a link to the Creative Commons licence, and indicate if changes were made. The images or other third party material in this article are included in the article's Creative Commons licence, unless indicated otherwise in a credit line to the material. If material is not included in the article's Creative Commons licence and your intended use is not permitted by statutory regulation or exceeds the permitted use, you will need to obtain permission directly from the copyright holder. To view a copy of this licence, visit http:// creativecommons.org/licenses/by/4.0/.

\section{Appendix: Parameter arrays and Intersection Numbers}

The parameter arrays are listed in [21, Sect. 5]. In this appendix we go through the list, and for each parameter array we give the corresponding intersection numbers and dual intersection numbers.

Example 20.1 ( $q$-Racah). We have

$$
\begin{aligned}
& \theta_{i}=\theta_{0}+h\left(1-q^{i}\right)\left(1-s q^{i+1}\right) q^{-i} \\
& \theta_{i}^{*}=\theta_{0}^{*}+h^{*}\left(1-q^{i}\right)\left(1-s^{*} q^{i+1}\right) q^{-i}
\end{aligned}
$$

for $0 \leq i \leq d$ and

$$
\begin{aligned}
& \varphi_{i}=h h^{*} q^{1-2 i}\left(1-q^{i}\right)\left(1-q^{i-d-1}\right)\left(1-r_{1} q^{i}\right)\left(1-r_{2} q^{i}\right), \\
& \phi_{i}=h h^{*} q^{1-2 i}\left(1-q^{i}\right)\left(1-q^{i-d-1}\right)\left(r_{1}-s^{*} q^{i}\right)\left(r_{2}-s^{*} q^{i}\right) / s^{*}
\end{aligned}
$$

for $1 \leq i \leq d$, with $r_{1} r_{2}=s s^{*} q^{d+1}$. We have

$$
\begin{aligned}
& b_{0}=\frac{h\left(1-q^{-d}\right)\left(1-r_{1} q\right)\left(1-r_{2} q\right)}{1-s^{*} q^{2}} \text {, } \\
& b_{i}=\frac{h\left(1-q^{i-d}\right)\left(1-s^{*} q^{i+1}\right)\left(1-r_{1} q^{i+1}\right)\left(1-r_{2} q^{i+1}\right)}{\left(1-s^{*} q^{2 i+1}\right)\left(1-s^{*} q^{2 i+2}\right)} \quad(1 \leq i \leq d-1), \\
& c_{i}=\frac{h\left(1-q^{i}\right)\left(1-s^{*} q^{i+d+1}\right)\left(r_{1}-s^{*} q^{i}\right)\left(r_{2}-s^{*} q^{i}\right)}{s^{*} q^{d}\left(1-s^{*} q^{2 i}\right)\left(1-s^{*} q^{2 i+1}\right)} \quad(1 \leq i \leq d-1), \\
& c_{d}=\frac{h\left(1-q^{d}\right)\left(r_{1}-s^{*} q^{d}\right)\left(r_{2}-s^{*} q^{d}\right)}{s^{*} q^{d}\left(1-s^{*} q^{2 d}\right)} .
\end{aligned}
$$

To get $\left\{b_{i}^{*}\right\}_{i=0}^{d-1}$ and $\left\{c_{i}^{*}\right\}_{i=1}^{d}$, in the above formulas exchange $h \leftrightarrow h^{*}, s \leftrightarrow s^{*}$ and preserve $r_{1}, r_{2}, q$.

Example 20.2 ( $q$-Hahn). We have 


$$
\begin{aligned}
\theta_{i} & =\theta_{0}+h\left(1-q^{i}\right) q^{-i} \\
\theta_{i}^{*} & =\theta_{0}^{*}+h^{*}\left(1-q^{i}\right)\left(1-s^{*} q^{i+1}\right) q^{-i}
\end{aligned}
$$

for $0 \leq i \leq d$ and

$$
\begin{aligned}
& \varphi_{i}=h h^{*} q^{1-2 i}\left(1-q^{i}\right)\left(1-q^{i-d-1}\right)\left(1-r q^{i}\right), \\
& \phi_{i}=-h h^{*} q^{1-i}\left(1-q^{i}\right)\left(1-q^{i-d-1}\right)\left(r-s^{*} q^{i}\right)
\end{aligned}
$$

for $1 \leq i \leq d$. We have

$$
\begin{aligned}
b_{0} & =\frac{h\left(1-q^{-d}\right)(1-r q)}{1-s^{*} q^{2}}, \\
b_{i} & =\frac{h\left(1-q^{i-d}\right)\left(1-s^{*} q^{i+1}\right)\left(1-r q^{i+1}\right)}{\left(1-s^{*} q^{2 i+1}\right)\left(1-s^{*} q^{2 i+2}\right)} \quad(0 \leq i \leq d-1), \\
c_{i} & =\frac{-h q^{i-d}\left(1-q^{i}\right)\left(1-s^{*} q^{i+d+1}\right)\left(r-s^{*} q^{i}\right)}{\left(1-s^{*} q^{2 i}\right)\left(1-s^{*} q^{2 i+1}\right)} \quad(1 \leq i \leq d), \\
c_{d} & =\frac{-h\left(1-q^{d}\right)\left(r-s^{*} q^{d}\right)}{1-s^{*} q^{2 d}}
\end{aligned}
$$

and

$$
\begin{aligned}
& b_{i}^{*}=h^{*}\left(1-q^{i-d}\right)\left(1-r q^{i+1}\right) \quad(0 \leq i \leq d-1), \\
& c_{i}^{*}=h^{*}\left(1-q^{i}\right)\left(q s^{*}-r q^{i-d}\right) \quad(1 \leq i \leq d) .
\end{aligned}
$$

Example 20.3 (Dual $q$-Hahn). We have

$$
\begin{aligned}
& \theta_{i}=\theta_{0}+h\left(1-q^{i}\right)\left(1-s q^{i+1}\right) q^{-i} \\
& \theta_{i}^{*}=\theta_{0}^{*}+h^{*}\left(1-q^{i}\right) q^{-i}
\end{aligned}
$$

for $0 \leq i \leq d$ and

$$
\begin{aligned}
& \varphi_{i}=h h^{*} q^{1-2 i}\left(1-q^{i}\right)\left(1-q^{i-d-1}\right)\left(1-r q^{i}\right), \\
& \phi_{i}=h h^{*} q^{d+2-2 i}\left(1-q^{i}\right)\left(1-q^{i-d-1}\right)\left(s-r q^{i-d-1}\right)
\end{aligned}
$$

for $1 \leq i \leq d$. We have

$$
\begin{aligned}
b_{i}=h\left(1-q^{i-d}\right)\left(1-r q^{i+1}\right) & (0 \leq i \leq d-1), \\
c_{i}=h\left(1-q^{i}\right)\left(q s-r q^{i-d}\right) & (1 \leq i \leq d)
\end{aligned}
$$

and 


$$
\begin{aligned}
& b_{0}^{*}=\frac{h^{*}\left(1-q^{-d}\right)(1-r q)}{1-s q^{2}}, \\
& b_{i}^{*}=\frac{h^{*}\left(1-q^{i-d}\right)\left(1-s q^{i+1}\right)\left(1-r q^{i+1}\right)}{\left(1-s q^{2 i+1}\right)\left(1-s q^{2 i+2}\right)} \quad(1 \leq i \leq d-1), \\
& c_{i}^{*}=\frac{-h^{*} q^{i-d}\left(1-q^{i}\right)\left(1-s q^{i+d+1}\right)\left(r-s q^{i}\right)}{\left(1-s q^{2 i}\right)\left(1-s q^{2 i+1}\right)} \quad(1 \leq i \leq d-1), \\
& c_{d}^{*}=\frac{-h^{*}\left(1-q^{d}\right)\left(r-s q^{d}\right)}{1-s q^{2 d}} .
\end{aligned}
$$

Example 20.4 (quantum $q$-Krawtchouk). We have

$$
\begin{aligned}
\theta_{i} & =\theta_{0}-s q\left(1-q^{i}\right), \\
\theta_{i}^{*} & =\theta_{0}^{*}+h^{*}\left(1-q^{i}\right) q^{-i}
\end{aligned}
$$

for $0 \leq i \leq d$ and

$$
\begin{aligned}
& \varphi_{i}=-r h^{*} q^{1-i}\left(1-q^{i}\right)\left(1-q^{i-d-1}\right), \\
& \phi_{i}=h^{*} q^{d+2-2 i}\left(1-q^{i}\right)\left(1-q^{i-d-1}\right)\left(s-r q^{i-d-1}\right)
\end{aligned}
$$

for $1 \leq i \leq d$. We have

$$
\begin{array}{rlr}
b_{i}=-r q^{i+1}\left(1-q^{i-d}\right) & (0 \leq i \leq d-1), \\
c_{i}=\left(1-q^{i}\right)\left(q s-r q^{i-d}\right) & (1 \leq i \leq d)
\end{array}
$$

and

$$
\begin{array}{rlr}
b_{i}^{*} & =\frac{h^{*} r\left(1-q^{i-d}\right)}{s q^{2 i+1}} & (0 \leq i \leq d-1), \\
c_{i}^{*} & =\frac{h^{*}\left(1-q^{i}\right)\left(r-s q^{i}\right)}{s q^{2 i}} & (1 \leq i \leq d) .
\end{array}
$$

Example 20.5 ( $q$-Krawtchouk). We have

$$
\begin{aligned}
& \theta_{i}=\theta_{0}+h\left(1-q^{i}\right) q^{-i} \\
& \theta_{i}^{*}=\theta_{0}^{*}+h^{*}\left(1-q^{i}\right)\left(1-s^{*} q^{i+1}\right) q^{-i}
\end{aligned}
$$

for $0 \leq i \leq d$ and 


$$
\begin{aligned}
& \varphi_{i}=h h^{*} q^{1-2 i}\left(1-q^{i}\right)\left(1-q^{i-d-1}\right), \\
& \phi_{i}=h h^{*} s^{*} q\left(1-q^{i}\right)\left(1-q^{i-d-1}\right)
\end{aligned}
$$

for $1 \leq i \leq d$. We have

$$
\begin{aligned}
b_{0} & =\frac{h\left(1-q^{-d}\right)}{1-s^{*} q^{2}}, \\
b_{i} & =\frac{h\left(1-q^{i-d}\right)\left(1-s^{*} q^{i+1}\right)}{\left(1-s^{*} q^{2 i+1}\right)\left(1-s^{*} q^{2 i+2}\right)} \quad(1 \leq i \leq d-1), \\
c_{i} & =\frac{h s^{*} q^{2 i-d}\left(1-q^{i}\right)\left(1-s^{*} q^{i+d+1}\right)}{\left(1-s^{*} q^{2 i}\right)\left(1-s^{*} q^{2 i+1}\right)} \quad(1 \leq i \leq d-1), \\
c_{d} & =\frac{h s^{*} q^{d}\left(1-q^{d}\right)}{1-s^{*} q^{2 d}}
\end{aligned}
$$

and

$$
\begin{aligned}
b_{i}^{*}=h^{*}\left(1-q^{i-d}\right) & (0 \leq i \leq d-1), \\
c_{i}^{*}=h^{*} s^{*} q\left(1-q^{i}\right) & (1 \leq i \leq d) .
\end{aligned}
$$

Example 20.6 (affine $q$-Krawtchouk). We have

$$
\begin{aligned}
\theta_{i} & =\theta_{0}+h\left(1-q^{i}\right) q^{-i}, \\
\theta_{i}^{*} & =\theta_{0}^{*}+h^{*}\left(1-q^{i}\right) q^{-i}
\end{aligned}
$$

for $0 \leq i \leq d$ and

$$
\begin{aligned}
& \varphi_{i}=h h^{*} q^{1-2 i}\left(1-q^{i}\right)\left(1-q^{i-d-1}\right)\left(1-r q^{i}\right), \\
& \phi_{i}=-h h^{*} r q^{1-i}\left(1-q^{i}\right)\left(1-q^{i-d-1}\right)
\end{aligned}
$$

for $1 \leq i \leq d$. We have

$$
\begin{array}{rlr}
b_{i}=h\left(1-q^{i-d}\right)\left(1-r q^{i+1}\right) & (0 \leq i \leq d-1), \\
c_{i}=-h r q^{i-d}\left(1-q^{i}\right) & (1 \leq i \leq d) .
\end{array}
$$

To get $\left\{b_{i}^{*}\right\}_{i=0}^{d-1}$ and $\left\{c_{i}^{*}\right\}_{i=1}^{d}$, in the above formulas exchange $h \leftrightarrow h^{*}$ and preserve $r$, $q$.

Example 20.7 (dual $q$-Krawtchouk). We have 


$$
\begin{aligned}
& \theta_{i}=\theta_{0}+h\left(1-q^{i}\right)\left(1-s q^{i+1}\right) q^{-i} \\
& \theta_{i}^{*}=\theta_{0}^{*}+h^{*}\left(1-q^{i}\right) q^{-i}
\end{aligned}
$$

for $0 \leq i \leq d$ and

$$
\begin{aligned}
& \varphi_{i}=h h^{*} q^{1-2 i}\left(1-q^{i}\right)\left(1-q^{i-d-1}\right) \\
& \phi_{i}=h h^{*} s q^{d+2-2 i}\left(1-q^{i}\right)\left(1-q^{i-d-1}\right)
\end{aligned}
$$

for $1 \leq i \leq d$. We have

$$
\begin{aligned}
& b_{i}=h\left(1-q^{i-d}\right) \quad(0 \leq i \leq d-1), \\
& c_{i}=h s q\left(1-q^{i}\right) \quad(1 \leq i \leq d)
\end{aligned}
$$

and

$$
\begin{aligned}
b_{0}^{*} & =\frac{h^{*}\left(1-q^{-d}\right)}{1-s q^{2}}, \\
b_{i}^{*} & =\frac{h^{*}\left(1-q^{i-d}\right)\left(1-s q^{i+1}\right)}{\left(1-s q^{2 i+1}\right)\left(1-s q^{2 i+2}\right)} \quad(1 \leq i \leq d-1), \\
c_{i}^{*} & =\frac{h^{*} s q^{2 i-d}\left(1-q^{i}\right)\left(1-s q^{i+d+1}\right)}{\left(1-s q^{2 i}\right)\left(1-s q^{2 i+1}\right)} \quad(1 \leq i \leq d-1), \\
c_{d}^{*} & =\frac{h^{*} s q^{d}\left(1-q^{d}\right)}{1-s q^{2 d}} .
\end{aligned}
$$

Example 20.8 (Racah). We have

$$
\begin{gathered}
\theta_{i}=\theta_{0}+h i(i+1+s), \\
\theta_{i}^{*}=\theta_{0}^{*}+h^{*} i\left(i+1+s^{*}\right)
\end{gathered}
$$

for $0 \leq i \leq d$ and

$$
\begin{aligned}
\varphi_{i} & =h h^{*} i(i-d-1)\left(i+r_{1}\right)\left(i+r_{2}\right), \\
\phi_{i} & =h h^{*} i(i-d-1)\left(i+s^{*}-r_{1}\right)\left(i+s^{*}-r_{2}\right)
\end{aligned}
$$

for $1 \leq i \leq d$, with $r_{1}+r_{2}=s+s^{*}+d+1$. We have 


$$
\begin{array}{rlr}
b_{0} & =\frac{-h d\left(1+r_{1}\right)\left(1+r_{2}\right)}{2+s^{*}}, \\
b_{i} & =\frac{h(i-d)\left(i+1+s^{*}\right)\left(i+1+r_{1}\right)\left(i+1+r_{2}\right)}{\left(2 i+1+s^{*}\right)\left(2 i+2+s^{*}\right)} & (1 \leq i \leq d-1), \\
c_{i} & =\frac{h i\left(i+d+1+s^{*}\right)\left(i+s^{*}-r_{1}\right)\left(i+s^{*}-r_{2}\right)}{\left(2 i+s^{*}\right)\left(2 i+1+s^{*}\right)} & (1 \leq i \leq d-1), \\
c_{d} & =\frac{h d\left(d+s^{*}-r_{1}\right)\left(d+s^{*}-r_{2}\right)}{2 d+s^{*}} .
\end{array}
$$

To get $\left\{b_{i}^{*}\right\}_{i=0}^{d-1}$ and $\left\{c_{i}^{*}\right\}_{i=1}^{d}$, in the above formulas exchange $h \leftrightarrow h^{*}, s \leftrightarrow s^{*}$ and preserve $r_{1}, r_{2}$.

Example 20.9 (Hahn). We have

$$
\begin{aligned}
\theta_{i} & =\theta_{0}+s i, \\
\theta_{i}^{*} & =\theta_{0}^{*}+h^{*} i\left(i+1+s^{*}\right)
\end{aligned}
$$

for $0 \leq i \leq d$ and

$$
\begin{aligned}
\varphi_{i} & =h^{*} s i(i-d-1)(i+r), \\
\phi_{i} & =-h^{*} s i(i-d-1)\left(i+s^{*}-r\right)
\end{aligned}
$$

for $1 \leq i \leq d$. We have

$$
\begin{aligned}
b_{0} & =\frac{-s d(1+r)}{2+s^{*}}, \\
b_{i} & =\frac{s(i-d)\left(i+1+s^{*}\right)(i+1+r)}{\left(2 i+1+s^{*}\right)\left(2 i+2+s^{*}\right)} \\
c_{i} & =\frac{-s i\left(i+d+1+s^{*}\right)\left(i+s^{*}-r\right)}{\left(2 i+s^{*}\right)\left(2 i+1+s^{*}\right)} \\
c_{d} & =\frac{-s d\left(d+s^{*}-r\right)}{2 d+s^{*}}
\end{aligned} \quad(1 \leq i \leq d-1),
$$

and

$$
\begin{array}{lc}
b_{i}^{*}=h^{*}(i-d)(i+1+r) & (0 \leq i \leq d-1), \\
c_{i}^{*}=h^{*} i\left(i-d-1-s^{*}+r\right) & (1 \leq i \leq d) .
\end{array}
$$

Example 20.10 (dual Hahn). We have 


$$
\begin{aligned}
\theta_{i} & =\theta_{0}+h i(i+1+s), \\
\theta_{i}^{*} & =\theta_{0}^{*}+s^{*} i
\end{aligned}
$$

for $0 \leq i \leq d$ and

$$
\begin{aligned}
& \varphi_{i}=h s^{*} i(i-d-1)(i+r), \\
& \phi_{i}=h s^{*} i(i-d-1)(i+r-s-d-1)
\end{aligned}
$$

for $1 \leq i \leq d$. We have

$$
\begin{array}{lc}
b_{i}=h(i-d)(i+1+r) & (0 \leq i \leq d-1), \\
c_{i}=h i(i-d-1-s+r) & (1 \leq i \leq d)
\end{array}
$$

and

$$
\begin{aligned}
& b_{0}^{*}=\frac{-s^{*} d(1+r)}{2+s}, \\
& b_{i}^{*}=\frac{s^{*}(i-d)(i+1+s)(i+1+r)}{(2 i+1+s)(2 i+2+s)} \quad(1 \leq i \leq d-1) \text {, } \\
& c_{i}^{*}=\frac{-s^{*} i(i+d+1+s)(i+s-r)}{(2 i+s)(2 i+1+s)} \quad(1 \leq i \leq d-1) \text {, } \\
& c_{d}^{*}=\frac{-s^{*} d(d+s-r)}{2 d+s} \text {. }
\end{aligned}
$$

Example 20.11 (Krawtchouk). We have

$$
\begin{aligned}
\theta_{i} & =\theta_{0}+s i \\
\theta_{i}^{*} & =\theta_{0}^{*}+s^{*} i
\end{aligned}
$$

for $0 \leq i \leq d$ and

$$
\begin{aligned}
\varphi_{i} & =r i(i-d-1) \\
\phi_{i} & =\left(r-s s^{*}\right) i(i-d-1)
\end{aligned}
$$

for $1 \leq i \leq d$. We have

$$
\begin{array}{lc}
b_{i}=r(i-d) / s^{*} & (0 \leq i \leq d-1), \\
c_{i}=i\left(r-s s^{*}\right) / s^{*} & (1 \leq i \leq d) .
\end{array}
$$

To get $\left\{b_{i}^{*}\right\}_{i=0}^{d-1}$ and $\left\{c_{i}^{*}\right\}_{i=1}^{d}$, in the above formulas exchange $s \leftrightarrow s^{*}$ and preserve $r$.

Example 20.12 (Bannai/Ito). We have 


$$
\begin{aligned}
\theta_{i} & =\theta_{0}+h\left(s-1+(1-s+2 i)(-1)^{i}\right), \\
\theta_{i}^{*} & =\theta_{0}^{*}+h^{*}\left(s^{*}-1+\left(1-s^{*}+2 i\right)(-1)^{i}\right)
\end{aligned}
$$

for $0 \leq i \leq d$ and

$$
\begin{aligned}
\varphi_{i}= & h h^{*}\left((-1)^{i} r_{2}-2 i-r_{2}\right)\left(2 i+r_{1}-d-1+(-1)^{i+d}\left(r_{1}+d+1\right)\right), \\
\phi_{i}= & h h^{*}\left(s^{*}+r_{2}+(-1)^{i}\left(2 i-s^{*}-r_{2}\right)\right) \\
& \times\left(d+1-s^{*}-r_{1}+(-1)^{i+d}\left(2 i-d-1-s^{*}-r_{1}\right)\right)
\end{aligned}
$$

for $1 \leq i \leq d$, with $r_{1}+r_{2}=-s-s^{*}+d+1$. Note that

$$
\begin{aligned}
\theta_{i} & =\left\{\begin{array}{cc}
\theta_{0}+2 h i, & \text { if } i \text { even; } \\
\theta_{0}+2 h(s-i-1), & \text { if } i \text { odd },
\end{array}\right. \\
\theta_{i}^{*} & =\left\{\begin{array}{cc}
\theta_{0}^{*}+2 h^{*} i, & \text { if } i \text { even; } \\
\theta_{0}+2 h^{*}\left(s^{*}-i-1\right), & \text { if } i \text { odd }
\end{array}\right.
\end{aligned}
$$

for $0 \leq i \leq d$ and

$$
\begin{aligned}
& \varphi_{i}=\left\{\begin{array}{cc}
-4 h h^{*} i\left(i+r_{1}\right), & \text { if } i \text { even, } d \text { even; } \\
-4 h h^{*}(i-d-1)\left(i+r_{2}\right), & \text { if } i \text { odd, } d \text { even; } \\
-4 h h^{*} i(i-d-1), & \text { if } i \text { even, } d \text { odd; } \\
-4 h h^{*}\left(i+r_{1}\right)\left(i+r_{2}\right), & \text { if } i \text { odd, } d \text { odd, }
\end{array}\right. \\
& \phi_{i}=\left\{\begin{array}{cc}
4 h h^{*} i\left(i-s^{*}-r_{1}\right), & \text { if } i \text { even, } d \text { even; } \\
4 h h^{*}(i-d-1)\left(i-s^{*}-r_{2}\right), & \text { if } i \text { odd, } d \text { even; } \\
-4 h h^{*} i(i-d-1), & \text { if } i \text { even, } d \text { odd; } \\
-4 h h^{*}\left(i-s^{*}-r_{1}\right)\left(i-s^{*}-r_{2}\right) & \text { if } i \text { odd, } d \text { odd }
\end{array}\right.
\end{aligned}
$$

for $1 \leq i \leq d$. We have

$$
\begin{aligned}
b_{i}= & h\left(2 i+2+r_{2}-s^{*}+(-1)^{i}\left(r_{2}+s^{*}\right)\right) \\
& \times \frac{2 i+r_{1}-d+1-(-1)^{i+d}\left(r_{1}+d+1\right)}{2\left(2 i+2-s^{*}\right)} \quad(0 \leq i \leq d-1), \\
c_{i}= & -h\left(2 i-r_{2}-s^{*}+(-1)^{i}\left(r_{2}+s^{*}\right)\right) \\
& \times \frac{2 i-2 s^{*}-r_{1}+d+1-(-1)^{i+d}\left(r_{1}+d+1\right)}{2\left(2 i-s^{*}\right)} \quad(1 \leq i \leq d) .
\end{aligned}
$$

Note that 


$$
b_{i}=\left\{\begin{array}{cc}
\frac{2 h(i-d)\left(i+1+r_{2}\right)}{2 i+2-s^{*}}, & \text { if } i \text { even, } d \text { even; } \\
\frac{2 h\left(i+1-s^{*}\right)\left(i+1+r_{1}\right)}{2 i+2-s^{*}}, & \text { if } i \text { odd, } d \text { even; } \\
\frac{2 h\left(i+1+r_{1}\right)\left(i+1+r_{2}\right)}{2 i+2-s^{*}}, & \text { if } i \text { even, } d \text { odd; } \\
\frac{2 h(i-d)\left(i+1-s^{*}\right)}{2 i+2-s^{*}}, & \text { if } i \text { odd, } d \text { odd }
\end{array}\right.
$$

for $0 \leq i \leq d-1$ and

$$
c_{i}=\left\{\begin{array}{cc}
\frac{-2 h i\left(i-s^{*}-r_{1}\right)}{2 i-s^{*}}, & \text { if } i \text { even, } d \text { even; } \\
\frac{-2 h\left(i+d+1-s^{*}\right)\left(i-s^{*}-r_{2}\right)}{2 i-s^{*}}, & \text { if } i \text { odd, } d \text { even; } \\
\frac{-2 h i\left(i+d+1-s^{*}\right)}{2 i-s^{*}}, & \text { if } i \text { even, } d \text { odd; } \\
\frac{-2 h\left(i-s^{*}-r_{1}\right)\left(i-s^{*}-r_{2}\right)}{2 i-s^{*}}, & \text { if } i \text { odd, } d \text { odd }
\end{array}\right.
$$

for $1 \leq i \leq d$.

To get $\left\{b_{i}^{*}\right\}_{i=0}^{d-1}$ and $\left\{c_{i}^{*}\right\}_{i=1}^{d}$, in the above formulas exchange $h \leftrightarrow h^{*}, s \leftrightarrow s^{*}$ and preserve $r_{1}, r_{2}, q$.

Example 20.13 (Orphan). Assume that $\operatorname{char}(\mathbb{F})=2$ and $d=3$. We have

$$
\begin{array}{lll}
\theta_{1}=\theta_{0}+h(1+s), & \theta_{2}=\theta_{0}+h, & \theta_{3}=\theta_{0}+h s, \\
\theta_{1}^{*}=\theta_{0}^{*}+h^{*}\left(1+s^{*}\right), & \theta_{2}^{*}=\theta_{0}^{*}+h^{*}, & \theta_{3}^{*}=\theta_{0}^{*}+h^{*} s^{*}
\end{array}
$$

and

$$
\begin{array}{lll}
\varphi_{1}=h h^{*} r, & \varphi_{2}=h h^{*}, & \varphi_{3}=h h^{*}\left(r+s+s^{*}\right), \\
\phi_{1}=h h^{*}\left(r+s+s s^{*}\right), & \phi_{2}=h h^{*}, & \phi_{3}=h h^{*}\left(r+s^{*}+s s^{*}\right) .
\end{array}
$$

We have

$$
\begin{aligned}
& b_{0}=\frac{h r}{1+s^{*}}, \quad b_{1}=\frac{h\left(1+s^{*}\right)}{s^{*}}, \quad b_{2}=\frac{h\left(r+s+s^{*}\right)}{1+s^{*}}, \\
& c_{1}=\frac{h\left(r+s+s s^{*}\right)}{1+s^{*}}, \quad c_{2}=\frac{h\left(1+s^{*}\right)}{s^{*}}, \quad c_{3}=\frac{h\left(r+s^{*}+s s^{*}\right)}{1+s^{*}} .
\end{aligned}
$$

To get $\left\{b_{i}^{*}\right\}_{i=0}^{2}$ and $\left\{c_{i}^{*}\right\}_{i=1}^{3}$, in the above formulas exchange $h \leftrightarrow h^{*}, s \leftrightarrow s^{*}$ and preserve $r$. 


\section{References}

1. Askey, R., Wilson, J.: A set of orthogonal polynomials that generalize the Racah coefficients or 6-j symbols. SIAM J. Math. Anal. 10, 1008-1016 (1979)

2. Bannai, E., Ito, T.: Algebraic Combinatorics I. Association Schemes. The Benjamin/Cummings Publishing Co. Inc., Menlo Park (1984)

3. Brouwer, A.E., Cohen, A., Neumaier, A.: Distance-Regular Graphs. Springer-Verlag, Berlin (1989)

4. Cerzo, D.R.: Structure of thin irreducible modules of a $Q$-polynomial distance-regular graph. Linear Algebra Appl. 433, 1573-1613 (2010)

5. Delsarte, P.: An algebraic approach to the association schemes of coding theory. Philips Research Reports Suppl. (1973)

6. Granovskii, Y.I., Lutzenko, I.M., Zhedanov, A.S.: Mutual integrability, quadratic algebras, and dynamical symmetry. Ann. Phys. 217, 1-20 (1992)

7. Hanson, E.: A characterization of bipartite Leonard pairs using the notion of a tail. Linear Algebra Appl. 452, 46-67 (2014)

8. Helgason, S.: Differential Geometry and Symmetric Spaces. Academic Press, New York (1962)

9. Ito, T., Terwilliger, P.: Tridiagonal pairs of $q$-Racah type. J. Algebra 322, 68-93 (2009)

10. Koekoek, R., Lesky, P.A., Swarttouw, R.: Hypergeometric Orthogonal Polynomials and their $q$ Analogues. With a foreword by Tom H. Koornwinder. Springer Monographs in Mathematics. Springer-Verlag, Berlin (2010)

11. Labelle, J.: Tableau d'Askey. Orthogonal polynomials and applications (Bar-le-Duc, 1984), xxxvixxxvii, Lecture Notes in Math., 1171, Springer, Berlin (1985)

12. Leonard, D.A.: Orthogonal polynomials, duality and association schemes. SIAM J. Math. Anal. 13, 656-663 (1982)

13. Martin, W.J., Tanaka, H.: Commutative association schemes. Eur. J. Combin. 30, 1497-1525 (2009)

14. Terwilliger, P.: Leonard pairs and dual polynomial sequences (1987). https://www.math.wisc.edu/ $\sim$ terwilli/lphistory.html

15. Terwilliger, P.: The subconstituent algebra of an association scheme. I. J. Algebraic Combin. 1, 363-388 (1992)

16. Terwilliger, P.: The subconstituent algebra of an association scheme. III. J. Algebraic Combin. 2, 177-210 (1993)

17. Terwilliger, P.: Two relations that generalize the $q$-Serre relations and the Dolan-Grady relations. In: Physics and Combinatorics 1999 (Nagoya), pp. 377-398. World Scientific Publishing, River Edge (2001)

18. Terwilliger, P.: Two linear transformations each tridiagonal with respect to an eigenbasis of the other. Linear Algebra Appl. 330, 149-203 (2001)

19. Terwilliger, P.: Two linear transformations each tridiagonal with respect to an eigenbasis of the other; the TD-D canonical form and the LB-UB canonical form. J. Algebra 291, 1-45 (2005)

20. Terwilliger, P.: Leonard pairs and the $q$-Racah polynomials. Linear Algebra Appl. 387, 235-276 (2004)

21. Terwilliger, P.: Two linear transformations each tridiagonal with respect to an eigenbasis of the other; comments on the parameter array. Des. Codes Cryptogr. 34, 307-332 (2005)

22. Terwilliger, P.: Two linear transformations each tridiagonal with respect to an eigenbasis of the other: comments on the split decomposition. J. Comput. Appl. Math. 178, 437-452 (2005)

23. Terwilliger, P.: An Algebraic Approach to the Askey Scheme of Orthogonal Polynomials. Orthogonal Polynomials and Special Functions, Lecture Notes in Math, pp. 255-330. Springer, Berlin (2006)

24. Tits, J.: Sur certaines classes d'espaces homogenes de groupes de Lie. Mem. Acad. Roy. Belg. Cl. Sci. 3, 268 (1955)

25. Wang, H.C.: Two-point homogeneous spaces. Ann. Math. 55, 177-191 (1952)

26. Zhedanov, A.: Hidden symmetry of Askey-Wilson polynomials. Theor. Math. Phys. 89, 1146-1157 (1991)

Publisher's Note Springer Nature remains neutral with regard to jurisdictional claims in published maps and institutional affiliations. 